University of Wisconsin Milwaukee

UWM Digital Commons

Theses and Dissertations

May 2014

\title{
Testing Components of a Self-management Theory in Adolescents with Type 1 Diabetes Mellitus
}

Gwen Marie Verchota

University of Wisconsin-Milwaukee

Follow this and additional works at: https://dc.uwm.edu/etd

Part of the Nursing Commons

\section{Recommended Citation}

Verchota, Gwen Marie, "Testing Components of a Self-management Theory in Adolescents with Type 1 Diabetes Mellitus" (2014).

Theses and Dissertations. 776.

https://dc.uwm.edu/etd/776

This Dissertation is brought to you for free and open access by UWM Digital Commons. It has been accepted for inclusion in Theses and Dissertations by an authorized administrator of UWM Digital Commons. For more information, please contact open-access@uwm.edu. 
TESTING COMPONENTS OF A SELF-MANAGEMENT THEORY IN ADOLESCENTS WITH TYPE 1 DIABETES MELLITUS

by

\author{
Gwen M. Verchota \\ A Dissertation Submitted in \\ Partial Fulfillment of the \\ Requirements for the Degree of \\ Doctor of Philosophy \\ in Nursing
}

at

The University of Wisconsin-Milwaukee

May, 2014 


\title{
ABSTRACT \\ TESTING COMPONENTS OF A SELF-MANAGEMENT THEORY IN ADOLESCENTS WITH TYPE 1 DIABETES MELLITUS
}

by

Gwen Verchota

The University of Wisconsin-Milwaukee, 2014

Under the Supervision of Dr. Kathleen Sawin

\begin{abstract}
Advances in treatment technology and the importance of obtaining normoglycemia in order to prevent or delay complications associated with Type 1 Diabetes Mellitus (T1DM) has shifted much of the emphasis of diabetes self-management (SM) onto the adolescent and his or her family. The primary responsibility for managing T1DM in childhood is with the parent whereas during adolescence, increasing levels of responsibility for SM are transferred to the adolescent. This study examined the relationships of key context and process variables on proximal (self-management behaviors $[\mathrm{SMB}]$ ) and distal outcomes (metabolic control and diabetes-specific healthrelated quality of life [DQOL]) from the Individual and Family Self-management Theory (IFSMT) in a cohort of adolescents with T1DM. The aims in this correlational, crosssectional study included testing components of the IFMST by examining relationships among select context, process, and outcome variables thought to contribute to effective SMB in adolescents with T1DM and determining whether SMB mediated the relationship


of IFSMT process variables on metabolic control and DQOL. Families were recruited from an ambulatory diabetes clinic in a Midwestern children's hospital. Participants included 103 adolescent-parent dyads (adolescents aged 12-17 years with T1DM) from an outpatient diabetes clinic. The dyads each completed a self-report survey including instruments intended to measure study variables from the IFSMT. Using hierarchical multiple regression, context (depressive symptoms) and process (communication) variables explained $37 \%$ of the variance in SMB. Regimen complexity and depressive symptoms explained $11 \%$ of the variance in metabolic control. Only regimen complexity was significant at Step 1. Neither of the regression steps adding process variables or SMB were significant. For DQOL, context (depressive symptoms) variables explained $26 \%$ of the variance at Step 1, 36\% of the variance when process (self-efficacy and communication) variables were added at Step 2 and 52\% of the total variance was explained when SM behaviors were added at Step 3. In the final model, three variables were significant: depressive symptoms, self-efficacy, and SMB. The criteria for mediation of SMB by the process variables were not met. IFSMT served as a cogent model for understanding key concepts, processes, and outcomes essential to SM in adolescents and families dealing with the complex disease, T1DM. 
(C) Copyright by Gwen Verchota, 2014

All Rights Reserved 


\section{DEDICATION}

While still in my 20's I accepted a position as "Diabetes Nurse Clinician" where I was charged with developing a regional ambulatory and in-patient diabetes program for individuals and families dealing with a diagnosis of gestational, Type 1, or Type 2 Diabetes Mellitus. Every August for 12 consecutive years (except for the year I got married) I took a group of 10-12 adolescents on a week-long canoe trip. It was on these trips that I learned first-hand how diabetes impacts the lives of adolescents and how difficult it is to achieve 'good' metabolic control— even in a natural outdoor 'laboratory' where I could observe dietary intake and activity levels, validate blood sugars, review basal and bolus insulin doses as they were carefully programmed into pumps, help draw up insulin and watch as shots were self-administered based on 'best evidence' algorithms. I still have vivid memories of checking every camper's blood sugar at 3:00 a.m. while camping on the banks of the Namakagon River because no one had a blood sugar over 60 $\mathrm{mg} / \mathrm{dL}$ at the bedtime check, despite following meticulously calculated algorithms!

I dedicate this dissertation to all the courageous adolescents with T1DM that have impacted my practice, career, and life; adolescents from summer camping adventures; adolescents and families who trusted me to partner with them as their primary care provider; and the adolescent and parent dyads who willingly agreed to participate in this study. I am grateful to them for helping us better understand a significant part of their everyday reality. 


\section{TABLE OF CONTENTS}

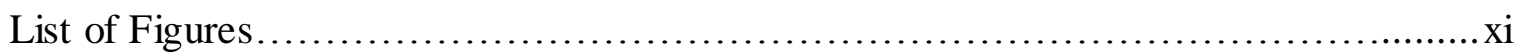

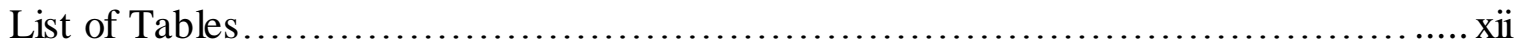

List of Appendices............................................................... xii

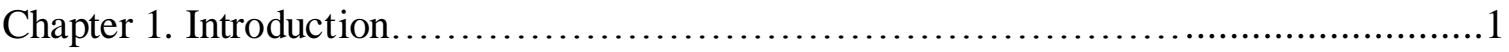

Incidence and Prevalence of Type 1 Diabetes in Adolescents................................1

The Impetus for Tight Metabolic Control.................................................

Diabetes Management Responsibilities......................................................

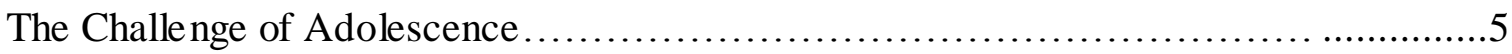

Individual and Family Self-Management Theory...................................6

The Historical Evolution of the Concept of Self-Management.............................8

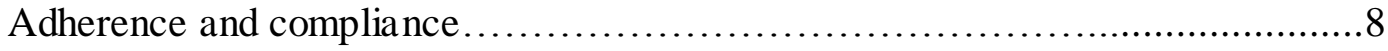

Self-care and self-care autonomy................................................... 10

Shortcomings associated with adherence, compliance and self-care...................12

Conceptualization and Operationalization of Adolescent Self-management.........13

Gaps in our Understanding of Self-management Behaviors and Health Outcomes........15

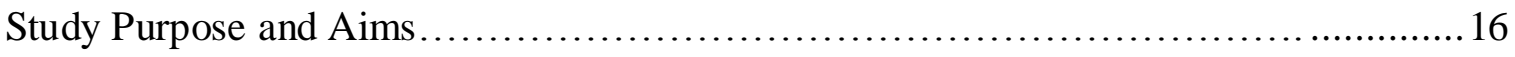

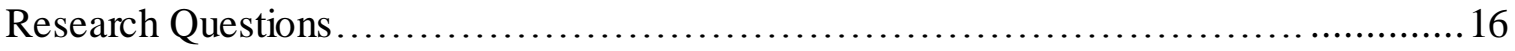

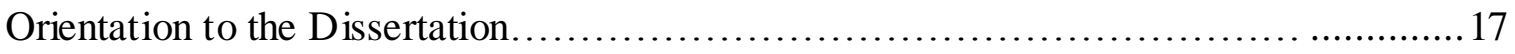

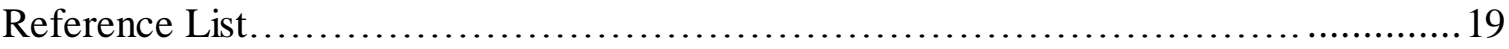

Chapter 2. Optimizing Self-manage ment in Adolescents with T1DM..................25 
Abstract. .25

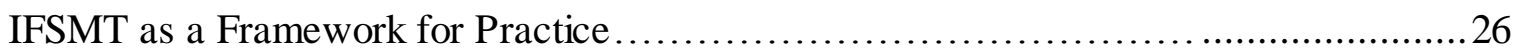

Context Factors and the ir Relationship to Self-Management Behaviors..........28

Condition-specific contextual factors ......................................28

Physical and social environment......................................... 30

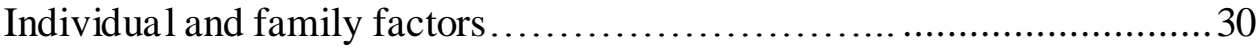

Process Factors and their Relationship to Self-Management Behaviors.............33

Know ledge and beliefs............................................ 33

Self-regulation skills and abilities................................... 34

Social facilitation.............................................. 35

Process Related Interventions.......................................... 37

Outcomes.......................................................... 38

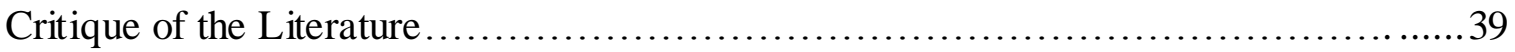

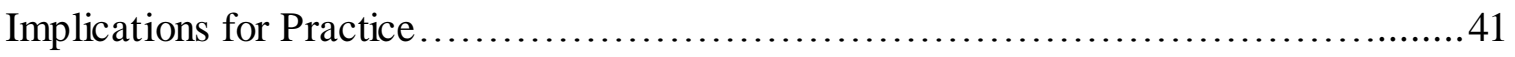

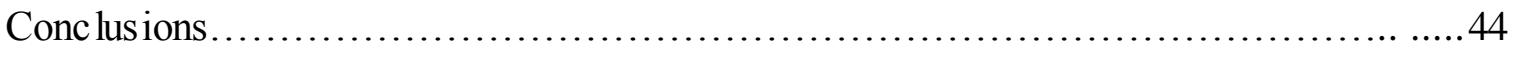

Reference List................................................................ 45

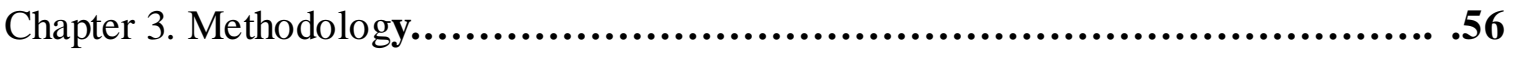

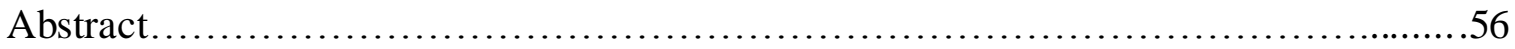

Evaluating Missing Data................................................... 57

Assessing Quantity and Patterns of Missing Data..............................57

Class ifying Missing Data............................................. 58

Missing value mechanis ms .............................................5 59 
Ignorability. .61

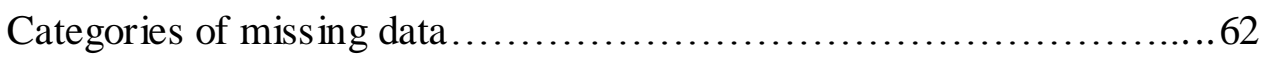

The Gap Between Recommendations and Reality ...........................6 63

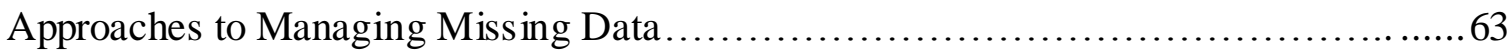

Traditional Missing Data Techniques......................................6 63

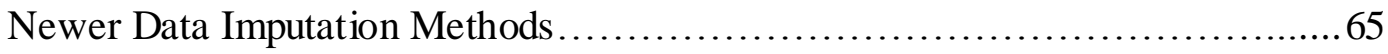

Multiple imputation (MI) ......................................... 65

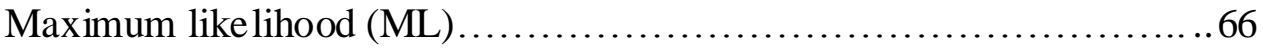

Practical Steps for Handling Missing Data in Survey Research Design...................67

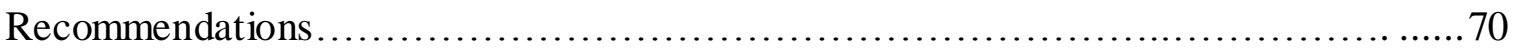

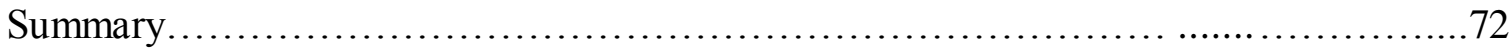

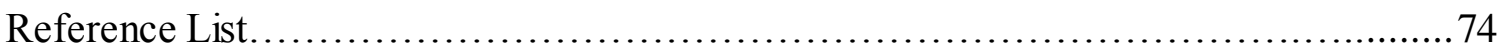

Chapter 4. Testing Components of a Self-manage ment Theory.........................77

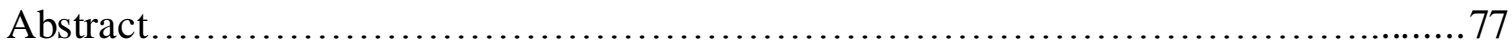

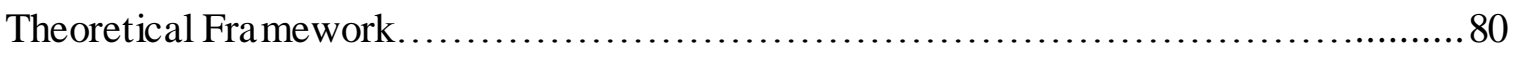

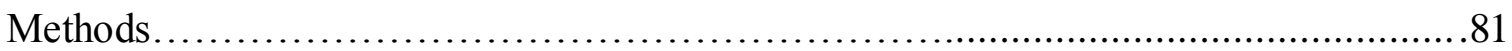

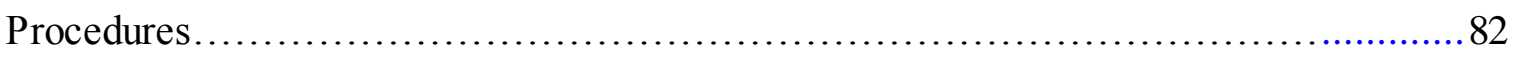

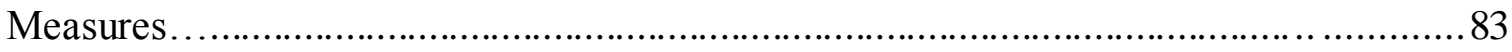

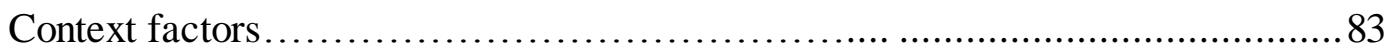

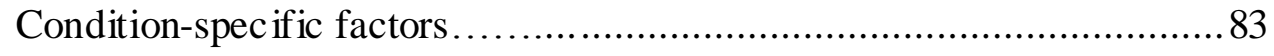

Individual \& Family Factors............................................................ 84

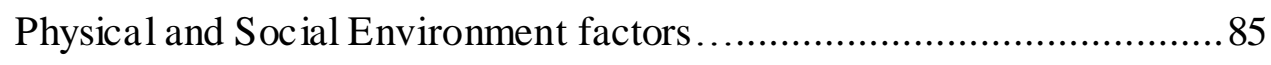




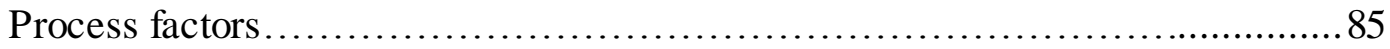

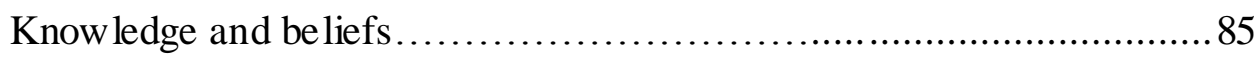

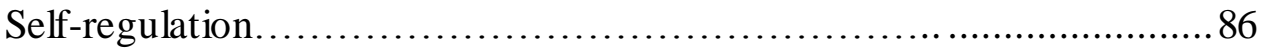

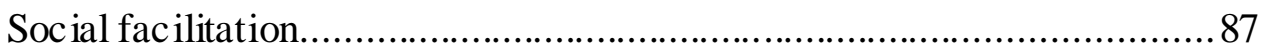

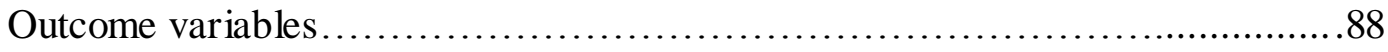

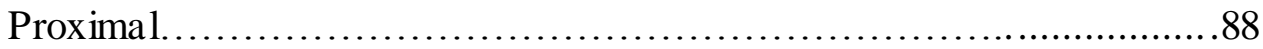

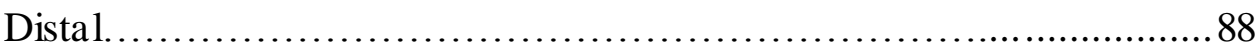

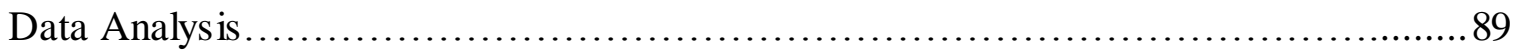

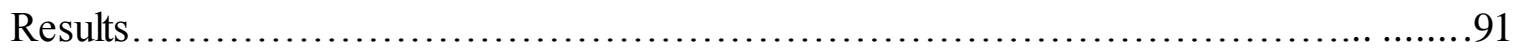

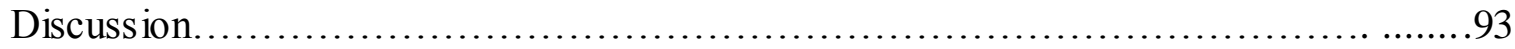

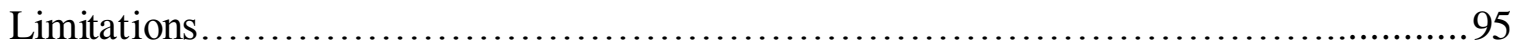

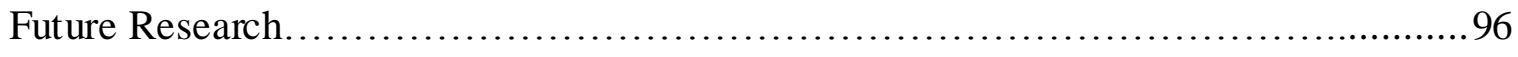

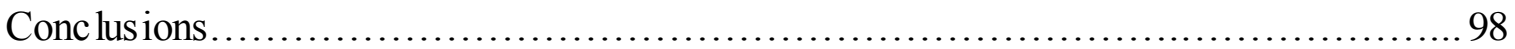

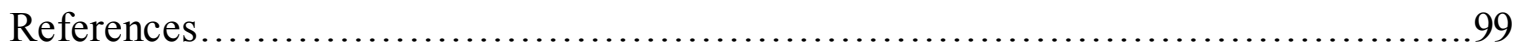

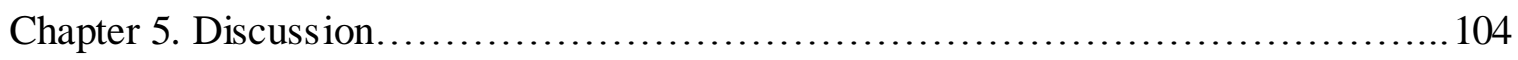

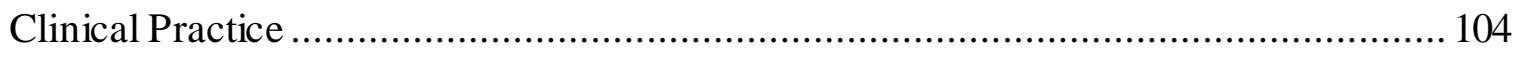

Measuring Se lf-Ma nagement Variables ......................................................... 104

Building Blocks to Diabetes SM: Self-regulation and Abilities ........................ 106

Building Blocks to Diabetes Self-Management: Social Facilitation .................. 109

Variables Impacting Metabolic Control........................................................... 110

Variables Impacting Diabetes Related Quality of Life ..................................... 112

Nurses Role in Caring for Adolescents with T1DM ................................................. 112 


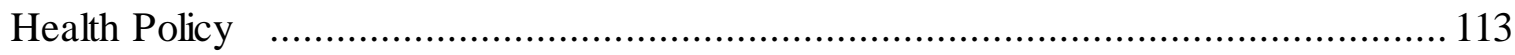

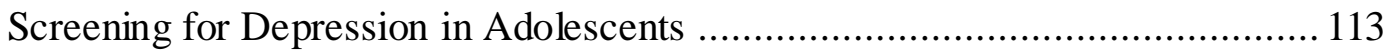

Barriers Associated with Mental Health Screening ......................................... 114

Depression in Adolescents with Type 1 Diabetes Mellitus .............................. 117

The Relationships between the States, Medicaid, and Mental Health ............................ 118

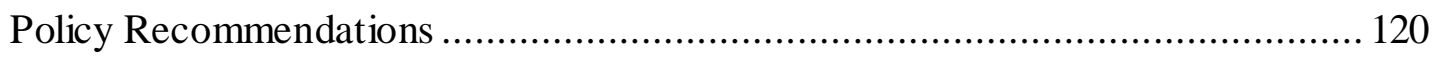

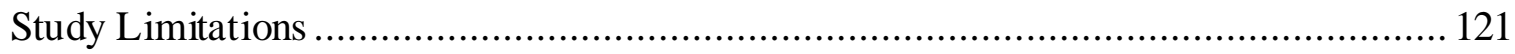

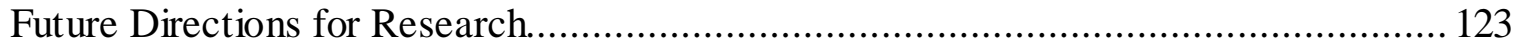

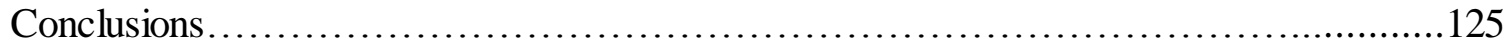

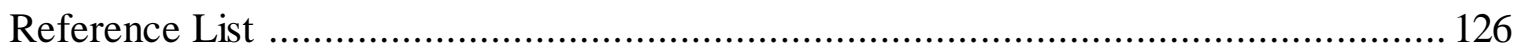

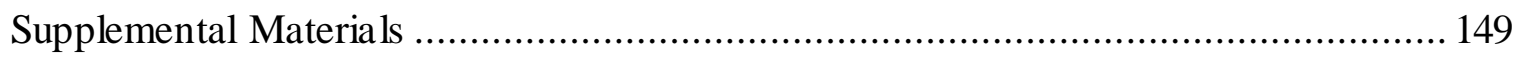




\section{LIST OF FIGURES}

\section{FIGURE}

1. Model of the Individual and Family Self-Management Theory..............132

2. Process-related intervention studies demonstrating greater efficacy......133

3. Application of the Individual and Family Self-Management Theory to Care of the Adolescents with T1DM...................................134

4. Syntax from SPSS, Missing Values Analysis, Expectation Maximization example.......................................................135 


\section{LIST OF TABLES}

\section{TABLE}

1. Intervention studies addressing the process dimension of SM.............136

2. SMODA-A Goals Subscale; EM Coefficients and Little's MCAR

Findings from SPSS Output................................. 142

3. Comparison of Listwise Deletion, Mean Substitution, and EM

Approach using Missing Data from the PedsQL

4. Comparison of Listwise, Deletion, Mean Substitution, and

Expectation Maximization Approaches for Self-Management

Activities, Goals, Communication and Depressive Symptoms

Instruments...........................................................

5. Context Variables..................................................... 144

6. Process and Outcome Variables......................................145

7. Correlations between Context, Process, and Outcome Variables.........146

8. Hierarchical Multiple Regression Analysis..........................147 


\section{LIST OF APPENDICES}

Appendix A: Children's IRB Approval.............................. 148

Appendix B: IRB Consent/Assent Form..........................149

Appendix C: Child Assent Form................................. 155

Appendix D: Pediatric Endocrinology Section Support for Adolescent

Recruitment................................................ 158

Appendix E: Demographics and Clinical Information Form..............160

Appendix F: Curriculum Vita................................. 162 


\section{ACKNOWLEDGEMENTS}

I would like to acknowledge several of the many individuals who helped along the way to make completing the $\mathrm{PhD}$ a reality for me. To my husband, Bob, and children, Katie and Bobby, who became increasingly independent and capable of many things they didn't think they could do as the dissertation work continued on. I would like to thank them for their steadfast support, love and belief that I could make it to the finish line.

The following foundations provided funding for various components of the study: Harriet H. Werley Doctoral Student Research Scholarship, Southeastern Wisconsin Research Consortium Scholarship, and the 2010 Sigma Theta Tau International, Theta Xi Chapter Doctoral Student Research Scholarship. These resources were greatly appreciated.

Dr. Sawin served as an exemplary mentor; the quintessence of a combined nurse scientist, theorist, faculty, and scholar. Her tireless spirit, challenging observations, and words of wisdom helped further support and enhance my learning and understanding of theory development, research, chronic disease management, and practice. I will be forever grateful for our four years of study together. I would also like to acknowledge Dr. Weiming Ke, a statistics consultant and faculty who co-authored the Missing Data manuscript with me.

I would like to thank members of my Committee who willingly accepted the invitation to join me in this doctoral journey and demonstrate once again, how bringing together a group of colleagues from across health care disciplines can enhance outcomes. 
Committee members included Dr. Jessica Kichler, Dr. Joshua Mersky, Dr. Rachel

Schiffman, and Dr. Julia Snethen. Their participation, ideas shared during the journey, and thoughtful critique were invaluable and I am grateful to each one of them for their contributions. 


\section{Chapter 1}

Introduction

\section{Incidence and Prevalence of Type 1 Diabetes in Adolescents}

Each year, more than 15,000 youth are diagnosed with Type 1 Diabetes Mellitus (T1DM) in the United States (Juvenile Diabetes Research Foundation [JDRF], 2009). This equates to 40 children and adolescents diagnosed daily. About 186,300 people younger than 20 years of age have diabetes in the United States (JDRF, 2009). Recent epidemiological data indicate the most common age of onset of T1DM is from 10 to 14 years, with the incidence of diabetes increasing worldwide (Karvonen et al., 2000).

\section{The Impetus for Tight Metabolic Control}

A commonly held assumption is that individuals with T1DM are at increased risk for developing chronic complications including retinopathy, renal disease, and neuropathy if diabetes is not well controlled. In the landmark Diabetes Control and Complications Trial (DCCT, 1993) a clear, decreasing risk for the onset and progression of complications in relation to improved metabolic control was demonstrated. The DCCT enrolled 1,441 individuals with T1DM and randomized them into either an intensive or conventional treatment group. Participants in the intensive arm administered insulin three or more times a day by either injection (long-acting insulin along with multiple doses of rapid-acting insulin given prior to meals) or by maintaining a continuous subcutaneous insulin infusion (CSII) via an insulin infusion pump. Goals for the intensively managed subjects included blood glucoses 70-120 milligrams per deciliter 
(mg/dL) on pre-meal monitoring; post-prandial glucoses less than $180 \mathrm{mg} / \mathrm{dL}$, and a weekly 3 a.m. reading greater than $65 \mathrm{mg} / \mathrm{dL}$. Adjustments were made to the insulin regimen based on the results of frequent blood glucose monitoring (four or more times a day), exercise/physical activity, and dietary intake. Hemoglobin A1c (HgbA1c) was measured monthly with a goal of establishing normoglycemia (HgbA1c less than 6.05\%). Participants from the intensively managed cohort were seen in the clinic on a monthly basis and were contacted between visits by telephone to review and adjust their treatment regimens. The conventional group treatment, i.e., treatment as usual, consisted of one or two daily insulin injections (mixed intermediate and rapid acting insulin) and daily selfblood glucose or urine monitoring. These subjects received diet and exercise education and in this cohort, insulin dose adjustments were not encouraged. HgbA1c was measured quarterly, but neither the participant nor the health care team was aware of the result unless the value was greater than $13.1 \%$. Participants from the conventional cohort were seen in the clinic at 3-month intervals.

A follow-up report focusing exclusively on the adolescent cohort of the DCCT (1994) further established that intensive treatment with CSII or multiple daily injections (MDI) along with frequent self-blood glucose monitoring benefited this subset of adolescents by preventing or delaying the onset of chronic complications. Participants included 195 adolescents and young adults who were between 13 and 17 years of age when they enrolled in the DCCT. The mean duration of time spent in the study for subjects was 7.4 years (range: 4 to 9 years). The DCCT (1994) findings demonstrated that by consistently lowering plasma glucose levels in the adolescent cohort, the risk of 
diabetes-related complications decreased by 53-70\%, placing yet further emphasis on the importance of maintaining optimal metabolic control during adolescence and beyond (DCCT). A downside to the lower plasma glucose levels was the risk of weight gain and hypoglycemia: Severe hypoglycemia was two to four times greater in intensively treated subjects than in the conventional treatment group (DCCT).

After the DCCT study officially ended, all subjects were invited to participate in the Epidemiology of Diabetes Interventions and Complications (EDIC) trial (White et al., 2001). The EDIC was a 4- year study designed to determine whether the benefits of improved metabolic control persisted over time. Participants from the original DCCT conventional treatment group were provided with education and assistance in following intensive treatment principles and modalities and the intensive treatment group was encouraged to continue strict management of their diabetes. One hundred seventy-five of the original 195 adolescent subjects continued in the EDIC study. HgbA1c levels during the EDIC study were comparable between the former intensive and conventionally treated groups, yet the prevalence of worsening retinopathy was reduced by $74 \%$ in adolescents who had been intensively managed in the DCCT study. This subset from the cohort of adolescents in the study was also $78 \%$ less likely to progress to proliferative or severe nonproliferative retinopathy than adolescents from the prior conventionally managed cohort (White et al.). These two seminal works, the DCCT and EDIC demonstrated what had been speculated in adolescents with T1DM: Improved metabolic control minimized chronic complications associated with T1DM. 


\section{Diabetes Management Responsibilities}

Research findings suggest that parents influence how seriously diabetes will affect youth with T1DM because they are involved in the details of managing diabetes in the early years and in preparing older children/adolescents for the role of self-managing diabetes and incorporating life-long health habits into their lives (Hanna \& Guthrie, 2003; King, Berg, Butner, Butler \& Wiebe, 2013). It is not uncommon for parents to feel vulnerable, especially early in the disease process, as they learn that their newly diagnosed child or adolescent can potentially develop acute life-threatening crises and serious chronic complications if they do not learn to manage diabetes effectively. Parents soon recognize that being able to successfully manage T1DM means learning how to incorporate a rigorous diabetes regimen into the daily life of the family.

Ellis, Podolski, Frey, Naar-King, Wang \& Moltz (2007) suggested that parental support and monitoring of adolescent self-management behaviors impacted health outcomes including completion of self-management activities and metabolic control. Leonard, Garwick, and Adwan (2005) studied adolescent's (age 14-16) perceptions of family roles and relationships and parental involvement in managing T1DM and found perception of family functioning and parental support correlated with metabolic control and following the treatment regimen. Adolescents in the study acknowledged that even though their parents continued to be involved in monitoring diabetes management, the involvement was less frequent and less intense than when they were younger. Leonard et al. asserted that the parent-adolescent relationship can serve as a "strong" protective factor in relation to diabetes management when the adolescent views the parent as 
supportive and the relationship between parent and adolescent "emotionally close." Leonard et al. asserted that adolescents who described a relationship of conflict (in relation to diabetes management) between parent(s) and adolescent were at greater risk for poorer metabolic control.

\section{The Challenge of Adolescence}

Diabetes has been described as one of the most behaviorally and psychologically demanding chronic diseases due to the extensive daily requirements associated with selfmanagement (SM). The developmental period of adolescence adds a particularly challenging dimension for youth with diabetes. Research consistently demonstrates that children with diabetes experience a decline in diabetes self-management and metabolic control as they enter adolescence and poor metabolic control often persists throughout adolescence (Skinner \& Hampson, 2001; Chien et al., 2007).

Two significant factors implicated in preventing adolescents from maintaining good metabolic control include difficulty keeping up with increasing insulin requirements caused by hormonal changes associated with puberty and findings that adolescents' perform poorer self-care behavior compared to adults and younger children (Goran \& Gower, 2001; Moran, Jacobs, Steinberger, Luepker, \& Sinaiko, 1999). Amiel, Sherwin, Simonson, Lauritano, \& Tamborlane, (1986) found adolescents with and without T1DM were more insulin-resistant than adults, thus further contributing to the difficulties adolescents experience in achieving optimal metabolic control. Therefore, it comes as no surprise that adolescents have more diabetes management problems than either schoolage children or adults (Anderson. 2009). 
It was hypothesized that Individual and Family Self-management Theory (IFSMT) could serve as a guiding framework for nurses to use when caring for adolescents with T1DM and their families and further understand significant factors that impact health outcomes associated with T1DM in adolescence. The following section introduces the IFSMT.

\section{Individual and Family Self-Management Theory}

Individual and Family Self-Management Theory (IFSMT) proposes that successful management of T1DM during adolescence requires active participant involvement by the individual with T1DM coupled with additional support from family members who share responsibility for disease management.

IFSMT conceptualizes SM as:

A process in which individuals and families use knowledge and beliefs, selfregulation skills and abilities, and social facilitation to achieve health-related outcomes. Self-management takes place in the context of risk and protective factors specific to a health condition, physical and social environment and individual and family. Self-management is applicable to chronic conditions as well as health promotion" (UWM SMSC, 2011; Ryan and Sawin; 2009, Ryan 2009).

Ryan and Sawin assert that researchers have traditionally studied SM either from an individual lens or from a family lens but have not viewed individuals and families collectively. They maintain that using both lenses concurrently allows for a more comprehensive perspective and an ability to detect the changing dynamics within an individual and family system. The family unit in this model is not limited to biological families alone. 
IFSMT encompasses three broad dimensions; context, process, and outcome (Figure 1). The context dimension includes condition-specific risk and protective factors, the physical and social environment, and characteristics of individuals and family members" (Ryan \& Sawin, 2009). Ryan and Sawin theorized that contextual factors have the ability to directly impact individual and family engagement in the process of SM and the outcomes associated with SMB. The second dimension is the process dimension, which includes knowledge and beliefs, self-regulation skills and abilities, and social facilitation. Ryan and Sawin postulated that by enhancing the process of SM, proximal and distal outcomes associated with T1DM will improve for individuals and families.

Outcomes in IFSMT can be either proximal or distal. Proximal outcomes are specific behaviors an individual and family undertake to manage a condition, disease risk, symptoms, and/or drug therapies (Ryan \& Sawin, 2009). Achieving proximal outcomes impact success of distal outcomes. Distal outcomes include three unique categories: health related quality of life, health status, and costs associated with health. Obtaining optimal diabetes self-management behaviors (SMB) (a proximal outcome) affects HRQOL and metabolic control (distal outcomes) as measured by HgbA1c and diabetesspecific quality of life (DQOL) and costs associated with adolescent and family health. From the lens of IFSMT, health related behaviors include an individual's or family's daily activities (Ryan \& Sawin, 2009). An individual's capacity and needs affect how the individual and family are able to manage a chronic condition (Ryan \& Sawin, 2009). The sharing of responsibilities that occurs between parent and adolescent is referred to as dynamic and fluid shifting that occurs as the individual and family 
attempt to balance various roles and responsibilities essential to managing T1DM. Each component of the IFSMT will be described more fully in Chapter 2.

\section{The Historical Evolution of the Concept of Self-Management}

The term diabetes self-management $(\mathrm{SM})$ is a term that is often used in the diabetes literature referencing self-adjustment of insulin dosages or the daily tasks associated with following prescribed treatment recommendations. In much of the early diabetes literature, SM referred to the mastery of technical skills, like blood glucose monitoring. More recently, SM evolved into a term used to describe the shared responsibility assumed by individuals and families with diabetes (Schilling, Grey \& Knafl, 2002). To further complicate this issue, SM is often used interchangeably throughout the diabetes literature with such terms as adherence, self-care, and compliance (Goodall \& Halford, 1991; Schilling et al., 2002). A short review of these concepts is included to provide background to support the transition to SM as the preferred concept.

\section{Adhe rence and compliance}

Adherence refers to how well an individual's behavior coincides with a treatment plan prescribed by a health care provider. In the literature, adherence and non-adherence are typically viewed from one of two perspectives. One perspective involves categorizing patients as either adherent or non-adherent by some arbitrary means, without regard to theoretical or operational grounding. The other perspective considers adherence in relation to behavioral components of diabetes management, addressing how an individual's behavior(s) compares with a health care provider's set of prescriptive directives developed for them. 
Adherence is often used interchangeably with the term compliance in the diabetes literature. DiMatteo (2004) defined adherence as patient "compliance or acceptance and follow-through with treatment recommendations" (p. 207). La Greca (1990) defined treatment adherence as following without modification. Burroughs, Pontius, and Santiago (1993) studied health care compliance by measuring adherence to a diabetes regimen and the frequency by which certain behaviors occurred. Auslander, Thompson, Dreitzer, White, and Santiago (1997) defined adherence as the extent to which a patient follow recommended medical advice. Pereira, Berg-Cross, Almeida, and Machado (2008) studied adherence to diabetes treatment and suggested there are two types of adherence: behavioral adherence addressing meals, exercise, and frequency of insulin administration and medical adherence encompassing insulin administration and SBGM. Hoffman's (2002) definition was closer to SM as espoused by the IFSMT. He suggested that adherence "implies that the patient plays an active role rather than just bending to the physician's or care team's directions" (p. 129). Hoffman also linked this individualcentered adherence to treatment outcomes. The goal, argued Hoffman, was to prevent nonadherence because it is a primary contributor to poor diabetes control.

One of the few references relating the concept of adherence to any kind of theoretical underpinning was found in an article by Streisand and Mednick (2006) where adherence was grounded in self-care and Orem's theoretical framework. Streisand and Mednick described adherence as the "behaviors an individual with diabetes performs on his/her own behalf, to maintain life, health, and well-being" (p. 190). 
Hanson, Henggeler, and Burghen (1987) developed a measure of adherence behaviors based on data obtained from the American Diabetes Association and research on adherence behaviors. As a result, the Self-Care Adherence Inventory (SCAI) was born. Five types of what were referred to as adherence behaviors at the time but are clearly SMB (diet, insulin adjustment, SBGM, hypoglycemia management, and foot care) were combined into a single global measure. Harris et al. (2000) updated the content of the SCAI and changed the title to the Diabetes Self-Management Profile (DSMP). The DSMP is purported to evaluate "behaviors" in adolescents with T1DM including insulin administration and dose adjustment, SBGM, exercise, diet, and hypoglycemia management. The DSMP has strong internal consistency, inter-rater and retest reliability, and construct validity (Harris, 2000; Iannotti et al., 2006). Lewin et al. (2010) added normative data to the DSMP allowing for age and gender comparisons. A shortcoming of the DSMP is that it requires detailed training of interviewers, has lower reliability subscale scores when compared to total scores and has been described as burdensome for participants to complete (Wysocki, Buckloh, Antal, Lochrie, \& Taylor, 2012).

\section{Self-care and self-care autonomy}

Many researchers have studied the concept of self-care and the relationship between the distribution of responsibility for diabetes self-care and diabetes outcomes within families. During childhood, the primary responsibility for managing diabetes lies with the parent whereas during adolescence, increasing levels of responsibility for diabetes care are transferred to the adolescent as he or she matures (Anderson, Ho, Brackett, Finkelstein \& Laffel, 1997; King et al., 2013). It has been suggested that there 
is often an increase in conflict between youth with diabetes and their parents, stemming from how much the youth is participating in self-care and determining who is responsible for disease management (Anderson et al., 2002; Urban \& Grey, 2006). Several researchers demonstrated that greater parental involvement is associated with improved self-care behavior on the part of the adolescent (Anderson et al., 1997; King et al., 2013; Leonard et al., 2005) and ultimately improved diabetes control (Leonard et al., 2005). Conversely, other researchers failed to demonstrate a relationship between parental responsibility and improved self-care behaviors in adolescents (Miller \& Drotar, 2003; Weibe et al., 2005) or improved metabolic control (Anderson et al., 1997; Miller \& Drotar, 2003; Weibe et al., 2005).

A number of research studies addressed diabetes responsibility sharing between parent and child. Wysocki et al. (1996) examined self-care autonomy and psychological maturity in youth ages five to 17 years with T1DM. Self-care autonomy consisted of a measure of parent-child sharing of diabetes responsibilities and the parents' perception of their child's diabetes SM capabilities. They discovered that excessive self-care autonomy, described as limited or no parental supervision, was associated with lower levels of treatment adherence and knowledge, higher hospitalization rates, and poorer metabolic control than youth with appropriate or "constrained" self-care autonomy. La Greca et al. (1990) found that preadolescents who assumed a high level of responsibility for diabetes care displayed poorer metabolic control than those who assumed less responsibility but whose parents were more involved in daily management. La Greca et al. cautioned that too much self-care autonomy could hinder optimal metabolic control. 
Grey, Davidson, Boland, and Tamborlane (2001) conducted a longitudinal intervention study and found youth age 12-20 (mean age: $14.3 \pm 2 S D$ ) whose parents maintained involvement in their diabetes treatment had better overall metabolic control than those who did not.

\section{Shortcomings ass ociated with adhe rence, compliance and se If-care}

Adherence, compliance, self-care and self-care autonomy are common constructs in the diabetes behavioral literature. Ryan and Sawin (2009) assert that the concept of adherence is contrary to SM because adherence does not support the idea that the primary responsibility and control necessary for SM to occur lies with the individual and family. Consistent with the theory, the adolescent with T1DM and his or her family are fully responsible for the multidimensional nature of SM within a structure of support and collaboration with health care resources. Ryan and Sawin maintained that self-care is associated with but separate from SM; self-care encompasses performing activities of daily living but once again does not include the larger role of controlling and managing the condition through a collaborative process with health care professionals.

A majority of research studies have focused on the study of relationships between what was historically called adherence but were often SMB, and clinical outcomes, namely metabolic control in adolescents with T1DM. With the volume of research that has accrued with a primary focus on these SMB (often labeled as adherence) in youth with T1DM, we remain without answers as to how these behaviors and similar constructs change, how to effect changes in these behaviors, or how support from family, health care providers, or others impact these constructs. 


\section{Conceptualization and ope rationalization of adoles cent self-management}

A concept analysis addressing SM of T1DM during adolescence was undertaken by Schilling et al. (2002). Concepts that evolved through the process of analysis are congruent with the broader concept of SM described in the IFSMT. Three significant attributes of SM were identified by Schilling et al.: process, activities, and goals. According to these investigators, successful diabetes management involves flexible, active and proactive processes; this process requires that children/adolescents and parents shift and share responsibilities (Schilling et al.). The process attribute of diabetes management in adolescence includes adaptation to changing physiology, shifting responsibilities, and decision making between the adolescent and parent(s) (Schilling et al.). This is similar to the "dynamic and fluid" nature of the sharing of responsibilities that occurs between individuals and family members with regards to disease SM as proposed in the IFSMT and the process dimension as outlined by Ryan and Sawin (2009).

According to Schilling et al. (2002), in order to successfully manage T1DM during adolescence, it is important for families to establish and maintain a plan to manage a multi-component diabetes treatment regimen encompassing the following activities: administration of insulin, multiple daily blood glucose determinations, dietary modification including limiting consumption of simple carbohydrates and monitoring overall carbohydrate intake, and carefully monitoring the effects of physical activities. With IFSMT, regimen specific activities are classified as proximal outcomes and regarded as SMB. Another attribute of SM posited by Schilling et al. are goals which 
include adolescents and their parents engaging in a goal setting process to accomplish certain purposes. Goal setting is considered a key process variable in IFSMT. Schilling et al. suggested there are three essential goals for individuals and families managing diabetes: carry out the prescribed diabetes regimen (SMB), maintain blood glucose levels within a normal range (achieve metabolic control), and "maintain life, health, and wellbeing" (p. 92). These correspond to the outcomes in IFSMT - achieving metabolic control, implanting SMB, and achieving DQOL. Schilling et al. added that parent and adolescent goals can differ in both emphasis and intent. Goals of diabetes management may at any given time be changing and differ from one parent/child dyad to another (Schilling et al.). IFSMT examines goal congruence and considers the issues and implications surrounding "the ability to resolve the confusion and anxiety occurring from apparently contradictory and competing demands associated with health goals" (Schilling et al., 2002, p. 225.e5).

The Self-Management of Diabetes in Adolescents (SMOD-A), a 52-item selfreport instrument created specifically for adolescents with T1DM was based on the concept analysis conducted by Schilling et al. (2002). The operationalization of the process attribute of diabetes SM in adolescence by Schilling et al. is similar to the "dynamic and fluid" nature of the sharing of responsibilities that occurs between individuals and family members with regards to disease SM as proposed in the IFSMT (Ryan and Sawin (2009). 


\section{Gaps in our Understanding of Self-management Behaviors and Health}

\section{Outcomes}

We have yet to develop a good understanding of how SMB are learned, maintained or adapted, or even discontinued over time. We don't know how context and process variables function in the daily life in adolescents with T1DM. Perhaps more importantly, we do not fully understand how SMB are related to health outcomes. Yet, the ability to adjust insulin, diet, or exercise to ever-changing individual daily situations is central to effective diabetes management and optimal diabetes outcomes. If the adolescent with T1DM and his/her family simply focus on adhering to a prescribed treatment plan without the ability to collaborate with care providers or reflect on various SBM options for successful problem resolution and do not have confidence to modify the treatment plan because of a changing social and/or personal situation, it is not clear that the desired outcomes can be achieved.

The evolution from adherence, compliance, and self-care to the concept of SM offers the opportunity to integrate multiple variables of interest in the study of T1DM outcomes. Currently, there remains a lack of understanding of the relationships between key variables in IFSMT. A growing body of science has emerged to support the direct and indirect relationships between context, process, and outcome variables specific to adolescents with T1DM yet we know very little about how SMB impact distal outcomes. There is an evolving body of evidence regarding process variables and their relationship to SM outcomes in adolescents with T1DM that will be further explored in Chapter 2. 


\section{Study Purpose and Aims}

The purpose of the proposed research study is to examine several aspects of the phenomenon of SM as described by Ryan and Sawin (2009) in adolescents with T1DM and identify factors that contribute to effective SM in adolescents with T1DM. There are two broad aims for this study: Test components of the IFSMT to better understand relationships between select context, process and outcome variables in adolescents with T1DM and their families, and determine whether SMB mediates the relationship of IFSMT process variables on metabolic control and HRQOL.

\section{Research Questions}

The following research questions consider the relationships among key variables in the IFSMT applied to adolescents with T1DM: 1) What is the relationship of key IFSMT context (e.g. regimen complexity, age, gender, depressive symptoms, and parent perceived life difficulty) and process variables (knowledge, self-efficacy, goal setting, problem solving, communication, collaboration, and autonomy support) on the IFSMT proximal outcome, SMB?

2) What is the relationship of key IFSMT context variables (e.g. regimen complexity, age, gender, depressive symptoms, and parent perceived life difficulty), process variables (knowledge, self-efficacy, goal setting, problem solving, communication, collaboration, and autonomy support), and the proximal outcome, SMB, on distal outcomes (HgbA1c and DQOL)?

3) Does SM (as a proximal outcome) mediate the relationship of select process variables on two distal outcomes: metabolic control and DQOL? 


\section{Orientation to the Dissertation}

The following chapters in this manuscript-option dissertation, Testing Components of a Self-Management Theory in Adolescents with Type 1 Diabetes, encompass: (a) three manuscripts, (b) a literature review pertinent to adolescents with T1DM and concepts from the IFSMT, (c) a methods review for handling missing data, (d) findings related to a correlational, cross-sectional study of factors associated with SMB and distal outcomes in 103 parent-adolescent dyads, and (e) discussion of implications for theory development, practice, research, and policy.

Chapter 2 is a manuscript describing current evidence related to SM in adolescents with T1DM using IFSMT as the framework. This chapter establishes the groundwork for the analysis of factors contributing to SMB and outcomes. At the request of the journal editor, the manuscript was formulated to assist practicing clinicians in applying current evidence relevant to context, process, and outcome variables in adolescents with T1DM. IFSMT is used to guide practicing clinician's understanding of SM from the context of the adolescent with T1DM and provides structure for enhancing diabetes care provided to adolescents with T1DM and their families. Interventions addressing the process dimension are highlighted for the clinician.

In Chapter 3, empirical and theoretical approaches for selecting methods to manage missing data in survey research designs are described in manuscript format. Various approaches for managing missing data are examined and newer techniques are endorsed. Missing data from this research study serve as an exemplar, demonstrating a 
realistic example of how to manage the problem of missing data when encountered in survey research studies.

Chapter 4 includes the third manuscript, the main study findings. The demographics of the study population along with survey measures are detailed. The quantitative methodology is described and an analysis addressing the primary study questions is included. Direct and mediating relationships between context, process, and outcome variables are examined. A discussion related to the findings, implications for nursing practice, study limitations and future research is included in this chapter.

Chapter 5 synthesizes implications for nursing practice, study limitations, exploration of a policy issue prompted by study findings, and ideas for future research. The findings explored in this chapter highlight a variety of issues and areas that need to be further addressed in the practice setting and through future research endeavors. 


\section{Reference List}

Amiel, A. A., Sherwin, R. S., Simonson, D. C., Lauritano, A. A., \& Tamborlane, W. V. (1986). Impaired insulin action in puberty: A contributing factor to poor glycemic control in adolescents with diabetes. The New England Journal of Medicine, 315, 215-219.

Anderson, B. (2009). Psychosocial care for young people with diabetes. Pediatric Diabetes, 10 (Suppl. 13): 3-8.

Anderson, B. J., Ho, J., Brackett, J., Finkelstein, D., \& Laffel, L. (1997). Parental involvement in diabetes management tasks: Relationships to blood glucose monitoring adherence and metabolic control in young adolescents with insulindependent diabetes mellitus. Journal of Pediatrics, 130, 257-265.

Anderson, B. J., Vangsness, L., Connell, A., Butler, D., Goebel-Fabbri, A., \& Laffel, L. M. B. (2002). Family conflict, adherence, and glycaemic controlin youth with short duration Type 1 diabetes. Diabetes Medicine, 19, 635-642

Auslander, W. F., Thompson, S., Dreitzer, D., White, N. H., \& Santiago, J. V. (1997). Disparity in diabetes control and adherence between African-American and Caucasian youths with diabetes. Diabetes Care, 20, 1569-1575.

Burroughs, T. E., Pontius, S. L., \& Santiago, J. V. (1993). The relationship among six psychosocial domains, age, health care adherence, and metabolic control in adolescents with IDDM. The Diabetes Educator, 19, 396-402. 
Chien, S. C., Larson, E., Nakamura, N., \& Lin, S. J. (2007). Self-care problems of adolescents with type 1 diabetes in southern taiwan. Journal of Pediatric Nursing, 22: 404-409.

DCCT. (1993). The effect of intensive treatment of diabetes on development and progression of long-term complications in insulin-dependent diabetes mellitus. The New England Journal of Medicine, 329, 977-986.

DCCT. (1994). Effect of intensive diabetes treatment on the development and progression of long-term complications in adolescents with insulin-dependent diabetes mellitus: Diabetes control and complications trial The Journal of Pediatrics, 125, 177-188.

DiMatteo, M. R. (2004). Social support and patient adherence to medical treatment: A meta-analysis. Health Psychology, 23, 207--218.

Ellis, D. A., Podolski, C-L., Frey, M., Naar-King, S., Wang, B., Moltz, K. (2007). The role of parental monitoring in adolescent health outcomes: Impact on regimen adherence in youth with Type 1 Diabetes, 32: 907-917.

JDRF (Juvenile Diabetes Research Foundation). (2009). What is type 1 diabetes? from http://www.jdrf.org/index.cfm?page_id=101982.

Goodall, T. A., \& Halford, W. K. (1991). Self-management of diabetes: A critical review. Health Psychology, 10, 1-8.

Goran, M. I., \& Gower, B. A. (2001). Longitudinal study on pubertal insulin resistance. Diabetes 50, 2444-2450. 
Grey, M., Davidson, M., Boland, E. A., \& Tamborlane, W. V. (2001). Clinical and psychological factors associated with achievement of treatment goals in adolescents with diabetes mellitus. Journal of Adolescent Health, 28, 377-385.

Hanna, K. M., \& Guthrie, D. (2003). Parental involvement in adolescents' diabetes management. Diabetes Spectrum, 16, 184-187.

Hanson, C. L., Henggeler, S. W., \& Burghen, G. A. (1987). Model of associations between psychosocial variables and health-outcome measures of adolescents with IDDM. Diabetes Care, 10, 752-758.

Harris, M. A., Wysocki, T., Sadler, M., Wilkinson, K., Harvey, L. M., Buckloh, L. M., ...White, N. H. (2000). Validation of a structured interview for the assessment of diabetes self-management. Diabetes Care, 23, 1301-1304.

Hoffman, R. P. (2002). Adolescent adherence in Type 1 diabetes. Comp Therapy, 28, 128-133.

Iannotti, R. J., Nansel, T. R., Schneider, S., Haynie, D. L., Simons-Morton, B., Sobel, D. O., . . Clark, L. (2006). Assessing regimen adherence of adolescents with type 1 diabetes. Diabetes Care, 29, 2263-2267.

Karvonen, M., Viik-Kajander, M., Moltchanova, E., Libman, I., LaPorte, R., Tuomilehto, J., \& al., e. (2000). Incidence of childhood Type 1 diabetes worldwide. Diabetes Care, 23, 1516-1526.

King, P. S., Berg, C., Butner, J., Butler, J. M., \& Weibe, D. J. (2013). Longitudinal trajectories of parental involvement in Type 1 diabetes and adolescents' 
adherence. Health Psychology. Advance online publication. doi: 10.1037/a0032804.

La Greca, A. M., Follansbee, D., \& Skyler, J. S. (1990). Developmental and behavioral aspects of diabetes management in youngsters. Children's Health Care, 24, 132139.

Leonard, B. J., Garwick, A., \& Adwan, J. Z. (2005). Adolescents' perceptions of parental roles and involvement in diabetes management. Journal of Pediatric Nursing, 20, 405-414.

Lewin, A. B., Storch, E. A., Williams, L. B., Duke, D. C., Silverstein, J. H., \& Geffken, G. R. (2010). Brief report: Normative data on a structured interview for diabetes adherence in childhood Journal of Pediatric Psychology, 35, 177-182.

Miller, V. A., \& Drotar, D. (2003). Discrepancies between mother and adolescent perceptions of diabetes-related decision-making autonomy and their relationship to diabetes-conflict and adherence to treatment Journal of Pediatric Psychology, $28,265-274$.

Moran, A., Jacobs, D. R., Steinberger, J. H., C. P. Prineas, R., Luepker, R., \& Sinaiko, A. R. (1999). Insulin resistance during puberty: Results from clamp studies in 357 children Diabetes 48, 2039-2044.

Pereira, M. G., Berg-Cross, L., Almeida, P., \& Machado, J. C. (2008). Impact of family environment and support on adherence, metabolic control, and quality of life in adolescents with diabetes. International Journal of Behavioral Medicine, 15, 187193. 
Ryan, P., \& Sawin, K. J. (2009). The individual and family self-management theory: Background and perspectives on context, process, and outcomes. Nursing Outlook, 57, 217-225.

Rydall, A. C., Rodin, G. M., Olmsted, M. P., Devenyi, R. G., \& Daneman, D. (1997). Disordered eating behavior and microvascular complications in young women with insulin-dependent diabetes mellitus. The New England Journal of Medicine, $336,1849-1854$.

Schilling, L. S., Grey, M., \& Knafl, K. A. (2002). The concept of self-management of type 1 diabetes in children and adolescents: An evolutionary concept analysis. Journal of Advanced Nursing, 37, 87-99.

Skinner, T. C., \& Hampson, S. E. (2001). Personal models of diabetes in relation to selfcare, well-being, and glycemic control. A prospective study in adolescence. Diabetes Care, 24: 828-833.

Streisand, R., \& Mednick, L. (2006). Development of the diabetes education, counseling, information delivery and evaluation (DECIDE) program: A health promotion intervention for preadolescents with Type 1 diabetes. Journal of Clinical Psychology in Medical Settings, 13, 189-199. doi: 10.1007/s10880-006-9019-6.

Urban, A.D. \& Grey, M. (2006). Type 1 diabetes. Nursing Clinics of North America, 41, 513-530.

Weibe, D. J., Berg, C. A., Korbel, C., Palmer, D. L., Beveridge, R. M., \& Upchurch, R. (2005). Children's appraisals of maternal involvement in coping with diabetes: 
Enhancing our understanding of adherence, metabolic control, and quality of life across adolescence. Journal of Pediatric Psychology, 30, 167-178.

Weinger, K., Butler, H., Welch, G., \& La Greca, A. (2005). Measuring diabetes self-care: A psychometric analysis of the self-care inventory-revised with adults Diabetes Care, 28, 1346-1352.

White, N. H., Cleary, P. A., Dahms, W., Goldstein, D., Malone, J., \& Tamborlane, W. V. (2001). Beneficial effects of intensive therapy of diabetes during adolescence: Outcomes after the conclusion of the Diabetes Control and Complications Trial (DCCT) Journal of Pediatrics, 139, 804-812.

Wysocki, T., Buckloh, L. M., Anal, H., Lochrie, A., \& Taylor, A. (2012). Validation of a self-report version of the diabetes self-management profile. Pediatric Diabetes, $13,432-437$.

Wysocki, T., Taylor, A., Hough, B. S., Linscheid, T. R., Yeates, K. O., \& Naglieri, J. A. (1996). Deviations from developmentally appropriate self-care autonomy: Association with diabetes outcomes. Diabetes Care, 19, 119-125. 


\title{
Chapter 2
}

\section{Optimizing Self-management in Adolescents with T1DM}

\begin{abstract}
Purpose: To use concepts from the Individual and Family Self-Management Theory (IFSMT) as a guide for clinicians' assessment and interventions when caring for adolescents with Type I Diabetes Mellitus (TIDM) and their families. Design and Methods: A review of the literature of self-management (SM) in adolescents with TIDM was conducted. Results: Key IFSMT context and process variables helpful in understanding optimal outcomes (SMB, metabolic control, and quality of life) are presented. Practice Implications: IFSMT can provide structure and guidance for understanding SM in adolescents with T1DM.
\end{abstract}

Keywords: Type 1 Diabetes Mellitus, adolescence, self-management, interventions, theory 
Advancing treatment technologies and understanding the significant role that normoglycemia plays in preventing chronic complications associated with T1DM has shifted the emphasis of diabetes management from a medically managed approach to one where a more collaborative relationship exists between health care provider and the adolescent with T1DM and his/her family (Schilling, Grey \& Knafl, 2002). The primary responsibility for managing T1DM rests with the parent during childhood. However, during adolescence, increasing levels of responsibility for SM are transferred to the adolescent. Understanding factors related to SMB and the impact of SMB on health outcomes will enable nurses across care delivery settings to support this important outcome for adolescents with diabetes and their families.

A middle range theory, Individual and Family Self-management Theory (IFSMT) developed by Ryan and Sawin (2009) can provide clinicians a framework for assessing, planning, and implementing a theory based approach to care for adolescents with T1DM and their families in order to facilitate optimal diabetes SM outcomes. This manuscript examines essential elements of IFSMT and provides a synthesis of SM literature related to adolescents with T1DM. A review of intervention studies is included to assist the clinician to further evaluate the impact of implementing various process related strategies.

\section{IFSMT as a Frame work for Practice}

IFSMT conceptualizes SM as a process by which individuals and families use knowledge and beliefs, self-regulation skills and abilities, and social facilitation to achieve proximal (e.g., SM behavior $[\mathrm{SMB}]$ ) and distal outcomes (health status, quality 
of life (QOL) and cost of health) (Ryan \& Sawin, 2009). According to IFSMT, SM takes place in the context of risk and protective factors specific to a particular health condition, the physical and social environment and various individual and family factors (see Figure 1). $\mathrm{SM}$ is conceptualized as:

A process in which individuals and families use knowledge and beliefs, selfregulation skills and abilities, and social facilitation to achieve health-related outcomes. Self-management takes place in the context of risk and protective factors specific to a health condition, physical and social environment and individual and family. Self-management is applicable to chronic conditions as well as health promotion" (UWM SMSC, 2011; Ryan and Sawin; 2009, Ryan 2009).

Ryan and Sawin contend that researchers have traditionally studied SM from either an "individual lens" or "family lens" but have not viewed individuals and families collectively. They maintain that using both lenses concurrently allows for a more "comprehensive perspective" and an ability to detect the changing dynamics within an individual and family system.

Applying concepts from IFSMT to adolescents with T1DM can help the clinician provide a more focused assessment and use evidenced-based interventions to optimize outcomes, SMB, hemoglobinA1c (HgbA1c) and diabetes-specific health related quality of life (DQOL). Further, IFSMT proposes that the more "proximal" or preliminary SMB outcome, which is the focus of many nursing interventions, leads to later or "distal" outcomes: improved health status (HgbA1c) and improved DQOL for adolescents with TIDM and ultimately lower costs associated with managing T1DM over time. Even though the effectiveness of SM interventions in the clinical setting have yet to be fully tested, attention to these clinical variables by the clinician is warranted. There is a 
growing body of evidence to support the application of these interventions in every day clinical practice.

\section{Context Factors and their Relationship to Self-Management Behaviors}

According to IFSMT, contextual factors can be viewed as either risk or protective factors that contribute to SMB. Key context factors impacting adolescents with T1DM and their families are represented within three categories: condition-specific context factors, physical and social environment, and individual and family factors.

Condition-specific contextual factors. Condition-specific factors address the individual and family's perception of the complexity associated with T1DM, the complexity of the treatment regimen, the condition stability and disease trajectory. A factor influencing both complexity and condition stability involves the changing needs for insulin during the 'honeymoon' phase. With new onset T1DM, adolescents can present with signs and symptoms ranging from hyperglycemia and mild ketosis to full blown diabetic ketoacidosis (DKA) and metabolic decompensation. After correcting the metabolic abnormalities, a significant proportion of newly diagnosed individuals regain the ability to secrete some insulin from the remaining functional $\beta$-cells. This transient phase is referred to as the "honeymoon" or partial remission, characterized by continued

endogenous insulin secretion. As a result, insulin needs decrease and most youth require only a very small amount of exogenous insulin to normalize and maintain blood glucose (BG) in a normal/near-normal range. As the honeymoon period draws to a close, endogenous insulin production starts to phase out and individuals with T1DM experience fluctuating BG levels signifying unstable insulin production. This can be a stressful time 
for both the adolescent with T1DM and family members as they attempt to correct the often unanticipated erratic BG levels.

Keeping up with increasing insulin requirements related to hormonal changes associated with puberty is a major factor preventing adolescents from being able to obtain good metabolic control (Goran \& Gower, 2001). In both healthy and diabetic adolescents, insulin sensitivity decreases during puberty, thus further contributing to the difficulties adolescents experience in achieving optimal metabolic control (Amiel et al., 1986, Moran et al., 1999; Szadkowska et al., 2007).

The complexity associated with the diabetes treatment regimen can be daunting for adolescents and their parents. Participating in an intensive management protocol means being constantly aware of how the multi-component treatment regimen of dietary intake, exercise, illness and stress is affecting BG levels and making decisions regarding insulin doses based on a host of factors (Silverstein et al., 2005). Intensive management mandates anticipating and monitoring BG levels numerous times throughout the day in order to assess daily metabolic control, administering multiple daily injections of insulin or using a continuous subcutaneous insulin infusion (CSII, often called the "pump") to provide sufficient insulin coverage in response to glucose excursions and meet the 24hour continuous maintenance needs for basal insulin.

Insulin therapy raises the insulin concentration in the blood stream and increases the risk for hypoglycemia, thus affecting the perception of condition stability. Hypoglycemic episodes are an extant and significant risk associated with intensive management of T1DM. Severe hypoglycemia was determined to be two to four times 
greater in adolescents in the intensive treatment arm of the DCCT (1994) yet there were no differences found in neuropsychological functioning or HRQOL scores between intensively and conventionally managed youth (DCCT). Researchers have concluded that the benefits of tight BG control outweighed the risks associated with an acute hypoglycemic crisis in intensively managed individuals (White et al., 2001).

Physical and social environment. Adolescents spend much of their time attending school. School personnel are required by federal law (Individuals with Disabilities Education Act Amendments, 1997; Vocational Rehabilitation Act of 1973, Section 504) to develop an individual assessment plan addressing the unique needs of youth with diabetes. School personnel need to have a basic understanding of T1DM and the needs of students with T1DM to ensure adolescents and parents that school is a safe and healthy environment. Nichols and Norris (2002) suggest teachers often lack adequate knowledge or training to facilitate optimal care for adolescents, school nurses aren't always readily available to assist in providing for the acute needs of adolescents with T1DM, and school rules can hamper optimal self-care.

Individual and family factors. Individual and family factors found to impact outcomes in adolescents with T1DM include age, developmental stage, gender, socioeconomic status, literacy, mental health, family cohesion and family structure. Age appears to be related to SMB, but the relationship in adolescence is a complex one and could be confounded by other variables including bio-physical development and complexity of the condition. Urbach et al. (2005) found age and marital status of 
biological parents predicted metabolic control in adolescents with T1DM; adolescents 1418 years of age had the highest mean HgbA1c $(9.7 \pm 1.5)$.

Adolescent developmental theory suggests that one of the major tasks of adolescence involves the adolescent moving away from dependence on family, yet not toward independence from family, but toward an interdependent relationship between the adolescent and family (Baumrind, 1991). This interdependence requires a reorganization process in which family members renegotiate responsibilities (Anderson Ho, Brackett, Finkelstein, \& Laffel, 1997; Weibe et al, 2005). The parent role transforms from 'doing for' the adolescent to more of an advisor/mentor role. Anderson et al. demonstrated that parent engagement with the adolescent, rather than separation from the adolescent increased adolescent SMB and in turn improved metabolic control.

Within the clinical context of diabetes, gender differences have been identified. In general, female adolescents experience worse metabolic control, have more depression and anxiety and a reduced DQOL. In the Hvidore Study Group on Childhood Diabetes, a multicultural cohort study involving 18 countries covering three continents, females demonstrated increased worries, less life satisfaction, and poorer health perception (Hoey et al., 2001). Consistent with these findings, Faulkner (2003) found females with T1DM had lower life satisfaction scores and those in middle adolescence experienced lower life satisfaction than those in late adolescence. Hanberger et al. (2009) found girls reported lower general and DQOL.

Socioeconomic status is one of the most frequently identified predictors of metabolic control. In general terms, youth from lower income families are more likely to 
experience poorer metabolic control than their peers with higher SES (Campbell et al., 2014). With regards to ethnicity and racial differences, studies have shown that AfricanAmerican youths experience poorer metabolic control than Caucasians (Faulkner \& Chang, 2007; Greening, Stoppelbein, Konishi, Jordan \& Moll, 2006).

In order for individuals with T1DM to execute complex, intensive treatment recommendations, literacy and numeracy skills are essential. Limited health literacy has been linked to reduced diabetes knowledge and worse metabolic control (Gazmararian, Williams, Peel, \& Baker, 2003; Schillinger et al., 2002). Cavanaugh et al. (2008) found that despite satisfactory literacy skills, adults with diabetes demonstrated low-level numeracy skills (math skills at less than a ninth-grade level). For individuals on insulin in Cavanaugh's study, low diabetes numeracy was associated with reduced participation in SMB.

Multiple studies in the literature indicate that depressive symptoms are a frequent problem in those with T1DM. Hood et al. (2006) found 14\% of adolescents with TIDM had depressive symptoms while Grey and colleagues identified a 20\% prevalence of depressive symptoms compared to $7 \%$ in non-diabetic peers (Grey, Whittemore \& Tamborlane, 2002). The development of these depressive symptoms begins early with children 8-14 years of age and two years post diagnosis reporting twice the depressive symptoms than their peers (Grey, Cameron, Lipman \& Thurber, 1995).

Burroughs, Harris, Pontius, and Santiago (1997) examined family characteristics from over 30 studies and found that supportive, cohesive families were more likely to have adolescents with stronger SMB and metabolic control than families that did not 
demonstrate such cohesion. Several researchers emphasized the critical role support plays during the period of time between diagnosis and the first few years after diagnosis because positive early adjustment is predictive of better outcomes in later adolescence (Burroughs et al, Anderson et al., 2002).

The effects of family structure on T1DM outcomes indicate that adolescents from families where parents lived together equated to improved metabolic control and DQOL when compared with adolescents who were living with parents in a separated or singleparent arrangement (Hanberger et al., 2009). Cameron et al. (2008) observed lower hemoglobin A1c (HgbAlc) levels in families where the father was employed, yet for mothers in the study, no relationship between employment status and metabolic control was found.

\section{Process Factors and their Relationship to Self-Management Behaviors}

In the following section, concepts relevant to the process of SM are outlined. Significant process interventions and associated outcomes obtained from a review of the literature are illustrated in Table 1.

Knowledge and beliefs. Knowledge alone does not impact behavior but a critical level of knowledge is necessary to develop self-efficacy (SE) as well as the more advanced self-regulation skills and abilities. Knowledge is a building block in diabetes education. In order to cope with the complex, multifaceted demands of the diabetes treatment regimen, a reasonable sense of SE is necessary. SE has been correlated with SMB; greater SE has been shown to predict higher levels of responsibility for diabetes related tasks and treatment regimens (Littlefield et al., 2002; Ott, Greening, Palardy, 
Holderby, \& DeBell, 2000). Johnston-Brooks, Lewis, and Garg (2002) examined SE and self-esteem as aspects of self that influence self-care and metabolic control among young adults with T1DM and found SE to be a better predictor of SMB and HgbA1c than selfesteem.

Se If-regulation skills and abilities. In order for adolescents and family members to initiate effective SMB and cope with the challenges associated with T1DM, they need to gain skills and abilities through participation in quality diabetes self-management education. Current best practice of diabetes education is a skills based approach, helping individuals and families make informed SM choices (Marraro et al., 2013). This skillsbased education model has improved SMB and metabolic control (Grey, Boland, Davidson, Li, \& Tamborlane, 2000; Lorig, Sobel, Ritter, Laurent, \& Hobbs, 2003). Adolescents with T1DM are constantly bombarded with challenging demands associated with diabetes management. Adjusting to these demands can be taxing for both the adolescent and family. It is important to understand the strategies adolescents use to cope with these day-to-day challenges. With a program of research focused on Coping Skills Training (CST) in adolescents and/or parents, Grey and associates have contributed to furthering our understanding of the concept of coping and how enhancing coping skills can improve clinical outcomes through interventions focused on process skills and abilities (Grey et al., 1998; Grey, Boland, Davidson, Li, \& Tamborlane, 2000; Grey et al., 2009; Grey, 2011). These interventions include reflective thinking, self-monitoring, problem solving and managing emotions (see Table 1). 
Social facilitation. Adolescents are more likely to engage in appropriate SMB if they experience social facilitation that positively influences and supports SMB. Drew, Berg \& Weibe (2010) studied whether increased adolescent peer orientation impacted SMB and metabolic control. Drew et al. found adolescents with higher quality parent relationships demonstrated better SMB and metabolic control- the converse occurred with increased adolescent peer orientation. Hains et al. (2007) found that when adolescents with T1DM perceive negative attributions regarding friends and peer reactions to SM in social situations it impacted SM difficulties, which in turn increased diabetes stress, which had a direct effect on metabolic control. Interestingly, as friend support increased in Hains et al. study, the relationship between stress and metabolic control also increased.

Hanna and Guthrie (2000a, 2000b) examined perceived parent and adolescent benefits and barriers related to the transfer of SM responsibility that occurs during adolescence. Adolescents perceived that when they were more involved in diabetes SM, parents were relieved of responsibility, worry, and stress. Perceived SM benefits by adolescents in the study included having knowledge of and confidence in SM abilities and approval from family members to experience more freedoms associated with being a teenager. Additionally, feeling the burden of personal responsibility was a significant barrier to SM for the adolescent (Hanna \& Guthrie, 2000a). Parents perceived a sense of relief with the transfer yet acknowledged the loss of control and angst associated with having to deal with the consequences of possible mismanagement (Hanna \& Guthrie, 2000b). 
Subsequent to their work on perceived benefits and barriers associated with SM responsibilities, Hanna and Guthrie (2001) examined parents' and adolescents' perceptions of helpful and non-helpful dimensions of support related to adolescents' assuming diabetes management responsibilities. Overall study findings indicated that adolescents still want parents to assist with aspects of SM. Whether parental guidance was helpful to the adolescent depended on the degree of directness and the perceived need for help. Adolescents recommended that parents maintain warm, caring relationships with them and use "subtle techniques" of guidance including reasoning, suggesting, and listening.

Numerous studies have examined negotiated collaboration; several researchers have been able to demonstrate that greater parental involvement is associated with improved self-management behavior on the part of the adolescent (Anderson et al., 1997; Leonard, Garwick, \& Adwan, 2005) and ultimately improved diabetes control (Leonard et al., 2005). Conversely, other researchers have failed to demonstrate a relationship between parental responsibility and improved adolescent self-management behaviors (Miller \& Drotar, 2003; Weibe et al., 2005) or improved metabolic control (Anderson et al., 1997; Miller \& Drotar, 2003; Weibe et al., 2005).

Diabetes related communication between the adolescent with T1DM and his/her parents are an integral part of negotiated collaboration. Increasing parent involvement can create an increase in parent and adolescent conflict regarding diabetes SM. Daschiff, Hardeman and McLain (2008) studied communication regarding diabetes management between adolescents with T1DM and their parents and suggest parent/adolescent 
communication can be perceived in one of two ways by the adolescent: supportive or conflict generating. Conflict generating communications are perceived by the adolescent as the parent placing blame on the adolescent for mismanagement, failing to understand difficulties associated with SM, conveying expressions of worry through "intrusiveness", or focusing on the future in a way that is perceived as worrisome to the adolescent.

\section{Process Related Interventions}

Although intervention studies aim to optimize SMB can address both context and process variables, typically the major focus for adolescents with T1DM is on process variables. Nurses and other health care providers need to be aware of the context (is the adolescent on a pump or injection, what is their school setting, access to resources) and individualize interventions aimed at knowledge, SE, self-regulation and social facilitation based on these context variables. In some cases the clinician may work to change the adolescent's context to reduce factors such as depressive symptoms or a physical environment that interferes with developing the skills and abilities foundational to SMB. Table 1 includes a summary of process-related interventions designed to enhance SM outcomes reported in the literature between 2000 and 2012. The table is organized by delivery method: Interventions delivered in a traditional face to face method or by telephone are identified in the first cluster followed by interventions delivered in the Internet environment. Interventions are organized by categories within the process dimension; often the study's focus encompasses several categories within a process variable. 


\section{Outcomes}

Consistent with IFSMT, engaging in SMB is the proximal outcome of interest for adolescents with T1DM and their families. A significant gap in our knowledge of SM surrounds SMB and how these behaviors affect outcomes. In part, this is due to the conceptual confusion surrounding the terms used to describe SM. Historically, SM has been not been clearly defined; often referred to as adherence or self-care. Therefore we have lagged in our development of instruments that measure SM and subsequently this has directly impacted our ability to 'test' and understand relationships between concepts of interest.

Distal outcomes include health status, QOL, and costs associated with care (Ryan \& Sawin, 2009). Metabolic control has been the most studied distal outcome measure of SM in adolescents. The study of the relationship(s) between context/process variables and metabolic control remains controversial; confirmed by some researchers and refuted by others.

When comparing DQOL in adolescents with T1DM to their healthy (nondiabetic) peers, there is evidence of inconsistent findings. Several researchers have found that adolescents with T1DM report no difference or better DQOL than their peers (Hesketh, Wake, \& Cameron, 2004; Laffel et al., 2003; Wagner, Mueller-Goddeffroy, von Sengbusch, Hager, \& Thyen, 2005). Varni et al. (2003) found adolescents reported worse psychosocial health, emotional functioning and school functioning on generic QOL indicators than their (non-diabetic) peers. 
Several researchers compared DQOL ratings from parents of adolescents with T1DM and parents of healthy adolescents and found parents of adolescents with T1DM rated their adolescent's health worse than parents ratings of their healthy children (Hesketh et al., 2004; Laffel et al., 2003; Varni et al., 2003). In a study comparing adolescent and parent ratings regarding adolescent DQOL, De Wit et al. (2007) found moderate to high agreement between parent and adolescent scores, especially with regard to physical well-being. Additionally, adolescents (13-16.5 years of age) rated less behavioral problems when compared to parent reports.

In the majority of intervention studies found in Table 1 where QOL was a criterion variable, the measure used was diabetes-specific. One study included only a generic QOL measure (Grey et al., 2013) and one study incorporated both generic and diabetes specific QOL indicators as predictor variables (de Wit et al., 2007, 2008).

\section{Critique of the Lite rature}

A majority of studies referenced within this review were descriptive. The samples included adolescents with T1DM and their parents, families and peers. Most of these studies were cross-sectional with a primary aim of investigating associations between study variables. Fewer studies were designed as longitudinal studies. Although we have yet to fully understand how SM is achieved in the daily life of the adolescent with T1DM, there is an emerging body of evidence that suggests direct and indirect relationships between context, process, and outcome variables proposed by the IFSMT.

Much of what is known about the relationship between process variables and distal outcomes such as HgbA1c and QOL has evolved from randomized controlled trials 
with interventions delivered by clinicians specializing in diabetes, conducted in person, within groups, in a structured environment. The authors of this body of literature (11 randomized/non-randomized controlled studies and 3 single group designs, see Table 1) systematically evaluated behavioral, educational, psychosocial or family interventions aimed primarily at the process of SM. Most of the studies were large enough and sufficiently powered to evaluate the efficacy of the proposed interventions in a research setting.

The interventions tested addressed reflective thinking, self-monitoring/se lfregulation, problem solving, self-efficacy, managing emotions and parent-adolescent communication. The majority of the studies, especially those in the program of research by Grey and colleagues, found interventions aimed at strengthening these process variables yielded improvements in HgbA1c (see Table 1) and some reported positive impact on quality of life. Only one study (Ellis et al., 2007) reported costs associated with implementing the intervention. Some of these interventions are currently being integrated into best practices in diabetes SM programs. It remains unclear how these structured, group interventions translate into 'typical' daily practices of individual providers.

There have not been sufficient trials which evaluate the effectiveness of these interventions in clinical practice. Particularly important would be evaluation of these interventions when delivered by health care providers in specialty or primary care. Whittemore and Grey (2002) assert that effectiveness clinical trials should follow efficacy research as a "critical next step" to better understanding the robustness of an 
intervention study under more typical practice conditions. Programs of research that focus on clinical effectiveness are necessary to continue to advance the science of SM in adolescents with T1DM.

\section{Implications for Practice}

Based on findings from this review, the following select nursing actions and interventions are recommended to enhance care and clinical outcomes for adolescents with T1DM and families across the continuum of health care settings. When planning care for adolescents with T1DM, it is important for the nurse to arrange time to meet with the adolescent-parent dyad together and plan time to meet alone with the adolescent in order to obtain a comprehensive assessment and increase opportunities to observe and participate in adolescent and parent/family interactions. Care begins with a detailed assessment and an important part of the assessment includes garnering an understanding of both adolescent and parent perspectives regarding the adolescent's SMB and DQOL. Asking focused assessment questions about various aspects of the context, process, and SMB can pinpoint where potential difficulties lie and target specific interventions. Helpful indicators for assessing DQOL in adolescents include physical aspects associated with the experience of T1DM, psychosocial and emotional health, social health, and school functioning. Not only does a DQOL assessment help the nurse gather essential information, it can also serve as a valuable intervention tool. Taking time to better understand some of the difficulties the adolescent and family encounter as they deal with the day-to-day issues surrounding the management of T1DM and exploring possible solutions can enhance negotiated collaboration and ultimately improve DQOL. 
Depression is not an easy disorder to recognize in youth. Depression has been found to be more common in adolescents with T1DM than in their non-diabetic peer group (Grey, Whittemore \& Tamborlane, 2002; Hood et al., 2006). It is important to assess for depression/ depressive symptoms in youth with T1DM because these symptoms can interfere with SM. Corathers et al. (2013) describe a systematic approach for routine depression screening in clinical practice using the Children's Depression Inventory. Nurses caring for adolescents with T1DM should screen for depression/depressive symptoms, have knowledge of mental health resources, and be ready to refer at-risk adolescents to mental health professionals skilled in treating depression/depressive symptoms.

In order to promote self-regulation, it is important for the nurse to help the adolescent and family set SM goals. Achieving goals that are realistic, timely, and attainable can promote SE. Recognizing that families who maintain parental involvement in supervising their adolescent's SMB are more likely to achieve positive diabetes-related outcomes, it is important for the nurse to work with both the adolescent and family members. Encouraging parental involvement in developing skills and abilities related to self-monitoring, problem solving, and disease management can facilitate improved SMB.

The majority of diabetes education occurs in the outpatient setting—even at diagnosis where intensive outpatient education may occur. A factor influencing both complexity and condition stability involves the changing insulin requirements during the 'honeymoon' phase. Exogenous insulin needs typically decrease during this phase and most youth require only a very small amount of insulin to normalize and maintain blood 
glucose $(\mathrm{BG})$ in a normal/near-normal range. Adolescents with newly diagnosed T1DM and family members need to understand this phenomenon, develop self-regulation skills and abilities associated with managing hypoglycemia, and maintain close contact with clinicians to facilitate effective decision making related to changing insulin needs.

For most adolescents with T1DM hospitalization occurs in a crisis situation. However, these crisis situations, once stabilized, can be the impetus to consider developing new skills. Individualized assessments and interventions to enhance skills in self-efficacy, problem solving and coping can be implemented during these hospitalizations. Coordination with nurses in the outpatient setting can optimize the continued development of these skills.

A nurse responsible for diabetes education might consider investigating whether there are opportunities to develop weekly, monthly, or quarterly small group sessions that families could participate in for the purpose of garnering support and learning vicarious from each other. A select T1DM topic of interest related to skills and knowledge development could be offered on a rotating basis. Developing a 'tool kit' containing scenarios that focus on typical problematic social situations adolescents and families encounter and using these scenarios to role play with the adolescent and family could prove to be a timely and effective intervention.

Figure 2 is a compilation of key context, process and outcome factors from IFSMT designed to guide nurses as they care for adolescents with T1DM and their families. According to IFSMT, SM takes place in the context of risk and protective factors specific to a health condition (like T1DM), the physical and social environment, 
and unique individual and family factors (Ryan \& Sawin, 2009). Including key context and process variables in the nursing assessment and plan of care for adolescents increases the likelihood of achieving proximal (SMB) and distal outcomes (HgbA1 and DQOL).

\section{Conclusions}

We are beginning to understand the promising role process variables play in optimizing SMB and health outcomes for adolescents with T1DM and their families. It is clear that without including key process variables in the SM equation, we cannot fully understand how two individuals and/or families with similar chronic health conditions have very different outcomes, one doing well and the other poorly. Equally important to achieving SM outcomes, interventions need to be carefully crafted, attending to designs that include not only the adolescent but incorporating family into the equation as well. We are just beginning to learn how technology can be used as a tool to enhance access, diabetes self-management education programming, which in turn can impact SMB and health outcomes. Combining cutting edge technologies that incorporate key processrelated interventions has the potential to revolutionize diabetes care.

IFSMT can serve as a guiding framework for clinicians caring for adolescents with T1DM because it offers a structure for understanding individual and family SM by including both context and process variables of adolescents and family members living with a diagnosis of T1DM. By enhancing our understanding of how key variables affect SM and how SM can affect client outcomes, IFMST can serve as a cogent model for clinical practice and the basis for expanding upon the current science of diabetes SM. 


\section{Reference List}

Amiel, S.A., Sherwin, R. S., Simonson, D. C., Lauritano, A. A., Tamborlane, W.V. (1986). Impaired insulin action in puberty. A contributing factor to poor glycemic control in adolescents with diabetes. New England Journal of Medicine, 315: 215219.

Anderson, B. (2009). Psychosocial care for young people with diabetes. Pediatric Diabetes, 10 (Suppl. 13): 3-8.

Anderson, B. J., Ho, J., Brackett, J., Finkelstein, D., \& Laffel, L. (1997). Parental involvement in diabetes management tasks: Relationships to blood glucose monitoring adherence and metabolic control in young adolescents with insulindependent diabetes mellitus. Journal of Pediatrics, 130, 257-265.

Anderson, B. J., Vangsness, L, Connell, A., Butler, D., Goebel-Fabbri, A., \& Laffel, L. M. B. (2002). Family conflict, adherence, and glycaemic control in youth with short duration Type 1 diabetes. Diabetes Medicine, 19: 635-642.

Baumrind, D. (1991). Effective parenting during the early adolescent transition. In P. E. Cowan \& E. M. Hetherington (Eds.) Advances in family research (Vol. 2, pp. 111-163). Hillsdale, NJ: Erlbaum.

Burroughs, T. E., Harris, M. A., Pontius, S. L., \& Santiago, J. V. (1997). Research on social support in adolescents with IDDM: A critical review. The Diabetes Educator, 23, 438-448. 
Cameron, F. J., Skinner, T. C., de Beaufortt, C. E., Hoey, H., Swifts, G. F., \& Aanstoot, H., et al. (2008). Are family factors universally related to metabolic outcomes in adolescents with Type 1 diabetes? Diabetic Medicine, 25,463-468.

Campbell, M. S., Schatz, D. A., Chen, V., Wong, J. C., Steck, A., Tamborlane, W. V.,...Haller, M. J. (2014). A contrast between children and adolescents with excellent and poor control: the TI1D exachage clinic registry expereince. Pediatric Diabetes, 15: 110-117.

Cavanaugh, K., Huizinga, M. D., Wallston, K. A., Gebretsadik, T., Shintani, A., Davis, D., . . Rothman, R. L. (2008). Association of numeracy and diabetes control. Annals of Internal Medicine, 148, 737-746.

Cespedes-Knadle, Y.M. \& Munoz, C. E. (2011). Development of a group intervention for teens with Type 1 diabetes. The Journalfor Specialists in Group Work, 36, 278-295.

Corathers, S. D., Kichler, J., Yayah Jones, N.-H., Houchen, M. J., Morwessel, N., Crawford...Hood, K.K. (2013). Improving depression screening for adolescents with Type 1 Diabetes. Pediatrics, 132, e1395-e1402.

Dashiff, C., Hardeman, T., \& McLain, R. (2007). Parent-adolescent communication and diabetes: an integrative review. Journal of Advanced Nursing, 62: 140-162 doi: 10.1111/j.1365-2648.2007.04549.x

DCCT. (1994). Effect of intensive diabetes treatment on the development and progression of long-term complications in adolescents with insulin-dependent 
diabetes mellitus: Diabetes control and complications trial The Journal of Pediatrics, $125,177-188$.

de Wit, M., Delemarre-van de Waal, H., Bokma, J. A., Haasnoot, K., Houdijk, M. C., Gemke, R. J., \& Snoek, F. J. (2007). Self-report and parent-report of physical and psychosocial well-being in Dutch adolescents with Type 1 diabetes in relation to glycemic control. Health and Quality of Life Outcomes, 5, 1-8.

De Wit, M., Delemarre-Van De Waal, H. A., Bokma, J. A., Haasnoot, K., Houdijk, M., Gemke, R. J., \& Snoek, F. J. (2008). Monitoring and discussing health-related quality of life in adolescents with Type 1 diabetes improve psychosocial wellbeing. Diabetes Care, 31, 1521-1526.

De Wit, M., Delemarre-Van De Waal, H. A., Bokma, J. A., Haasnoot, K., Houdijk, M., Gemke, R. J., \& Snoek, F. J. (2010). Follow-up results on monitoring and discussing health-related quality of life in adolescent diabetes care: benefits do not sustain in routine practice. Pediatric Diabetes, $11,175-181$.

Drew, L. M., Berg, C., \& Wiebe, D. J. (2010). The mediating role of extreme peer orientation in the relationships between adolescent-parent relationship and diabetes management. Journal of Family Psychology, 24, 299-306.

Ellis, D. A., Naar-King, Frey, M., Templin, T.,Rowland, M. \& Greger, N. (2004). Use of multisystemic therapy to improve regimen adherence among adolescents with Type 1 diabetes in poor metabolic control: A pilot investigation. Journal of Clinical Psychology in Medical Settings, 11, 315-324. 
Ellis, D. A., Templin, T., Naar-King, S., Frey, M., Cunningham, P. B., Podolski, C-L., \& Cakan, N.(2007). Multisystemic therapy for adolescents with poorly controlled Type 1 diabetes: Stability of treatment effects in a randomized controlled trial. Journal of Consulting and Clinical Psychology, 75: 168-174.

Faulkner, M. (2003). Quality of life for adolescents with Type 1 diabetes: Parental and youth perspective. Pediatric Nursing, 29, 362-368.

Faulkner, M. S. \& Chang, L.I. (2007). Family influence on self-care, quality of life, and metabolic control in school-age children and adolescents with Type 1 diabetes. Journal of Pediatric Nursing, 22, 59-68.

Faulkner, M. S., Michaliszyn, S. F., \& Hepworth, J. T. (2010). A personalized approach to exercise promotion in adolescents with type 1 diabetes. Pediatric Diabetes, 11 , 166-174.

Franklin, V. L., Waller, A., Pagliarit, C., \& Greene, S. A. (2006). A randomized controlled trial of Sweet Talk, a text-messaging system to support young people with diabetes. Diabetic Medicine, 23, 1332-1338.

Gazmararian, J. A., Williams, M. V., Peel, J., \& Baker, D. W. (2003). Health literacy and knowledge of chronic disease. Patient Education and Counseling, 51, 267-275.

Goran, M. I., \& Gower, B. A. (2001). Longitudinal study on pubertal insulin resistance. Diabetes, 50, 2444-2450.

Greening, L., Stoppelbein, L., Konishi, C., Jordan, S. S., \& Moll, G. (2006). Child routines and youths' adherence to treatment for Type 1 diabetes. Journal of Pediatric Psychology, 32, 437-447. 
Grey, M. (2011). Coping skills training for youths with Type 1 Diabetes. Diabetes Spectrum, 24, 70-75.

Grey, M., Boland, E. A., Davidson, M., Li, J., \& Tamborlane, W. (2000). Coping skills training for youth with diabetes mellitus has long-lasting effects on metabolic control and quality of life. The Journal of Pediatrics, 137, 107-113.

Grey, M., Boland, E.A., Davidson, M.,Yu, C., Sullivan-Bolyai, S., \& Tamborlane, W. V. (1998). Short-term effects of coping skills training as adjunct to intensive therapy in adolescents. Diabetes Care, 21, 902-908.

Grey, M., Cameron, M. E., Lipman, T. H., \& Thurber, F. W. (1995). Psychosocial status of children with diabetes in the first 2 years after diagnosis. Diabetes Care, 18, 1330-1336.

Grey, M., Whittemore, R., Jaser, S., Ambrosino, J., Lindemann, E., Liberti, L.,...Dziura, J. (2009). Effects of coping skills traning in school-age children with Type 1 diabetes. Research in Nursing \& Health, 32, 405-418.

Grey, M., Whittemore, R., Liberti, L., Delamater, A. M., Murphy, K., \& Faulkner, M. S. (2012). An comparison of two internet coping programs for adolescents with Type 1 diabetes. Contemporary Clinical Trials, 33, 769-776.

Grey, M., Whittemore, R., \& Tamborlane, W. (2002). Depression in Type 1 diabetes in children: Natural history and correlates. Journal of Psychosomatic Research, 53, 907-911.

Hains, A. A., Berlin, K. S., Davies, W. H., Smothers, M.K., Sato, A. F., \& Alemzadeh, R. (2007). Attributions of adolescents with Type 1 diabetes related to performing 
diabetes care around friends and peers: The moderating role of friend support. Journal of Pediatric Psychology, 32, 561-570.

Hanberger, L., Ludvigsson, J., \& Nordfeldt, S. (2009). Health-related quality of life in intensively treated young patients with type 1 diabetes. Pediatric Diabetes, 10 , 374-381.

Hanna, K. M., \& Guthrie, D. (2000). Adolescents' perceived benefits and barriers related to diabetes self-management-Part 1. Issues in Comprehensive Pediatric Nursing, $23,165-174$.

Hanna, K. M., \& Guthrie, D. (2000). Parents' perceived benefits and barriers related to diabetes self-management-Part 2. Issues in Comprehensive Pediatric Nursing, 23, 193-202.

Hanna, K. M., \& Guthrie, D. (2001). Parents' and adolescents' perceptions of helpful and nonhelpful support for adolescents' assumption of diabetes management responsibility. Issues in Comprehensive Pediatric Nursing, 24, 209-223.

Hesketh, K. D., Wake, M. A., \& Cameron, F. J. (2004). Health-related quality of life and metabolic control in children with Type 1 diabetes: A prospective cohort study. Diabetes Care, 27, 415-420.

Hoey, H., Aanstoot, H.-J., Chiarelli, F., Daneman, D., Danne, T., \& Dorchy, H. (2001). Good metabolic control is associated with better quality of life in 2,101 adolescents with type 1 diabetes. Diabetes Care, 24, 1923-1928. 
Hood, K., K., Huestis, S., Maher, A., Butler, D., Volkening, L., \& Laffel, L. M. B. (2006).Depressive symptoms in children and adolescents with Type 1 Diabetes. Diabetes Care 29, 1389-1391.

Individuals with Disabilities Education Act Amendments, 1997, Pub. L. No. 105-17, 111 Stat. 37 (1997).

La Greca, A. M., Auslander, W. F., Greco, P., Spetter, D., Fisher, E. B., \& Santiago, V. J. (1995). I get by with a little help from my family and friends: Adolescents' support for diabetes care. Journal of Pediatric Psychology, 20, 449-476.

Laffel, L. M. B., Connell, A., Vangsness, L., Goebel-Fabbri, A., Mansfield, A., \& Anderson, B. J. (2003). General quality of life in youth with type 1 diabetes: Relationship to patient management and diabetes-specific family conflict. Diabetes Care, 26, 3067-3073.

Lawson, M. L., Cohen, N., Richardson, C., Orrbine, E., \& Pham, B. (2005). A randomized trial of regular standardized telephone contact by a diabetes nurse educator in adolescents with poor diabetes control. Pediatric Diabetes, 6, 32-40.

Leonard, B. J., Garwick, A., Adwan, J. Z. (2005). Adolescents' perceptions of parental roles and involvement in diabetes management. Journal of Pediatric Nursing, 20, 405-414.

Littlefield, C. H., Craven, J. L., Rodin, G. M., Daneman, D., Murray, M. A., \& Rydall, A. C. (2002). Relationship of self-efficacy and bingeing to adherence to diabetes regimen among adolescents. Diabetes Care 15,90-94. 
Lorig, K. R., Sobel, D. S., Ritter, P. L., Laurent, D., \& Hobbs, M. (2003). Selfmanagement and education: History, definition, outcomes, and mechanisms. Annals of Behavioral Medicine, 26:1-7.

Miller, V. A. \& Drotar, D. (2003). Discrepancies between mother and adolescent perceptions of diabetes-related decision-making autonomy and their relationship to diabetes-conflict and adherence to treatment. Journal of Pediatric Psychology, $28,265-274$.

Moran, A., Jacobs, D. R., Steinberger, J., Hong, C.P., Prineas, R., \& Luipker, R (1999). Insulin resistance during puberty: results from clamp studies in 357 children. Diabetes, 48: 2039-2044.

Mulvaney, S. A., Rothman, R. L., Wallston, K. A., Lybarger, C., \& Dietrich, M. S. (2010). An internet-based program to improve self-management in adolescents with Type 1 diabetes. Diabetes Care, 33, 602-604.

Murphy, H. R., Wadham, C., Rayman, G., \& Skinner, C. T. (2007). Approaches to integrating paediatric diabetes care and structured education: experiences from the Families, Adolescents, and Children's Teamwork Study (FACTS). Diabetic Medicine, 24, 1261-1268.

Murphy, H. R., Wadham, C., Hassler-Hurst, J., Rayman, G., \& Skinner. (2012). Randomized trial of a diabetes self-management education and family teamwork intervention in adolescents with Type 1 diabetes. Diabetic Medicine, 29, e249$\mathrm{e} 254$. 
Nichols, S. J., \& Norris, S. L. (2002). A systematic literature review of the effectiveness of diabetes education of school personnel. The Diabetes Educator, 28, 405-414. doi: $10.1177 / 014572170202800310$.

Nicholas, D. B., Fellner, K. D., Frank, M., Small, M., Hetherington, R., Slater, R., \& Daneman, D. (2012). Evaluation of an on-line education and support intervention for adolescents with diabetes. Social Work in Health Care, 51, 815827.

Ott, J., Greening, L., Palardy, N., Holderby, A., \& DeBell, W. K. (2000). Self-efficacy as a mediator variable for adolescents' adherence to treatment for insulin-dependent diabetes mellitus. Children's Health Care, 29(1), 47-63.

Ryan, P., \& Sawin, K. J. (2009). The individual and family self-management theory: Background and perspectives on context, process, and outcomes. Nursing Outlook, 57, 217-225.

Schilling, L. S., Grey, M., \& Knafl, K. A. (2002). The concept of self-management of type 1 diabetes in children and adolescents: An evolutionary concept analysis. Journal of Advanced Nursing, 37, 87-99.

Schillinger, D., Grumbach, K., Piette, J., Wang, F., Osmond, D., Daher, C., \& al. (2002). Association of health literacy with diabetes outcomes. Journal of the American Medical Association, 288, 475-482.

Silverstein, J., Klingensmith, G., Copeland, K., Plotnick, L., Kaufman, F., \& Laffel, L. (2005). Care of children and adolescents with type 1 diabetes. Diabetes Care, 28(1), 186-212. 
Skinner, T. C., John, M., \& Hampson, S. E. (2000). Social support and personal models of diabetes as predictors of self-care and well-being: A longitudinal study of adolescents with diabetes. Journal of Pediatric Psychology, 25, 257-267.

Szadkowska, A., Pietrzak, I., Mianowska, B., Bodalska-Lipinska, Keenan, H. A., Toporowska-Kowalska...Bodalski, J. (2007). Insulin sensitivy in Type 1 diabetes children and adolescents. Diabetic Medicine, 25, 282-288.

Urbach, S. L., LaFranchi, S., Lambert, L., Lapidus, J. A., Daneman, D., \& Becker, T. M. (2005). Predictors of glucose control in children and adolescents with Type 1 diabetes mellitus. Pediatric Diabetes, 6:69-74.

Varni, J. W., Burwinkle, T. M., Jacobs, J. R., Gottschalk, M., Kaufman, F., \& Jones, K. L. (2003). The PedsQL in Type 1 and Type 2 diabetes: Reliability and validity of the Pediatric Quality of Life Inventory generic core scales and type 1 diabetes module. Diabetes Care, 26, 631-637.

Vocational Rehabilitation Act of 1973, Section 504, 29 U.S.C. § 794 (retrieved from http://www.epa.gov/ocr/docs/40p0007.pdf)

Wagner, V. M., Mueller-Goddeffroy, E., von Sengbusch, S., Hager, S., \& Thyen, U. (2005). Age, metabolic control and type of insulin regime influences healthrelated quality of life in children and adolescents with diabetes mellitus. European Journal of Pediatrics, 164, 491-496.

Weibe, D. J., Berg, C. A., Korbel, C., Palmer, D. L., Beveridge, R. M., Upchurch, R.,...Donaldson, D. L. (2005). Children's appraisals of maternal involvement in coping with diabetes: Enhancing our understanding of adherence, metabolic 
control, and quality of life across adolescence. Journal of Pediatric Psychology, $30,167-178$.

White, N. H., Cleary, P. A., Dahms, W., Goldstein, D., Malone, J., \& Tamborlane, W. (2001). Beneficial effects of intensive therapy of diabetes during adolescence: Outcomes after the conclusion of the Diabetes Control and Complications Trial (DCCT) Journal of Pediatrics, 139, 804-812.

Whittemore, R. \& Grey, M. (2002). The systematic development of nursing interventions. Journal of Nursing Scholarship, 34, 115-120.

Wysocki, T., Harris, M. A., Buckloh, L. M., Mertlich, D., Lochrie, A. S., Mauras, N., \& White, N. H. (2007). Randomized trial of behavioral family systems therapy for diabetes. Diabetes Care, 30, 555-560.

Wysocki, T., Harris, M. A., Buckloh, L. M., Mertlich, D., Lochrie, A. S., Taylor,...White, N. H. (2006). Effects of behavioral family systems therapy for diabetes on adolescents' family relationships, treatment adherence, and metabolic control. Journal of Pediatric Psychology, 31, 928-938. 


\title{
Chapter 3
}

Methodology

Managing Missing Data in a Correlational, Descriptive, Cross-sectional, Exploratory Study Using Survey Methods

\begin{abstract}
The problem of missing data in survey research is commonplace. This manuscript addresses empirical and theoretical approaches for selecting methods to manage missing data in survey research designs. Various approaches to managing missing data are examined. Newer methods including Multiple Imputation (MI) and Expectation Maximization (EM) are endorsed as the most appropriate methods for managing missing data within survey research studies. Recommended best practices are proposed for addressing missingness and an actual cross-sectional research study involving 103 parentadolescent dyads using survey methods is used to demonstrate a realistic example of how one nurse researcher managed missing data.
\end{abstract}

Key words: Missing data, analysis, survey methodology, multiple imputation 
A common circumstance associated with survey design research is dealing with a large number of characteristics and/or behaviors within data sets. A majority of survey research data sets have at least some missing data and predictably, data are missing on several variables for a number of cases. Even a small number of missing observations, though likely accounting for less statistical bias than larger proportions, can be problematic. To minimize the potential for negative repercussions related to bias, a plan for managing missing data needs to be carefully crafted at the outset of a study. The implications of not addressing missing data in the preliminary phases of a study are substantial because it can threaten both internal (statistical power) and external validity (generalizability of research findings) (Acock, 1997; McKnight, McKnight, Sidani, \& Figueredo, 2007; Schafer \& Graham, 2002). The purpose of this manuscript, therefore, is to review the various types of missing data, expand on approaches to managing missing data, and provide an exemplar for nurse researchers faced with the problem of missing data in survey research.

\section{Evaluating Missing Data}

\section{Assessing Quantity and Patterns of Missing Data}

It is important to first assess how much data are missing and consider why certain data are missing. There are three ways the amount of mis sing data can be assessed. The most common is to identify the number of participants in a study with incomplete data. A researcher can also include the number of missing responses on a given variable being analyzed or the number of missing responses in an entire data set. Each of these methods for interpreting missing data has implications with regard to statistical power. 
The volume of missing data is important to consider when determining the statistical method to use for handling missing data. How much missing data is acceptable? The obvious response is the smallest amount possible. Although acceptable rates for missing data have not been clearly determined, it is important to establish an appropriate rate/amount of missing data in a study. Theodor and Gatchel (2008) suggested that a "general rule of thumb" for data missing in a random pattern should be less than 5\% for each individual variable within a data set. Sterner (2011) suggested that response rates of less than $80 \%$ for survey variables should be considered concerning. Nonresponse rates for the overall study along with nonresponse rates for each variable should be reported with inclusion of the rationale for missing data.

Tabachnick and Fidell (2007) advised that the pattern of missing data is more important than the amount of missing. Details to consider include: Is there consistency or predictability in participants missing data? Is there a detectable pattern present? It is the researcher's responsibility to provide a rationale for the rate of missing data and any patterns of missing data in a study. One should consider all the unique factors associated with a study that could ultimately affect missing data, which is also referred to as missingness. If there is any missing data within a study for any reason, the researcher must consider whether the data collected represents the variable under study and whether the data reliably reflects the study outcome.

\section{Classifying Missing Data}

The dilemma of bias within survey research studies raises concerns regarding not only how much missing data is acceptable in a study but whether data are missing at 
random or not missing at random (Little \& Rubin, 2002). Whenever there is missing data, it is important to try to understand why certain responses are missing. Identifying plausible reasons why data are missing can help with selecting the most optimal method for addressing this problem. A classification system that describes relationships between measured variables and the probability of missing data was first proposed by Rubin (1976), further expanded upon by Little and Rubin (2002), and is still widely used today.

Missing value mechanisms. Rubin (1976) identified three unique kinds of 'mechanisms' generating missing values and categorized them into the following subtypes: (a) data missing completely at random (MCAR), (b) data missing at random (MAR), and (c) data missing not at random (MNAR). Each mechanism can be thought of as an assumption that prompts the operation of different missing data techniques.

Missing completely at random (MCAR). MCAR is a situation where there is no systematic reason why data are missing in a study; missing data are determined to occur completely at random. The missing values within a case could be missing because a participant relocates, or misses one of the survey administrations because of a scheduling difficulty or simply skips over an item(s) in a survey. MCAR analyses generate unbiased parameter estimates; the estimates approach population values (McKnight et al., 2007). In Verchota's (2014) cross-sectional, correlational study, 103 parent-teen dyads were surveyed on a variety of variables hypothesized to be associated with self-management in adolescents with Type 1 Diabetes Mellitus (T1DM). A situation occurred where two full pages of two of the parent paper and pencil surveys were left entirely blank-never filled 
out by the participant, likely related to human error. This is a classic example of data MCAR.

Missing at random (MAR). When data are MAR, there is a random pattern of missing data demonstrated with specific subgroups (Sterner, 2011). The MAR mechanism is conditional; MAR happens when missing data can be correlated with or are dependent upon some other observed participant characteristic. Missingness with MAR data can be considered more a function of the characteristics of the participant than a function of the missing values (Sinharay, Stern, \& Russell, 2001). An example of MAR from Verchota's (2014) study was derived from the PedsQL Diabetes Module (Varni, Limbers, \& Burwinkle, 2007) designed to address unique problems associated with T1DM. Item 2 of the instrument states: "I feel thirsty" and provides five responses to choose from: "never" to "almost always". Nine adolescents $(8.7 \%)$ left this item blank. After a careful review, the researchers found that a majority of respondents leaving this item blank also had a hemoglobin A1c between 9.4 and 11\%, clearly indicating poor diabetes control and an expected symptom of thirst. It is not clear why study participants did not answer this particular question (participants were told they could skip any questions they did not want to answer). Perhaps some found this question threatening; perhaps, others did not want to reveal symptoms that would point towards poor diabetes control. To the extent that missingness can be correlated with other variables in the analysis, data are considered MAR and unbiased parameter estimates can be generated.

Missing not at random. MNAR occurs when there is a systematic reason why data are missing. There is not an equal chance that values are missing across a variable. 
The missing data are in some way related to the value one is attempting to identify (McKnight et al., 2007). This mechanism is much more problematic for researchers because participants have decided not to respond to one or more items or questions and it is not always apparent to the researcher why this has occurred. MNAR creates parameter estimates that are biased and therefore not reliable. Often a researcher cannot classify missing data into just one mechanism; missing data in any given study are likely MCAR, MAR or MNAR (McKnight et al., 2007).

Ignorability. If a researcher fails to confirm MCAR, the mechanism is determined not to be MCAR and is either MAR or MNAR (Little \& Rubin, 2002). It is not possible to statistically distinguish between MAR and MNAR. In order to establish whether missing data are MAR or MNAR, the concept of ignorability is important. According to Rubin (1976), missing data are ignorable when the mechanism creating the missing data is related to information that is known; it can be modeled with observed data within a data matrix. Ignorable mechanisms are easier to manage because their effect can be determined by statistical modeling. A mechanism can be considered ignorable when data are MAR and the parameters that govern the missing data are not related to the parameters to be estimated (Allison, 2002).

Missing values are considered nonignorable when there appears to be a systematic, nonrandom process underlying the missingness, yet the reason for this missingness is not understood. It doesn't appear to the researcher that there are any other data in the matrix that could model the way this particular data are missing. An analysis of parameter estimates and subsequent statistical conclusions could potentially be biased, 
thus affecting the generalizability of research findings (Musil, Warner, Yobas, \& Jones, 2002). Conclusions based on data determined to be MNAR and nonignorable can result in "potentially dangerous" outcomes because of the level of bias that can be exerted on the statistical analysis (McKnight et al., 2007). Therefore, the vast majority of missing data handling techniques require missing data to be of the ignorable type.

Categories of missing data. McKnight et al. (2007) asserted that the process a researcher undergoes to manage missing data should not only entail identifying key mechanisms of missingness but also consider other reasons why data might be missing and how missing data could potentially impact study results. Three general categories generate the problem of missing data: study participants, study design, and the interaction between the study participants and the design (McKnight et al.). There are numerous reasons why participant data can be missing; some participants might choose not to respond to an item as hypothesized earlier, they could miss an item, select more than one item, an item response might be illegible to the researcher, or a computer/ software programs malfunctions and data are lost. Longitudinal studies can be even more problematic with regard to missing data; researchers find that participants either relocate or drop out of a study or are not able to respond at one or more times when a survey response is planned. When eligible participants do not participate in a study for any reason, the missing data are referred to as survey nonresponse. This is in contrast to when a particular continuous and/or categorical variable(s) are missing. This is referred to as item nonresponse (Little \& Rubin, 2002). 


\section{The Gap Between Recommendations and Reality}

A gap remains between methods currently recommended for handling missing data and how researchers manage and report missing data despite significant advancements made by statisticians over the last several decades regarding how to deal with missing data. Explicit recommendations from the APA (2010) have been issued for researchers to report percentages or frequency of missing data along with empirical evidence and/or theoretical rationale for why data are missing in a study. This gap persists for the following reasons: (a) newer approaches for managing missing data are more difficult to grasp because of their technical nature when compared to older methods where missing data meant excluding cases or merely replacing missing values with a mean (Beraldi \& Enders, 2010); (b) statistical analysis software packages include outdated procedures for managing missing data as the default option, sending a misguided message to nurse researchers about how missing data should be handled; and (c) published manuscripts often fail to include details regarding missing data so the management of missing data remains unclear.

\section{Approaches to Managing Missing Data}

\section{Traditional Missing Data Techniques}

Early methods for handling missing data used in nursing research studies encompassed deletion, direct estimation, and single imputation methods (often referred to mean substitution). Deletion methods include both listwise and pairwise deletions where cases are discarded during the analysis if they contain missing data. With listwise deletion (or "complete case analysis"), if a case has any missing value(s) for any variable, 
the case is deleted from the data analysis. Pairwise deletion (or "available case analysis") involves only deleting specific variable cell values when a particular variable is required in an analysis and has a missing value, yet the case will exist in all other situations. Many statistical software packages default to these approaches. The direct estimation process uses all available data, including variables where there are missing data to construct parameter estimates and standard errors.

Imputation methods include single imputation (such as mean imputation, hot-deck imputation (HD), last value carried forward (LVCF) method, regression method, etc.) and multiple imputation (MI). Single imputation is considered the most widely used estimation technique (Buhi, Goodson, \& Neilands, 2008). The central idea of imputation involves substituting a rational estimate (imputation) for each missing value and then conducting an analysis with essentially a complete set of data (Allison, 2002). This approach entails replacing missing values with a constant replacement; in mean imputation, the sample mean (arithmetic mean or estimated population mean) of a variable replaces any missing data for that variable. With HD imputation, missing values are replaced with values taken from matching respondents (i.e., someone in the study who shares the same pattern of responses). LVCF entails using the last observed value to fill in missing values at subsequent points in a longitudinal study. Regression method (predicted mean imputation) entails using an ordinary least-square multiple regression method to impute the predicted mean. In this method, a multiple regression equation based on complete case data is developed for a given variable with missing values, and treats it as the outcome using all other relevant variables as predictors (Acock, 2005). 
Missing values are imputed using predicted values from other corresponding variables using the estimated multiple regression model. Although single imputation methods offer a quick and easy method to increase the sample size back to its original size, a word of caution is in order. The variances and covariances that result from imputing a single value will be biased downward, yielding decreased correlations among variables (McKnight et al., 2007). These methods often result in biased estimates, incorrect standard errors, or both (Little \& Rubin, 2002).

\section{Newer Data Imputation Methods}

Available to the nurse researcher today are statistical software programs with greater computing capabilities that allow the application of more technically refined and developed statistical analysis tools to manage missing data. Two newer methods currently recommended for managing missing data include Multiple Imputation (MI) methods and Maximum Likelihood (ML) techniques (Allison, 2002; Schafer \& Graham, 2002). These methods are characterized in the literature as augmentation or model-based procedures and considered superior to the methods described earlier, primarily bec ause they mitigate the pitfalls of the more traditional techniques and provide unbiased estimates when data are understood to be missing in a random fashion (either MAR or MCAR).

Multiple imputation (MI). MI is a more sophisticated missing data technique where missing values are replaced with two or more imputed values, usually from three to $10 . \mathrm{MI}$ is the recommended approach for handling missing data with large sample sizes, an example being census data. MI is less sensitive with regard to the missing data 
mechanism (it assumes that missing data are MAR) and estimates the influence of the missing data on parameter estimation, something other methods do not include. The MI method is a robust method of missing data analysis and is highly regarded (Allison, 2002; Schafer \& Graham, 2002). MI amends the lowered variance problem seen with the single imputation method, adjusting standard errors upward thus reducing the likelihood of a Type 1 error (McKnight et al., 2007). A strength of MI is that once the imputed data sets have been created, they can be used in any type of analysis, from descriptive statistics to complex multivariate analyses.

Scholars of missing data analytic procedures warn that although MI is becoming increasingly available, it is computationally complex and thus remains an approach designed for more experienced data analysts. McKnight et al. (2007) suggested MI is "somewhat beyond the reach of novice analyst" (p. 211). It has been suggested that augmentation methods such as Expectation Maximization, a model-based approach, can be more efficient than MI because no simulation is involved and parameters can be calculated directly from incomplete data (McKnight et al., 2007).

Maximum likelihood (ML). ML is a popular model-based approach that was originally designed for estimating parameters in structural equation models and hierarchical linear models and later adopted for use with missing data. The basic tenant of $\mathrm{ML}$ is to select estimates that contain values that maximize the probability of obtaining the observed data. This is done using a formula that estimates the likelihood of the data as a function of both the data and the unknown parameters. When missing data are 
determined to be ignorable, estimating probabilities over all possible values of the missing data and summing them will provide the ML estimation.

Expectation maximization (EM). EM is a method for obtaining ML estimates. The EM procedure provides parameter estimates for the data set, both observed and missing values. EM considers observed data, missing data, relationships between observed data and underlying assumptions in order to be able to estimate parameters. EM is classified as a model-based procedure, having the additional advantage of being able to construct robust parameter estimates when there is missing data (McKnight et al., 2007). EM does not use an imputation procedure; instead, EM uses observed data along with additional information provided by a probability model to create a missing data correlation matrix through two unique steps. In the first step, the Expectation (E) step, parameter estimates are based on all complete data sets. In the Maximization (M) step, the expected values are substituted for the missing data by E step values and ML estimation is computed. This procedure is repeated in an iterative process until convergence is eventually achieved and the resulting data are filled in for the missing data and saved in the data set.

\section{Practical Steps for Handling Missing Data in Survey Research Designs: An}

\section{Exemplar}

The following section includes an example taken from an actual survey research study by Verchota (2014) where a relatively large number of characteristics and/or behaviors of study participants (adolescent and parent dyads) was collected. Predictably, there was evidence of some missing data present within several of the data sets. The first 
step was to understand more about the volume of missing data from each item in the survey. The "Missing Value Analysis" procedure in SPSS can help describe the pattern of missing data, estimated means, standard deviations, and covariances for listwise, pairwise, regression and EM, and can impute missing values with estimated values using EM methodology. A majority of items had less than $2 \%$ missing data. Missing data accounted for less than $5 \%$ of each of the subscales under investigation except for several items in the Diabetes QOL variable.

The next step involved determining if there was a pattern to any of the missing data and assess where missing values were located. This was followed by a more indepth analysis to determine whether there were pairs of variables that had values missing across cases. An example of this was the topic of wearing an identification bracelet. The Self-Efficacy Scale (Grossman, Brink, \& Hauser, 1987) included the statement: "I carry something with me that says I have diabetes." Nine percent of participants left this item blank in the study. When completing the PedsQL Diabetes Module, Treatment subscale (Varni et al., 2003), 14.4\% of the adolescents left the following item blank: "It is hard for me to wear my ID bracelet." In the parent's corresponding rating of their son or daughter's diabetes quality of life (PedsQL Parent Version, Varni et al., 2007), 6.7\% of the parents left the following item blank: "It is hard for my teen to wear his/her ID bracelet." It is not likely this is an entirely random occurrence; in this case it is more plausible that adolescent participants chose not to respond to the statement because they don't abide by this recommendation and consequently the basis for the missing data. It is 
postulated that wearing an ID bracelet could draw attention to the fact that the adolescent has diabetes, thus setting them apart from peers.

The next step involved testing the hypothesis that missing data are MCAR by running Little's MCAR test on variables with missing data within SPSS. Little's (1988) MCAR test contrasts observed variable means for each pattern of missing data with expected population means and a weighted squared deviation. If data are MCAR, each subsample corresponding with a specific pattern of data should produce the same means for each variable as those computed for the entire set. The inferred hypothesis is that missing values are missing in a random way. Little's method takes the weighted sums of squared deviations from the maximum likelihood (the expected population mean) estimates by comparing the sum with the chi-square table (the degrees of freedom is equal to the sum of the number of variables for each pattern minus the total number of variables). If there are differences between missing and non-missing cases for the observed data, the chi-square test is considered significant and the researcher concludes that the missing data are not MCAR (Little, 1988). There is not a specific table for Little's MCAR test within SPSS; instead Little's MCAR test results are included as a footnote in each the Means, Covariance and Correlations tables in Missing Values Analysis as depicted in Table 2. For the predictor variables under study with missing data, Little's MCAR test indicated the findings were not significant. In each case the null hypothesis was accepted, indicating missing data are likely MCAR.

Once the mechanism for missingness was determined, the EM method was used to impute values for missing items on each predictor variable (see SPSS syntax example, 
Figure 4). Missing values were replaced by imputed values and saved into a new data file allowing for further analysis.

The instructions for scoring the PedsQL recommend that if more than $50 \%$ of the items in a scale are missing, the scale scores should not be computed. All scales/subscales included in the study with missing data comprised less than $50 \%$ of the items. When less than $50 \%$ of items in the scale are missing, the recommendation is to calculate mean scores by Dimension and then sum all the items over the number of items answered on all the scales. A "PedsQL Total Score" was generated in this manner and then recalculated using general mean substitution measures and finally recalculated using the EM methodology. In the final analysis, there was a trivial difference between the mean substitution score and the EM obtained scores for the PedsQL (see Table 3).

Finally, a descriptive analysis was undertaken comparing listwise, mean substitution, and EM methods for each predictor variable containing missing data (Table 4). Because only a very small percent of the data were missing in the data set in this example, it was difficult to recognize any significant difference between different imputation methods. For this reason, one would also not expect a difference between the results with imputation and without imputation.

\section{Recommendations}

There is not an easy solution for the problem of missing data. When data are MAR, either ML or MI are better options for managing missing data than traditional methods. If this assumption is accurate, resulting estimates (i.e., regression coefficients 
and standard errors) are considered unbiased with no loss of power and comprised of accurate standard errors using either method (Allison, 2002).

For nurse researchers conducting survey research studies, ML is recommended over MI for a number of reasons. ML is parsimonious; the analysis is handled with a single model for understanding the distribution of variables with missing data (Allison, 2002). ML is considered a convenient and efficient method for analyzing missing data and is currently available on many software programs (i.e., Amos). Recall that ML does not impute data; instead ML uses each cases full incomplete data set to compute ML estimates. Therefore, the ML estimate of a parameter is most likely to result in data that are observed (Allison). A shortcoming of ML is that as a missing data methodology it is limited to linear and log-linear models including linear regression, structural equations with latent variables, factor analysis and simultaneous equations (for a detailed example of ML, see Allison, 2002, p 27). With linear models, data are required to be normally distributed (Allison). ML is also generally recommended when it is anticipated that participants will drop out at various points in time and not return to a study (referred to as a monotonic pattern where data are missing on a particular variable and subsequently missing for variables that come later in the order).

MI on the other hand can be used on almost any kind of data or model and can be run on conventional software. A caveat with MI: It can be cumbersome and is prone to error. Because random draws are a key part of the process in MI, MI creates several copies of a data set, with different estimates obtained every time an analysis is run. By 
imputing more data sets one can decrease the variability, yet it is not known how many imputations are considered adequate (Allison, 2002).

Missing data in longitudinal studies presents unique challenges for the nurse researcher. Hogan, Roy, and Korkontzelou (2004) provide an excellent tutorial regarding models and methods for managing missing data (primarily drop-outs) in longitudinal designs.

\section{Summary}

Missing data can threaten the reliability, validity, and generalizability of conclusions made in a research study. It is therefore important to plan for missing data, consider the types of missing data present in a study, and identify ways to reduce their negative effects. Statisticians have made significant progress with regard to handling missing data. Newer analytical strategies can facilitate maximizing the use of all data collected in a research study. Little's MCAR test can help to determine if data are MCAR. The two step EM procedure applied to study variables with missing data (after determining that missing data are of the ignorable type) is an effective method for handling the problem of missing data estimates.

Imputation can increase the accuracy of the parameter estimation and therefore increase the power of the statistical analysis. The data set used for this exemplar did not contain missing clinical data; missing data were derived from study scales. Each scale had good reliability; therefore, one would expect a mean to function well. When there is a small amount of missing data, as was generally the case for this study, differences between the various recommended methods for handling missing were minimal. 
Irrespective of the method researchers use to address the issue of missing data, there is currently no method available that tests the robustness of assumptions made about missing data. This reinforces the importance of addressing the need for a sound study design at the outset; allowing the nurse researcher to carry out the study in a manner that limits missing data as much as possible. 


\section{Reference List}

Acock, A. (1997). Working with missing data. Journal of Child Psychology, 21, 407-412.

Acock, A. (2005). Working with missing values. Journal of Marriage and Family, 67, 1012-1028.

Allison, P. D. (2002). Missing data. Thousand Oaks, CA: Sage.

American Psychological Association (2010). Publication manual of the APA.

Washington, DC: American Psychological Association.

Beraldi, A. N., \& Enders, C. K. (2010). An introduction to modern missing data analysis. Journal of School Psychology, 48(1), 5-37.

Buhi, E. R., Goodson, P., \& Neilands, T. B. (2008). Out of sight, not out of mind: Strategies for handling missing data. Am J Health Behavior, 32(1), 83-92.

Grossman, H. Y., Brink, S., \& Hauser, S. T. (1987). Self-efficacy in girls and boys with insulin-depe ndent diabetes mellitus. Diabetes Care, 10,324-329.

Hogan, J. W., Roy, J., \& Korkontzelou, C. (2004). Tutorial in biostatistics: Handling drop-out in longitudinal studies. Statistics in Medicine, 23, 1455-1497.

Little, R. J. (1988). A test of missing completely at random for multivariate data with missing values. Journal of the American Statistical Association, 83(404), 11981202.

Little, R. J., \& Rubin, D. B. (2002). Statistical analysis with missing data. New Jersey: John Wiley \& Sons, Inc.

McKnight, P. E., McKnight, K. M., Sidani, S., \& Figueredo, A. J. (2007). Missing Data: A Gentle Introduction. New York: The Guilford Press. 
Musil, C. M., Warner, C. B., Yobas, P. K., \& Jones, S. L. (2002). A comparison of imputation techniques for handling missing data. Western Journal of Nursing Research, 24(7), 815-829.

Rubin, D. B. (1976). Inference and missing data. Biometrika, 63, 581-592.

Schafer, J. L., \& Graham, J. W. (2002). Missing data: Our view of the state of the art. Psychological Methods, 7(2), 147-177.

Sinharay, S., Stern, H. S., \& Russell, D. (2001). The use of multiple imputation for the analysis of missing data. Psychological Methods, 6(4), 317-329.

Sterner, W. R. (2011). What is missing in counseling research? Reporting missing data. Journal of Counseling \& Development, 89, 56-62.

Tabachnick, B. G., \& Fidell, L. S. (2007). Using Multivariate Statistics (Fifth ed ed.). Boston: Pearson.

Theodore, B. R., \& Gatchel, R. J. (2008). Management and interpretation of data obtained from clinical trials in pain management. Pain Practice, 8(6), 461-472.

Varni, J. W., Burwinkle, T. M., Jacobs, J. R., Gottschalk, M., Kaufman, F., \& Jones, K. L. (2003). The PedsQL in Type 1 and Type 2 diabetes: Reliability and validity of the Pediatric Quality of Life Inventory generic core scales and type 1 diabetes module. Diabetes Care, 26, 631-637.

Varni, J. W., Limbers, C. A., \& Burwinkle, T. M. (2007). Parent proxy-report of their children's health related quality of life: An analysis of 13,878 parents' reliability and validity across age subgroups using the PedsQL 4.0 generic core scales. Health and Quality of Life Outcomes, 5, 1-10. 
Verchota, G. M. (2014). Testing components of a self-management theory in adolescents with Type 1 Diabetes Mellitus (Unpublished Dissertation). University of Wisconsin, Milwaukee. 


\title{
Chapter 4
}

Testing Components of a Self-management Theory

\begin{abstract}
Background: The role of self-management (SM) in adolescents with Type 1 Diabetes Mellitus (T1DM) is not well understood. Objectives: Examine the relationship of key Individual and Family Self-management Theory (IFSMT) context and process variables on proximal (self-management (SM) behaviors) and distal (hemoglobin A1c [HgbA1c] and diabetes-specific health-related quality of life [DQOL]) outcomes in adolescents with T1DM. Methods: A correlational, cross-sectional study was undertaken to identify factors contributing to effective SM in adolescents with T1DM and further examine potential direct and mediating relationships that exist between context, process, and outcome variables delineated in the IFSMT. Participants included 103 adolescent-parent dyads (adolescents aged 12-17 years with T1DM) from a Midwest outpatient diabetes clinic. The dyads each completed a self-report survey including instruments intended to measure context, process, and outcome variables from the IFSMT. Results: Using hierarchical multiple regression, context (depressive symptoms) and process (communication) variables explained $37 \%$ of the variance in SMB. Regimen complexity explained $11 \%$ of the variance in metabolic control. Only regimen complexity was significant at Step 1. Neither of the regression steps adding process variables or SMB was significant. For the DQOL outcome, context (regimen complexity and depressive symptoms) explained $26 \%$ of the variance at Step 1, an additional $9 \%$ of the variance was explained when process (SE and communication) variables were added at Step 2 and a
\end{abstract}


total of $52 \%$ of the variance was explained when SMB were added at Step 3. In the final model, three variables were significant: depressive symptoms, SE, and SMB. The criteria for mediation were not met. Discussion: IFSMT can serve as a cogent theory for understanding key concepts, processes, and outcomes essential to SM in adolescents and families dealing with T1DM.

Keywords: Type 1 Diabetes Mellitus, adolescence, self-efficacy, communication, theory development 
Each year more than 15,000 youth are diagnosed with Type 1 Diabetes Mellitus (T1DM) in the United States (CDC, 2011). Recent epidemiological data indicate the most common age of onset of T1DM is from 10 to 14 years (Karvonen et al., 2000) with the incidence of T1DM increasing by $3 \%$ per year (Moltchanova, Schreier, Lammi, \& Karvonen, 2009). Individuals with T1DM are at increasing risk for developing chronic complications including retinopathy, renal disease and neuropathy (Diabetes Control and Complications Trial [DCCT],1994).

T1DM has been described as one of the most behaviorally and psychologically demanding of the chronic diseases related to the extensive daily requirements associated with self-management (SM). The developmental period of adolescence adds a particularly challenging dimension for youth with diabetes. Research has consistently demonstrated that children with T1DM experience a decline in metabolic control as they enter adolescence (Jacobson et al., 1994; Mortensen et al., 1992) and poor metabolic control often persists throughout adolescence (Amiel et al., 1986; Blethen et al., 1981). During adolescence, increasing levels of responsibility for SM are transferred from parents to adolescent. Until recently, there were no instruments addressing SM in adolescents with T1DM that were congruent with the current understanding of this concept. The purpose of this study was to further understand the process of SM in adolescents with T1DM and their families through the lens of the Individual and Family Self-management Theory (IFSMT). 


\section{Theoretical Frame work}

IFSMT, a middle range theory, seeks to further explain the impact of various context and process variables on health outcomes (Ryan \& Sawin, 2009). Smith and Liehr (2003) observed that middle range theories are "developed and grow at the intersection of practice and research to provide guidance for everyday practice and scholarly research rooted in the discipline of nursing" (p. xi). IFSMT was selected as the guiding framework for understanding SM from the context of adolescents living with T1DM. SM is conceptualized as:

A process in which individuals and families use knowledge and beliefs, selfregulation skills and abilities, and social facilitation to achieve health-related outcomes. Self-management takes place in the context of risk and protective factors specific to a health condition, physical and social environment and individual and family. Self-management is applicable to chronic conditions as well as health promotion" (UWM SMSC, 2011; Ryan and Sawin; 2009, Ryan 2009).

Outcomes in the IFSMT are understood to be either proximal or distal; success in attaining proximal or short-term outcomes leads to the achievement of distal outcomes. Proximal outcomes embody SMB. Distal outcomes in the IFSMT are organized into three categories: health status, quality of life, and health costs (Ryan \& Sawin, 2009). IFSMT proposes that relationships exist between context and process variables impacting both proximal and distal outcomes for individuals and families as they assume various roles and responsibilities integral to successful chronic disease management (Ryan \& Sawin, 2009) (see Figure 1).

By enhancing our understanding of how these key variables affect SMB and how SMB in turn can affect health outcomes, IFMST served as a cogent model for expanding 
upon the current science of diabetes SM. Applying key concepts and propositions from the IFSMT to a subset of adolescents with T1DM in this study adds further clarity in understanding how various context and process variables are related to clinical outcomes.

This study further examined the phenomenon of SM as conceptualized by Ryan and Sawin (2009) in adolescents with T1DM and factors that contributed to SM. Three research questions were explored:

- What is the relationship of key IFSMT context (e.g. regimen complexity, age, gender, depressive symptoms, and parent perceived life difficulty) and process variables (knowledge, self-efficacy, goal setting, problem solving, communication, collaboration, and autonomy support) on the IFSMT proximal outcome (SMB)?

- What is the relationship of key IFSMT context variables (e.g. regimen complexity, age, gender, depressive symptoms, and parent perceived life difficulty), process variables (knowledge, self-efficacy, goal setting, problem solving, communication, collaboration, and autonomy support), and the proximal outcome (SMB) on distal outcomes (HgbA1c and DQOL)?

- Does SM (as a proximal outcome) mediate the relationship of select process variables on two distal outcomes: metabolic control and DQOL?

\section{Methods}

This correlational, cross-sectional study included adolescents 12-17 years of age and their parents from a large Midwestern diabetes specially care clinic. Criteria for 
inclusion in the study: (a) diagnosis of T1DM for at least one year; (b) a consenting parent or guardian, (c) able to read English and (d) without developmental disabilities or major psychiatric symptomology. A total sample of 115 adolescent-parent dyads was assented/consented and 106 dyad surveys were returned (92\% return rate). Of the surveys returned, 3 were determined to be ineligible (two adolescents had Type 2 DM and 1 adolescent had a co-morbid life threatening illness) resulting in a sample of 103 adolescent-parent dyads.

\section{Procedures}

Approval for the study was obtained from the relevant Institutional Review Boards. Families were recruited through two mechanisms: First, families in the Pediatric Diabetes Behavioral Health Cohort Registry who had previously indicated an interest in participating in research studies were contacted by telephone regarding the study and to learn if they were interested in participating in the survey. Second, the researcher or research assistant approached families at a diabetes clinic visit. If potential participants expressed interest, the study was explained in depth by the researcher and consent/assent obtained. All participants were offered two options for completing the survey: paper and pencil or Internet based. Seven dyads completed the Internet version with the remainder completing the paper and pencil survey. Study materials were identical for both participation methods. In the paper and pencil group, two sets of surveys were provided to participants; one set included measures addressed to the adolescent to complete and the other set included parent materials. Adolescents and parents were encouraged to complete the surveys independently, to contact the investigator if they had any questions or 
concerns about the study, and to return the surveys in separate pre-labeled stamped mailing envelopes. Each participant received a $\$ 25.00$ gift card upon completion of the survey. Per study protocol, the PI calculated the results of the depressive symptoms scale for each returned survey within 24 hours of receiving the survey. Three adolescents scored above established criteria on a standardized depressive symptoms instrument, were contacted by telephone by the PI to discuss findings and referred to a counselor for further evaluation and management.

\section{Measures}

Data were collected using the medical record and a series of survey instruments and are presented here in the order of IFSMT categories: context, process and outcomes factors. Internal reliability for all scale scores are reported in Tables 5 and 6 . Except for parent education, parent autonomy support and parent perceptions surrounding T1DM management, reported variables are from the adolescent.

Context factors. Data addressing condition-specific variables, individual and family variables, and physical and social environment data investigated in the study were collected using the Demographic and Clinical Information Form (DCIF) and instruments described herein.

Condition-specific factors. The DCIF was an investigator created measure used to record information abstracted from medical records. Condition specific factors included disease duration (years, months since diagnosis) and complexity of diabetes management. The latter was measured by a Complexity Index and remission status. Complexity Index: Multiple Daily injections $=1$ or Continuous Subcutaneous Insulin 
Infusion $=2$ plus Conventional regimen $=1$ or Intensive regimen $=2$; potential score range: $2-4$; higher scores indicate increasing complexity. Remission status was indicated with either a No $=0$ or Yes $=1$. Adolescents were categorized as in remission if the HgbA1C + [4xinsulin dose (units per kilogram per $24 \mathrm{~h}$ )] was less than 9 (Mortensen et al, 2009).

Individual \& family factors. Demographics included subject's age, gender, and race. Parent's education level and family structure was obtained. Education level was collected from the parent report of the study questionnaire (attended/completed high school, attended college/formalize training, completed college/formalized training). Family structure was obtained from the DCIF (lives with parents, one parent, or other arrangement).

Depressive symptoms were measured using the 8-item PROMIS depressive symptoms instrument (PROMIS Ped SF v 1.0), normed for an adolescent population, which focuses on negative mood (sadness), loss of capacity to experience pleasure (anhedonia), negative views of the self (worthlessness and low self-esteem), and negative social cognition (interpersonal alienation and loneliness). The PROMIS is available as an 8-item or 28-item version. Olino et al. (2013) compared the Beck Depression Instrument, Center for Epidemiologic Studies-Depression (CED-D) scale and 2 versions of the PROMIS and found the PROMIS-Depression scale more robust, providing information over the greatest range of severity of symptoms with the highest measurement of precision when compared with the other instruments (indicated with both the short form and 28-item instrument (Olino et al.). Items use a 7-day recall period and 5-point 
response option (never, almost never, sometimes, often, almost always). The internal reliability coefficient was reported at 0.85 (Irwin et al., 2010).

Parent perception of T1DM management was measured with 3 of the 5 Family Management Measure subscales (Knafl et al., 2009). The 30 items were scored from 1 to 5, ("strongly disagree" to "strongly agree") and included Condition Management Ability (CM-A), Condition Management Effort (CM-E), and Family Life Difficulty (FLD). Higher CM-A scores indicate greater family manageability of T1DM, higher CM-E scores greater effort/work to manage T1DM and higher FL-D scores greater life difficulty in managing T1DM. Internal consistency reliability ranged from 0.72-0.91 and test-retest reliability, 0.71-0.94 (Knafl et al.).

Physical and social environment factors. The DCIF was used to capture residence (rural vs urban based on U.S. census data), access to health care (distance from clinic in miles) and utilization of health care. Utilization encompassed a combined score; the number of diabetes clinic visits plus ER visits/hospitalizations related to diabetes.

Process factors. Data addressing process variables were collected from adolescents using the instruments described below. Reliability coefficients from current study are included in Tables 5 and 6.

Knowledge and beliefs. Diabetes-specific knowledge was measured with the adolescent versions of the "Survey of Diabetes Understanding" (Butler et al., 2008). The 13-item instrument is designed to measure knowledge of key tasks associated with diabetes SM and is concentrated on issues surrounding blood glucose monitoring (BGM), interpreting data obtained from BGM and HgbA1c results. Respondents include: "agree, 
disagree, or unsure". One point is given for each correct answer, one point is subtracted for each incorrect response, and no points are given for an "unsure" response. Scores range from +13 to -13 . Standard or cut scores were not included in the reference by Butler et al. A standard was developed by judgmental method after review of the instrument by a Master's prepared Certified Diabetes Educator (CDC). A cut score of $70 \%$ (9 items) on the 13-item instrument was recommended.

Self-efficacy was measured with the 24-item diabetes-specific subscale from the Self-Efficacy for Diabetes Scale (SEDS) (Grossman et al., 1987). Respondents rate their degree of confidence on a 6-point scale ranging from 0 ("very sure I cannot") to 6 ("very sure I can") and possible scores range from 0 - 96. Reliability coefficients were 0.88 0.92 (Grossman et al., 1987; Chui, 2005).

Self-regulation. The Self-Management of Diabetes in Adolescents (SMOD-A), a self-report instrument created specifically for adolescents, comprised of 5 subscales (Collaboration with Parents, Diabetes Care Activities, Problem Solving, Diabetes Communication, Goals) was used to measure 2 self-regulation variables (Problem Solving and Goals), 2 social facilitation variables (Collaboration with Parents and Diabetes Communication) and SMB (Schilling et al, 2009). Higher scores are indicative of a higher amount of the concept being measured. Content validity of the SMOD-A and its subscales was assessed by three expert panels and reported as 0.93 . The alpha reliability estimates ranged from .71 to .85 . Stability at 2-weeks and 3 months was $>.70$ for each subscale except for goals (.60 at 2 weeks and .59 at 3 months) and diabetes communication (.69 at 2-weeks) (Schilling et al.). 
Goal setting was measured using the Goals subscale from the SMOD-A which delineates $7 \mathrm{SMB}$ goals important to the adolescent. Instructions include identifying if and how frequently each given item is a goal for the adolescent. Scores range from 0 to 21.

Planning and action was measured using the Diabetes Problem Solving Subscale of the SMOD-A. Included in the subscale are 7-items that address how the adolescent goes about making decisions regarding insulin, exercise, food intake, and blood sugars that are outside of target range (Schilling et al., 2009, p. 234). Scores range from 0 to 21.

Social facilitation. Social influence was an adolescent measure using the SMODA Diabetes Communication subscale. This 10-item subscale addresses the extent and frequency with which the adolescent communicates with parents, friends, and health care providers about diabetes related concerns; e.g., who they talk to if something is bothering them about having diabetes and revealing they have T1DM with peers. Scores can range from $0-30$.

Negotiated collaboration was measured using the SMOD-A Collaboration with Parents subscale which includes 13-items that address how often parents are involved in their teen's diabetes management. Details on scale items include whether the adolescent advises parents when blood sugars are outside target range, whether parents check glucose meter readings and help with deciding insulin dose and meal planning. Scores can range from 0-39.

Parent support was measured using the 4- item Autonomy Support Scale developed by Hanna, DiMeglio \& Fortenberry (2005). This scale was designed to 
measure the concept of parental autonomy promoting support in families with an adolescent with T1DM. The scores range from 0 to 32 on the combined frequency and helpfulness of parental support for autonomy scale items. Internal consistency reliability coefficients ranged from $0.77-0.80$ except for a value of 0.67 from the parents' perceived helpfulness version only. Correlations between this scale and several other parental support measures support construct validity (Hanna et al.).

\section{Outcome variables.}

Proximal. SMB were measured by Diabetes Care Activities, a 15-item SMOD-A subscale. This subscale assesses the frequently with which the adolescent performs key activities related to diabetes management (Schilling et al., 2009). Items address specific behaviors associated with diabetes management in an adolescent: testing blood sugar, implementing a meal plan or counting carbohydrates, keeping a record of glucose testing results, taking insulin without reminders, and being prepared for an emergency. Scores can range from $0-45$.

Distal. Outcomes measured included health status indicated by metabolic control and DQOL. Metabolic control was measured by HgbA1c collected at the most recent clinic visit (all reported values were within 1 month of adolescent completing survey). DQOL was measured by the Pediatric Quality of Life Inventory (PedsQL ${ }^{\mathrm{TM}}$ ) 3.0 Type 1 Diabetes Module (Varni, et al., 2003) which addresses problems related to T1DM: physical health (diabetes symptoms, treatment), emotional problems (worry) and communication concerns in youth ages 8-18 years with T1DM. The instrument uses a five point response scale $(0=$ never a problem, $4=$ almost always a problem). A total 
score is recommended and ranges from 0-112. Internal reliability for the total scale score in youth was 0.90 (Varni, Limbers, Burwinkle, Bryant, \& Wilson, 2008).

\section{Data Analysis}

All data were entered into Qualtrics, a Web-based survey software program. Data entered from paper and pencil versions of the survey were double checked for accuracy. Analyses were performed using SPSS (v. 20.0 Chicago, Il., USA) statistical package. A preliminary analysis was conducted to assess for missingness, describe general characteristics of the sample, and estimate the reliability of all research instruments used. Correlational analyses were used to evaluate multicollinearity among the variables of interest. Subscales with reliabilities less than .6 were excluded from the analysis. Relationships between demographic and study variables and relationships within study variables was assessed.

Missing data accounted for less than $5 \%$ of each of the subscales under investigation except for 2 items in the DQOL variable. A majority of survey items had less than 2\% missing data. Little's Missing Completely at Random (MCAR) test was run on variables with missing data. In variables with missing data, Little's MCAR test indicated the findings were not significant. In each case the null hypothesis was rejected, indicating missing data was likely MCAR. Missing data were then imputed using the Maximum Likelihood approach (McKnight et al., 2007).

An analysis of bivariate correlations between all potential predictor and outcome variables was examined (see Table 7). Using the conceptual framework to guide in the selection of key predictor variables, those variables with the highest correlation to one of 
the two outcome variables that could best represent various components of the model were considered for inclusion. Full representation of all the subcategories from each process variable was not possible due to sample size. Two variables from the context dimension (Condition-Specific Factor: complexity of the condition and an Individual Factor: depressive symptoms) and three process variables (Knowledge and Beliefs: selfefficacy, Self-regulation: goals, and Social Facilitation: diabetes communication) with significant correlations $(r=.24-.50)$ to the outcome variables were selected for the hierarchical multiple regressions (HMR) used to analyze the data and test the IFSM model. The goals subscale (a process variable measuring self-regulation) was not significant in any regression models, likely because of the high correlation between goals and communication (.56) and was eliminated in the final model. Power analysis indicated that at least 91 participants were needed to conduct a HMR with 5 variables, assuming a medium effect size, a power of 0.8 and $p$ value of .05 . The distribution of each variable was determined to meet the assumptions required for regression; specifically linearity, normality, and homoscedasticity. Three HMR with variable entry guided by the IFSMT categories were used to address the first two research questions. The first regression tested for context and process variables explaining SMB. Two subsequent regressions tested whether context, process and the proximal outcome variable, SMB was able to predict metabolic control and/or DQOL.

In order to test the third research question addressing whether SM served as a mediator $(\mathrm{M})$ in the model, four conditions are required: It is necessary for the predictor variable $(\mathrm{X})$ (in this case, a process variable) to be associated with the $\mathrm{M}$ (SMB); X must 
be associated with the outcome variable (Y) (metabolic control or DQOL); the M (SMB) must be associated with Y (metabolic control or DQOL), and the strength of the association between $\mathrm{X}$ and $\mathrm{Y}$ must be eliminated or reduced after statistically controlling the M.

\section{Results}

Characteristics of the sample were summarized for all subjects (Tables 5 and 6). The mean age of participants was 14.16 (SD 1.63) reflected a sample representing all study ages but was slightly skewed to the younger side. Adolescents had T1DM for a mean of 5.89 years (SD 3.697); $60 \%$ had lived with T1DM for more than 5 years; $22 \%$ of participants had lived with diabetes for more than 10 years. A majority of adolescents were male (55.2\%). Key findings include: (a) Most adolescents were on an intensive diabetes management regimen with $36.5 \%$ on CSII (infusion pump), (b) Thirty-five percent of adolescents had a T-score greater than 50 on the PROMIS instrument; fifteen percent scored greater than 1SD above the mean and 3\% scored greater than 2SD above the mean, (c) Less than $8 \%$ of subjects experienced a diabetes-related hospital admission in the prior year, (d) Although somewhat difficult to do, parents perceived they were able to manage T1DM, requiring a moderate amount of effort, (e) Three-fourths of the adolescents obtained a score of $\geq 9$ (of 13) on the knowledge survey indicated a relatively good grasp of diabetes SM facts, (f) Adolescents in general had a relatively high level of confidence related to diabetes SM, (g) A majority of adolescents felt they were meeting or had met identified diabetes goals and demonstrated favorable problem solving actions, (h) Communication scores were about the mean, indicating a moderate 
frequency/extent of discussion regarding diabetes related concerns with parents, providers and peers, (i) Generally adolescents perceived parent collaboration regarding SM issues was quite good, (j) Adolescents in the study did not perceive a high degree of parent help or support with regards to deciding on an insulin dose, (k) In general, adolescents performed a moderately high number of SMB activities.

Sizeable correlations included regimen complexity which was moderately associated with metabolic control and in the expected direction. Depressive symptoms were moderately associated with SMB and highly correlated with DQOL. A moderate relationship was found between communication, goal setting and SMB. Self-efficacy, problem solving and communication were moderately correlated with DQOL; SMB was highly correlated with DQOL.

In the first MR analysis (Research Question 1), regimen complexity and depressive symptoms (both context variables) explained $26 \%$ of the variance in the proximal outcome, SMB. After entry of SE and diabetes communication (both process variables) at Block 2, the total additional variance explained by the model as a whole was $12 \%$. In this final model, which explained $37 \%$ of the variance only two variables were significant, depressive symptoms and communication (See Table 8).

Next, a MR analysis was used to evaluate whether select context, process variables and SMB could explain the distal outcomes, metabolic control and/or DQOL (Research Question 2). Regimen complexity and depressive symptoms explained $11 \%$ of the variance in metabolic control. Only regimen complexity was significant at Step 1; using a more complex diabetes regimen explained $11 \%$ of the variance of metabolic 
control. Neither of the regression steps adding process variables or SMB was significant. For DQOL, context (regimen complexity and depressive symptoms) explained $26 \%$ of the variance at Step 1,36\% of the variance when process (self-efficacy and communication) variables were added at Step 2 and $52 \%$ of the total variance was explained when SM behaviors were added at Step 3. In the final model, three variables were significant: depressive symptoms, SE, and SMB (see Table 8). The criteria for mediation (Baron \& Kenny (1986) regarding SMB on process variables was not met (Research Question 3). However, the conditions for mediation were partially met for a context variable; a Sobel test confirmed a (partial) mediation model with depressive symptoms on DQOL once SMB was added (Sobel Test: $-3.45, p<.001$ ).

\section{Discussion}

A prominent finding of the study is the differential role of SMB and the two distal outcomes, metabolic control and DQOL. The patterns of variables related to these two outcomes were also found to be different. In the final regression, depressive symptoms, self-efficacy and SMB were associated with DQOL. With regards to metabolic control, clearly a small but significant portion of metabolic control was explained by regimen complexity. However, a lack of substantive relationships with any of the process variables or SMB is concerning. This is somewhat inconsistent with past studies which found a significant but generally small relationship between process variables and metabolic control. For example, Shorer, David, Shoenberg-Taz, Levavi-Lavi, Phillip, \& Meyerovitch (2011) found an authoritative, low helplessness parenting style was associated with better adolescent diabetes control. Ellis et al. (2004) demonstrated that 
Multisystemic Therapy, a home-based treatment model using family systems theory (cognitive behavioral therapy, parent training) improved metabolic control in teens with poorly controlled T1DM. Wysocki et al. (2007) demonstrated that a 6-month family focused intervention targeting family communication and problem solving positively impacted metabolic control. These differences could be due to the differences in ages of children in the studies, varying measurement of variables of interest such as SM behaviors and use of the child or parent as informant. Clearly further research in this area is needed.

Finding a lack of relationships between any of the process variables and metabolic control may support the assertions of many adolescents and parents that they are doing all they have been advised to do in terms of managing T1DM without being able to significantly impact metabolic control. It appears there remains an unidentified mechanism impacting metabolic control in adolescents with T1DM that has yet to be discovered.

Findings relating to depressive symptoms in this study indicate that although 'subacute' (i.e., not high enough to be classified as “abnormal") were related to health outcomes. Higher levels of depressive symptoms present in adolescents with T1DM can mean fewer SMB and subsequent decreased DQOL. Depressive symptoms have been associated with less frequency of self-blood glucose monitoring (Hilliard, Herzer, Dolan, \& Hood, 2011), poorer metabolic control (Bernstein, Stockwell, Gallagher, Rosenthal, \&Soren, 2013) and an increase in diabetes-related hospitalizations (Stewart, Rao, Emslie, 
Klein, \& White, 2005). The presence of depressive symptoms, even though not extreme, is an important assessment finding in adolescents with T1DM.

In this study, increased SE was associated with increased DQOL. SE theory proposes that confidence in one's ability to engage in a behavior or perform a task determines what behaviors they will participate in, how long they will persist, and the effort they will expend to achieve key goals (Bandura, 1997). As proposed in the IFSMT, engagement in self-regulation behaviors that are supported, leads to engagement in SMB (Ryan \& Sawin, 2009). Further development and study of interventions designed to enhance SE and subsequently SMB is in order.

The role of SMB in this study sheds further light on the importance of this construct. According to IFSMT, adolescents with T1DM will engage in SMB if they have the knowledge and capacity to understanding how to manage T1DM, exhibit selfefficacy, and develop self-regulation skills and abilities to facilitate engagement in SM activities. Based on the exploratory findings from this study, SMB could help us better understand how to improve DQOL in adolescents with T1DM. The analysis also supported the role of direct effects of context and process variables on DQOL which also needs to be further explored.

\section{Limitations}

This study has limitations associated with survey research: A convenience sample was used for this study, thus the research findings are subject to selection bias. An obvious concern regarding a non-experimental design is that MR analysis reveals relationships among variables but does not imply the relationships are causal (Tabachnik 
\& Fidell, 2007). One must be careful not to infer causal relationships between study variables. Another limitation was the validity of data obtained from study participants. There is no way to guarantee that solicited responses were obtained from the intended participant(s) in the study.

The sample was relatively homogenous; participants came from a Midwestern diabetes clinic, the majority of subjects in the study were Caucasian and from 2-parent families and thus the results may not be generalized to other populations. Adolescents with significant depression or depressive symptoms might not be represented in this design. Depressed youth might not keep clinic appointments and therefore would not be appropriately represented in the study sample.

Two instruments lacked optimal reliability: the Condition Management Effort subscale from the Family Management Measure (FaMM) $(\alpha=.57)$; and the SMOD-A Problem Solving subscale (Schilling et al., 2009), $(\alpha=.56)$ (see Tables 5 and 6). Each demonstrated problematic internal consistency reliability leading to the potential for increased measurement variance. Examination of the SMOD-A and FaMM subscales did not reveal items with low item to total correlation. Neither of these subscales has been used widely and may need further testing and possible refinement to substantiate their consistency and accuracy.

\section{Future Research}

It is important to consider what future research directions might be fruitful. Given that data were derived from a cross-sectional sample, the proposed effect of SMB is exploratory at this point. Further longitudinal research is needed to validate what context 
and/or process variables impact SMB and subsequently impact DQOL or metabolic control. There could be value in continuing to study this cohort at predetermined intervals.

The SMOD-A, the first instrument of its kind to measure the SM construct in adolescents has the potential to serve as a valuable tool for measuring distinct components of SM. Further testing/expansion of the instrument is in order to learn how it captures the components of the IFSMT and evaluate the validity and reliability in different samples.

It is possible that SMB mediates the relationship between depressive symptoms and DQOL. A study entertaining a larger sample could help us learn more about how SMB impact this critical context variable along with any potential impact on distal health outcomes.

The relationships within the process components in the correlation matrix (Table 7) raise the possibility of indirect affects that should be explored in future analysis with a larger sample. For example, knowledge, problem solving, goal setting and communication were moderate-strongly related to SE. Similarly, goal setting, autonomy support, and problem solving were related to communication.

Future studies should explore the effects of insulin sensitivity and insulin resistance during adolescence and other biological markers that could help us understand how physiological variables potentially impact health outcomes including metabolic control in adolescents with T1DM. 


\section{Conclusions}

Findings from this study contribute to our understanding of how key context and process variables are related to SM outcomes. Although the current analysis indicates that DQOL can be enhanced by SMB, the same does not hold true for metabolic control. The mechanisms were very different for these two distal outcomes.

The presence of depressive symptoms is a comorbid condition in adolescents with T1DM that can wreak havoc on plans designed to improve SMB and DQOL. In order to impact outcomes in youth with T1DM, it is critical to evaluate mental health status. Further research designed to investigate key variables and relationships within the IFSMT affected by depressive symptoms is needed in order to be able to more fully understand how to optimize SMB. If model assumptions are accurate, enhancing SMB in turn will impact outcomes of adolescents living with this complex chronic disease. 


\section{Reference List}

Amiel, A. A., Sherwin, R. S., Simonson, D. C., Lauritano, A. A., \& Tamborlane, W. V. (1986). Impaired insulin action in puberty: A contributing factor to poor glycemic control in adolescents with diabetes. The New England Journal of Medicine, 315, 215-219.

Baron, R. M., \& Kenny, D. A. (1986). The moderator-mediator variable distinction in social psychological research: Conceptual, strategic, and statistical considerations. Journal of Personality and Social Psychology, 51, 1173-1182.

Bernstein, C. M., Stockwell, M. S., Gallagher, M. P., Rosenthal, S. L., \& Soren, K. (2013). Mental health issues in adolescents and young adults with Type 1 diabetes: prevalence and impact on glycemic control. Clinical Pediatrics, 52(1).

Blethen, S. L., Sargeant, D. T., Whitlow, M. T., \& Santiago, J. V. (1981). Effect of pubertal stage and recent blood glucose control on plasma somatomedin $\mathrm{C}$ in children with insulin-dependent diabetes mellitus. Diabetes, 30, 890-892.

Butler, D. A., Zuehlke, J. B., Tovar, A., Volkening, L. K., Anderson, B., \& Laffel, L. M. B. (2008). The impact of modifiable family factors on glycemic control among youth with type 1 diabetes. Pediatric Diabetes, 9 (Part II), 373-381.

Chui, H.-J. (2005). A test of the Bruhn and Parcel model of health promotion. Journal of Nursing Research, 13(3), 184-196.

DCCT. (1994). Effect of intensive diabetes treatment on the development and progression of long-term complications in adolescents with insulin-dependent 
diabetes mellitus: Diabetes control and complications trial The Journal of Pediatrics, $125,177-188$.

Ellis, D. A., Templin, T., Naar-King, S., Frey, M., Cunningham, P. B., Podolski, C-L., \& Cakan, N.(2007). Multisystemic therapy for adolescents with poorly controlled Type 1 diabetes: Stability of treatment effects in a randomized controlled trial. Journal of Consulting and Clinical Psychology, 75: 168-174.

Grossman, H. Y., Brink, S., \& Hauser, S. T. (1987). Self-efficacy in girls and boys with insulin-dependent diabetes mellitus. Diabetes Care, 10,324-329.

Hanna, K. M., DiMeglio, L. A., \& Fortenberry, J. D. (2005). Parent and adolescent versions of the diabetes-specific parental support for adolescents' autonomy scale: Development and initial testing. Journal of Pediatric Psychology, 30, 257-271.

Hilliard, M. E., Herzer, M., Dolan, L. M., \& Hood, K., K. (2011). Psychological screening in adolescents with Type 1 Diabetes predicts outcomes one year late. Diabetes Research and Clinical Practice, 04(1), 39-44.

Irwin, D. E., Stucky, B., Langer, M. M., Thissen, D., DeWitt, E. M., Lai, J.-S., . . . DeWalt, D. A. (2010). An item response analysis of the pediatric PROMIS anxiety and depressive symptoms scales. Qual Life Research, 19, 595-607.

Jacobson, A. M., Hauser, S. T., Lavori, P., Willett, J. B., Cole, C. F., Wolfsdorf, J. I., .. . Wertlieb, D. (1994). Family environment and glycemic control: A four-year prospective study of children and adolescents with insulin-dependent diabetes mellitus. Psychosomatic Medicine, 56, 401-409. 
Karvonen, M., Viik-Kajander, M., Moltchanova, E., Libman, I., LaPorte, R., Tuomilehto, J., \& al., e. (2000). Incidence of childhood Type 1 diabetes worldwide. Diabetes Care, 23, 1516-1526.

Knafl, K. A., \& Deatrick, J. A. (2003). Further refinement of the family management style framework. J FAM NURS, 9, 232-256.

Knafl, K. A., \& Deitrick, J. (1990). Family management style: Concept analysis and refinement. Journal of Pediatric Nursing, 5, 4-14.

Lewin, A. B., Heidgerken, A. D., Gefken, G. R., Williams, L. B., Storch, E. A., Gelfand, K. M., \& al, e. (2006). The relation between family factors and metabolic control: The role of diabetes adherence. Journal of Pediatric Psychology, 3(2), 174-183.

McKnight, P. E., McKnight, K. M., Sidani, S., \& Figueredo, A. J. (2007). Missing Data: A Gentle Introduction. New York: The Guilford Press.

Moltchanova, E., Schreier, N., Lammi, N., \& Karvonen, M. (2009). Seasonal variation of diagnosis of Type 1 Diabetes in children worldwide. Diabetes Care, 26(7).

Mortensen, H. B., Villumsen, J., Volund, A., Peterson, K. E. \& Nerup, J. (1992). Relationship between insulin injection regimen and metabolic control in young Danish Type 1 diabetic patients. Diabetic Medicine, 9, 834-839.

Murphy, H. R., Wadham, C., Rayman, G., \& Skinner, C. T. (2007). Approaches to integrating paediatric diabetes care and structured education: experiences from the Families, Adolescents, and Children's Teamwork Study (FACTS). Diabetic Medicine, 24, 1261-1268. doi: 10.1111/j.1464-5491.2007.02229.x. 
Olino, T. M., Yu, L., McMakin, D. L., Forbes, E E., Seeley, J. R., Lewinsohn, P. M. \& Pilkonis, P. A.. (2013). Comparison across depression assessment instruments in adolescence and young adulthood: An item response theory study using two linking methods. Journal of Abnormal Psychology (41): 1267-1277.

Ryan, P., \& Sawin, K. J. (2009). The individual and family self-management theory: Background and perspectives on context, process, and outcomes. Nursing Outlook, 57, 217-225.

Schilling, L. S., Dixon, J. K., Knafl, K. A., Lynn, M. R., Murphy, K., Dumser, S., \& Grey, M. (2009). A new self-report measure of self-management of type 1 diabetes for adolescents. Nursing Research, 58, 228-236.

Shorer, M., David, R., Schoenberg-Taz, M., Levavi-Lavi, I., Phillip, M., \& Meyerovitch, J. (2011). Role of parenting style in achieving metabolic control in adolescents with Type 1 diabetes. Diabetes Care, 34, 1735-1737.

Smith, M. J., \& Liehr, P. R. (2003). Middle Range Theory for Nursing . New York: Springer Publishing Company.

Stewart, S. M., Rao, U., Emslie, G. J., Klein, D., \& White, P. C. (2005). Depressive symptoms predict hospitalization for adolescents with Type 1 diabetes mellitus. Pediatrics, 115(5), 1315-1319.

Tabachnick, B. G., \& Fidell, L. S. (2007). Using Multivariate Statistics (Fifth ed ed.). Boston: Pearson.

Varni, J. W., Burwinkle, T. M., Jacobs, J. R., Gottschalk, M., Kaufman, F., \& Jones, K. L. (2003). The PedsQL in Type 1 and Type 2 diabetes: Reliability and validity of 
the Pediatric Quality of Life Inventory generic core scales and type 1 diabetes module. Diabetes Care, 26, 631-637.

Varni, J. W., Limbers, C. A., Burwinkle, T. M., Bryant, W. P., \& Wilson, D. P. (2008). The ePedsQL in type 1 and type 2 diabetes: Feasibility, reliability, and validity of the Pediatric Quality of Life Inventory ${ }^{\mathrm{TM}}$ Diabetes Care 31,672-677.

Whittemore, R., \& Knafl, K. (2005). The integrative review: Updated methodology. Journal of Advanced Nursing, 52(5), 546-553.

Wysocki, T., Harris, M. A., Buckloh, L. M., Mertlich, D., Lochrie, A. S., Mauras, N., \& White, N. H. (2007). Randomized trial of behavioral family systems therapy for diabetes. Diabetes Care, 30, 555-560. 


\section{Chapter 5}

\section{DISCUSSION}

The purpose of this study was to examine self-management (SM) in a cohort of adolescents with T1DM and attempt to identify and further understand factors that contribute to effective SM by testing context, process and outcome variables from the Individual and Family Self-Management Theory (IFSMT) (Ryan \& Sawin, 2009). Results of the study indicated that key context (depressive symptoms) and process (communication) variables were related to SMB. Relationships were found between depressive symptoms (context variable), SE (a process variable), SMB (a proximal outcome) and DQOL (a distal outcome). A prominent finding of this study was the differential role of SMB and the two distal outcomes, metabolic control and DQOL. Although the current study indicated that DQOL can be enhanced by SMB, the same did not hold true for metabolic control. Findings from this study provide nurses responsible for the care of adolescents and their families an understanding of the role SM plays in optimizing quality of life outcomes in adolescents with T1DM. This chapter outlines implications for clinical practice and IFSMT theory, considers a key adolescent mental health policy issue, and concludes with ideas for future research studies.

\section{Clinical Practice}

\section{Meas uring Self-manage ment Variables}

Accurate assessment of SM in adolescents with T1DM is a challenging endeavor limited by the paucity of empirically supported measures. The Self-Management of Type 1 Diabetes Mellitus in Adolescents (SMOD-A) (Schilling et al., 2009), a self-report 
instrument was chosen to measure SM based on its congruence with the definition of SM embodied in the IFSMT and promising psychometric properties. The SMOD-A was designed to measure aspects of diabetes SM in adolescents within 5 subscales: the kinds of collaboration that occurs between a parent and adolescent with T1DM; how often teens perform SM activities and adjust their diabetes regimen; how often parents assist with teens SM needs; the frequency with which adolescents communicates with parents, friends and providers about diabetes-related concerns, and the degree to which diabetes related goals are endorsed by the adolescent. A potential drawback of the SMOD-A is its length: With 52-items it may be too cumbersome to administer in a single sitting, depending on the practice setting. Based on the clinician's focal area of interest, the SMOD-A can readily be adapted to a variety of clinical settings because each subscale can be administered independently (as recommended by the authors). The SMOD-A offers nurses caring for adolescents with T1DM a better understanding of adolescent SMB and the unique processes that contribute to the proximal outcome, SMB.

Another important assessment consideration is adolescent and family knowledge and beliefs. Although not the primary focus, routine assessment of knowledge is useful for developing a baseline, providing the clinician with insight into any potential lack of adolescent/family understanding or misinformation regarding key principles undergirding diabetes SM. All individuals and families need to have at least a rudimentary knowledge of how to manage diabetes at a "survival" level (e.g., know what target blood glucose levels are, what to do with high and low blood glucoses, how to measure and give insulin, understanding how various food affects blood glucoses). Yet there have been 
inconsistent findings in the literature regarding the relationship between knowledge and the impact it has on outcomes. An emerging trend is an emphasis on behavioral strategies to promote effective SM. Piatt (2010) has demonstrated that effective diabetes self-management education combined with behavioral interventions produces better outcomes. In our study, knowledge was moderately correlated with self-efficacy (SE), problem solving, goal setting, and SMB. SE theory proposes that confidence in one's ability to engage in a behavior or perform a task determines what behaviors they will participate in, how long they will sustain a behavior, and the effort they will expend to achieve key goals (Bandura, 1997). As proposed in the IFSMT, "knowledgeable engagement in supported self-regulation behaviors leads to engagement in SMB" (Ryan \& Sawin, 2009, p. 224). Developing interventions to facilitate adolescent SE will likely foster further confidence, independence and higher levels of SM activity.

\section{B uilding Blocks to Diabetes Self-management: Self-Regulation Skills and Abilities}

Nurses play a critical role in helping individuals and families incorporate tasks and skills associated with managing T1DM into everyday life. SMB can be categorized into: (a) essential aspects of TIDM SM; taking insulin, implementing a meal plan, making adjustments in insulin based on results of SMBG; (b) maintaining, obtaining, changing, and creating new meaningful behaviors and (c) dealing with the emotional ups and downs associated with a chronic disease like T1DM (Lorig, 2003). Lorig suggests SM skills encompass problem-solving, decision-making, finding and utilizing resources, forming partnerships with healthcare providers, and taking action. These skills parallel self-regulation skills and abilities and negotiated collaboration found under the umbrella 
of social facilitation in IFSMT. Building diabetes education programs grounded in these principles can help individuals and families successfully weave these tasks and skills into everyday life thus enhancing SMB and quality of life.

Based on National Standards for Diabetes Self-Management Education and Support (Haas, et al., 2014), it is recommended that the adolescent with T1DM and his/her family receive diabetes self-management education (DSME) at the time of diagnosis and at scheduled intervals thereafter. DSME is defined as:

The ongoing processes of facilitating the knowledge, skills, and ability necessary for prediabetes and diabetes self-care. This process incorporates the needs, goals, and life experiences of the person with diabetes and is guided by evidence-based standards. The overall objectives of DSME are to support informed decision making, self-care behaviors, problem solving, and active collaboration with the health care team and to improve clinical outcomes, health status and quality of life. (s145)

Imbedded within this definition are concepts similar to those proposed within the process dimension of the IFSMT; knowledge and beliefs, self-regulation skills and abilities and social facilitation/ negotiated collaboration.

A majority of adolescents participate in survival level DSME aimed at enhancing knowledge and beliefs, self-regulation skills, and social facilitation, yet there are a number of barriers for some other adolescents, such as access, wait times, lack of availability of professionals, and costs associated with educational programming that make attendance difficult. As described in Chapter 2, interventions using computer based Internet platforms are exciting innovations that have the potential to impact access, availability, cost, and clinical outcomes. The framework for this innovation is founded in the science of Interactive Health Communications (IHC). IHC are defined as "the 
interaction of an individual - consumer, patient, caregiver, or professional-with or through an electronic device or communication technology to access or transmit health information or received guidance and support on a health-related issue" (Murray, Burns, Tai, Lai, \& Nazareth, 2005, pg. 2). IHC encompasses technology mediated health communication, excluding direct communication such as face-to-face clinician-patient counseling. Interactive health communications applications (IHCAs) encompass software programs or modules that interface with end-users (Murray et al.) This includes not only health information and support web sites, but clinical decision-support and risk assessment software as well. IHCAs are computer (usually Internet-based) applications with an end-user that combine health information with supports for behavioral change, social support, and/or decision support.

ICSAs have the potential to effectively address many of the diabetes SM needs adolescents and families experience when they attempt to deal with the multitude of issues surrounding how to successfully manage this complex condition. A Cochrane review of 24 randomized studies found Internet-based interventions that combine health information with behavioral change, social, or decision support improved knowledge, perceived social support, behavioral change and clinical outcomes when compared with non-web based control programs (Murray, Burns, Tai, Lai \& Nazareth, 2005). Murray et al. described positive effects on end-users, stating users tend to be "more knowledgeable, feel better socially supported and may have improved behavioral and clinical outcomes compared to non-users" (pg. 2). Benefits associated with IHCAs may include enabling individuals and families to make more informed decisions, promote health behaviors, 
promote peer information exchange and emotional support venues through on-line support communities (Murray et al., 2005). IHCAs are considered superior to the more conventional means of educating individuals and families because even though they serve as a repository for large volumes of data, information can be presented in small "chunks" (Murray et al.). The level of information desired can be tailored to the end-user, and then revisited and shared with others.

\section{B uilding Blocks to Diabetes Self-management: Social Facilitation}

Diabetes education is considered an essential intervention for adolescents and families to actively participate in, yet interventions designed to help the adolescent and family learn survival diabetes SM skills are not adequate to sustain SMB over the long term. To accomplish this, Haas et al. (2014) contend that most individuals and families need ongoing education and support. This concept is also supported in IFSMT; the process dimension encompassing knowledge and beliefs, self-regulation skills and abilities, and social facilitation is foundational to achieving the proximal outcome, SMB.

Diabetes self-management support (DSMS) is defined as those activities that assist the adolescent with T1DM and family members implement and sustain key behaviors required on an on-going basis (Haas et al., 2014). This is an important area for Nursing because of the critical role nurses provide to individuals and families in helping them develop strategies to sustain SMB over time. Nursing support can encompass educational, psychosocial, behavioral and or clinical assistance (Haas et al.). Measures of social facilitation and support related to SMB in this study included autonomy support, adolescent collaboration with parents and providers, and diabetes related communication. 
Social facilitation and negotiated collaboration can further support the processes of informed decision making and problem solving that are ultimately assumed by the adolescent and family.

\section{Variables Impacting Metabolic Control}

An area of clinical interest surrounds whether any of the components from the SMOD-A were correlated with metabolic control in our study. Metabolic control is the most studied outcome measure of SM in adolescents yet the relationship between process variables, SM, and metabolic control remain unclear. Interestingly, diabetes communication was the only process variable to demonstrate any significant relationship with metabolic control in our study $(r=-.23, p<.05)$. Schilling et al. (2009) reported a significant positive relationship between metabolic control (as measured by HgbA1c) and collaboration with parents $(r=.11, p \leq .01)$. The 4 remaining SMOD-A subscales were found to be significant and negatively related to metabolic control ( $r=-.10$ to -.26$)$ in Schilling et al. study, suggesting improved SM is associated with better metabolic control.

Clearly, a small but significant portion of metabolic control was explained by regimen complexity in our study as measured by type of regimen (intensive vs. conventional) and method of insulin delivery (multiple daily injections or continuous subcutaneous insulin infusion (CSII). Ziegler et al. (2011) investigated another component of regimen complexity, frequency of self-monitoring of blood glucose (SMBG) and metabolic control in a large observational cohort $(26,723$ children and adolescents between 0-18 years of age with T1DM). Ziegler et al. found a higher 
frequency of SMBG correlated significantly with better metabolic control with frequency dependent on age and type of regimen: Children under age 6 experienced the highest frequency of SBGM when compared with 6-12 year olds or those older than age 12 (6.0/day vs 5.3 per day vs 4.4 tests per day). Frequency of monitoring was found to be the highest for the continuous subcutaneous insulin infusion (CSII) group when compared with the multiple daily injection group and the conventional therapy group (5.3/day vs 4.7/day vs 4.6/day). On average, one additional SMBG per day resulted in a decrease in $\mathrm{HgbA} 1 \mathrm{c}$ of $0.20 \%$ (Ziegler et al.).

Differences were also found in frequency of monitoring and improved metabolic control between age groups evaluated in the study. Ziegler et al. (2011) suggests that in the adolescent group, testing blood sugars 2-5 times per day significantly improved metabolic control whereas in the two younger groups, increasing the frequency of SMBG beyond two times per day only slightly improved metabolic control. Were other factors working to effect frequency of SMBG and metabolic control? Was the adolescent confident in his/her ability to change their treatment plan? Did they share this information with parents and/or providers and collaboratively make changes to their treatment regimen? Did they change their insulin dose, activity level, or meal plan based on SMBG so as to effect overall metabolic control?

A concerning finding in our study was the lack of any substantive relationships with key process variables and/or the proximal outcome, SMB and metabolic control. Grey, Davidson, Boland and Tamborlane (2001) found consistent parent involvement in daily diabetes management improved metabolic control. Lewin et al. (2006) 
demonstrated a relationship between parental warmth and lower HgbA1c. Murphy, Wadham, Rayman and Skinner (2007) found lower HgbA1c values at one year in adolescents attending four group education sessions grounded in social learning theory. Our study findings suggest that even in the presence of appropriate SMB (thus potentially supporting some adolescents and parents assertions that they are doing all they have been advised to do to effectively manage T1DM), SMB as measured by the SMOD-A did not appear to impact metabolic control in this sample.

\section{Variables Impacting Diabetes Related Quality of Life}

There is no such thing as a day off with T1DM related to the demanding cognitive, behavioral, emotional and social skills necessary to achieve successful outcomes. Attaining optimal DQOL is a challenge for the adolescent, family members and clinicians alike. During adolescence, teens typically take on a greater level of responsibility for managing their diabetes. In our study, DQOL was highly correlated with adolescent depressive symptoms, self-efficacy and SMB.

\section{Nurses Role in Caring for Adoles cents with T1DM}

The prescription for a diabetes treatment regimen is only as sound as the ability and capacity of the adolescent and family to implement it. To better understand how to impact diabetes outcomes, nurses must evaluate key context and process variables in the individual and family unit. This encompasses knowledge and beliefs surrounding various aspects of diabetes SM in the individual and family, the self-regulation skills and abilities essential for SM, and aspects of social facilitation that directly impact SMB in adolescents with T1DM. Family support and involvement in diabetes SM remains a 
crucial element in the equation for achieving optimal diabetes outcomes in the adolescent who must endure the physical and emotional ups and downs of this difficult and often challenging disease. It is imperative for nurses caring for adolescents with T1DM to be capable of evaluating not only the contextual factors and issues surrounding the treatment regimen, but also recognize the importance of process variables that impact SMB and DQOL. Nurses working with challenging cases seek to overcome barriers adolescents and families face in effectively managing this disease or help redefine goals to be more realistic, timely, and attainable. Applying principles from the IFSMT can further this process and ultimately impact SMB and health outcomes.

\section{Health Policy}

\section{Scree ning for Depression in Adoles cents}

Approximately one in five adolescents will suffer an episode of depression by the time they reach adulthood (Bhardwaj \& Goodyer, 2009). Yet a majority of mental illness in adolescents goes unrecognized and untreated during this critical developmental period. Undiagnosed depression can precipitate into significant emotional, social, and academic difficulties during adolescence. Individuals who are ultimately diagnosed and receive treatment for mental illness typically do so only after a long delay, as much as $6-8$ years for those with mood disorders and $9-23$ years for those with anxiety related disorders (Friedman, 2006).

Depression is not easily recognized in adolescents. Rey, Sawyer, Clark and Baghurs (2001) found that parents and teachers were less able to detect internal depressive feelings compared to their ability to note more externalizing behaviors in 
depressed youth. Adolescents are often not skillful at recognizing and seeking help for their depressive symptoms (Friedman, 2006). Therefore, screening for mental health problems in this age group has been advocated by a number of professional medical groups: The American Academy of Pediatrics recommends that questions about depression be asked of adolescents during routine history taking (Foy et al., 2010); the American Medical Association recommends screening adolescents who could be at risk for depression related to family issues, drug or alcohol use, or other indicators of risk (Calonge et al., 2009) and the US Preventive Services Task Force (USPSTF, 2009) recommends screening adolescents age 12-18 years for Major Depressive Disorder (MDD), yet only if systems are in place to assure that an accurate diagnosis can be made and that treatment and follow up mechanisms are available for the adolescents and family.

\section{Barrie rs Associated with Mental Health Screening}

In spite of broad recommendations calling for mental health screening in adolescents there is not unanimous support to do so. Some policymakers believe that universal screening for adolescent depression interferes with parents' rights. They contend that it is the parent(s) who is in the best position to know when their son or daughter is experiencing mental health issues. Therefore, the parent is the best resource for identifying when the adolescent needs assistance to manage a mental health issue. In fact, evidence suggests that parents are often unaware and/or do not recognize the adolescent with suicide ideation or behavior (Joffe, Van Lieshout, Duncan \& Boyle, 2014; Velez \& Cohen, 1988; Walker, Moreau, \& Weissman, 1990). Velez and Cohen 
reported that for a majority of adolescents attempting suicide, there were no warnings given to friends, parents, or siblings either.

There are parents, teachers, and lawmakers who believe that by asking adolescents about depression, mood, or suicide ideation it will create further distress. This is contrary to the findings reported by Gould, Marrocco, and Kleinman et al. (2005) of a randomized controlled trial where adolescents were randomly assigned to either a treatment where they participated in mental health screening with or without questions that probed feelings of suicide and suicide behaviors. They determined there were no differences between groups; those asked probing questions were not any more distressed or suicidal than those who completed the screening without the additional probes (Gould et al.)

A concern has been voiced about the high sensitivity and low specificity of adolescent depression screening instruments because this particular combination leads to more false positive diagnoses. The USPSTF identified two instruments for measuring depression in adolescents exhibiting respectable sensitivity and specificity results when used in primary care settings: Patient Health Questionnaire for Adolescents (PHQ-A) and the Beck Depression Inventory-Primary Care Version (BDI-PC). Sensitivity for the PHQ-A was reported to be $73 \%$ and the BDI-PC, $91 \%$, while the specificity range was 91\% for the BDI-PC and 94\% for the PHQ-A (Calonge, et al, 2009).

Clinical barriers that impede universal mental health screening include lack of time, lack of confidence to treat mental health conditions, long waiting times to see mental health specialists, lack of training and lack of specialists in whom to refer 
adolescents and their family members (Habis, Tall, Smith, \& Guenther, 2007; Horowitz et al., 2002; Olson et al., 2001). Additional barriers for administering screening instruments in an adolescent population found by Taliaferro et al. (2013) included: parent/adolescent concerns about stigma, lack of collaborative care, lack of available resources when suicidality is disclosed by the adolescent, and lack of education materials used to facilitate engagement in the topic of depression with adolescent/parent. Limited clinician opportunities to screen for depression suggest missed opportunities to identify adolescents with mental health issues and missed opportunities to facilitate connecting adolescents and family members to an appropriate mental health service in the course of identifying a problem.

Taliaferro et al. (2013) studied clinician preparation, perceptions, practices, and policies associated with administering a standardized adolescent screening instrument for depression in primary care. Taliaferro et al. (2013) found that $69 \%$ of primary care providers routinely ask about depressive symptoms during health supervision visits with adolescents and about one-half of family medicine physicians, pediatricians, family nurse practitioners and pediatric nurse practitioners reported routinely administering a depression screening instrument. It was noted that primary care providers often use clinical observations and their overall impression to identify adolescents who might be exhibiting signs of depression (Taliaferro et al.).

When surveyed, nurse practitioners in general, felt less prepared to diagnose and treat adolescents with depression than their physician counterparts (Taliaferro et al., 2013). Pediatric clinicians reported greater familiarity with official recommendations 
regarding adolescent depression screening yet this group was less likely (than family providers) to routinely administer standardized depression screening instruments (Taliaferro et al.). A majority of pediatric clinicians only screened high-risk adolescent after they identify warning signs. Approximately $40 \%$ of the primary care providers reported maintaining clear protocols for follow-up after depression screening. Family providers in general were less comfortable than pediatric clinicians in talking about mental health problems.

\section{Depression in Adolescents with Type 1 Diabetes Mellitus}

A variety of factors contribute to the development of depressive symptoms in adolescents. Key established risk factors for depression include a family history significant for parental depression, the experience of a significant major negative life event, a comorbid mental health, or chronic medical condition. Depression is a significant chronic medical problem affecting many adolescents with T1DM. Grey et al. (2002) found the prevalence of depression was 20 percent in youth with T1DM, compared with 7\% for those without diabetes. Grey et al. (1995) indicated that children (age 8 to 14) 2 years post-diagnosis experienced twice the amount of depression than their healthy nondiabetic peers. Hood et al. (2006) found nearly 1 in 7 adolescents with T1DM met the cutoff for depression by self-report and hypothesized that the increased rate of depression is related to the intensification of insulin management that has occurred over the past decade.

Depression contributes to suboptimal T1DM outcomes and as noted above, is relatively common in youth with diabetes. In our study, higher depressive symptoms 
were correlated with less optimal SMB and DQOL. Depression in adolescents with T1DM has been correlated with less frequent SBGM (Hilliard, Herzer, Dolan, \& Hood, 2011), poorer metabolic control (Bernstein, Stockwell, Gallagher, Rosenthal, \& Soren, 2013) and an increase in diabetes-related hospitalizations (Stewart, Rao, Emslie, Klein, \& White, 2005).

Given what we know about the difficulty in recognizing depression in adolescents, the incidence of depression in adolescents, the prevalence of depression in adolescents with T1D and the barriers associated with universal mental health screening, further efforts are necessary to ensure that adolescents are offered and can obtain routine mental health screening for depression and early treatment in a subspecialty setting. An excellent model for developing an approach to a systematic comprehensive depression screening program is described by Corathers et al. (2013).

\section{The Relationships between the States, Medicaid, and Mental Health}

During the most recent recession, state mental health budgets were guttedreductions topped 4.35 billion dollars between FY2009 and FY2012 (Miller et al., 2012). This meant a significant struggle for states trying to meet rising mental health needs with reduced funding resources. In 2013, legislators and governors from many states began the process of restoring state mental health budgets; a majority of states looked to Medicaid for both financing and delivering public mental health services (NAMI, 2013). Medicaid includes provisions for both physical health and mental health care to low income children, pregnant women, families, people age 65 and older and individuals with certain disabilities (Health \& Human Services, 2013). 
States are critical players in the mental health world, historically involved in both establishing and administering a variety of mental health services. Mental health services are often directly funded though state-owned and operated facilities (e.g., community health centers) (NAMI, 2013). States also administer and at least partially fund mental health expenditures through Medicaid programs and often obtain additional federal funds in the form of block grants for the purpose of providing mental health services. Mark et al. (2007) determined that roughly half of all mental health expenditures for mental health services are directly funded or administered by the states.

In 2013, Medicaid financed $27 \%$ of all mental health services in the United States (US) (NAMI, 2013). Medicaid provides access to mental health care for millions of lowincome adults and children in the US that would otherwise be unavailable to them (Health \& Human Services, 2013). Under the Patient Protection and Affordable Care Act (PPACA) Medicaid expanded access to include low income families and children at $138 \%$ of poverty level (Health \& Human Services, 2013). A decision by the Supreme Court in May, 2013 to 'permit' rather than 'require' states to expand Medicaid as part of the PPACA left the resolution to participate in Medicaid in the hands of state legislatures. Currently, 25 states and the District of Columbia are committed to PPACA and the expanded Medicaid programming (NAMI, 2013). Many states cover a broad array of community mental health services and supports through Medicaid programming that is infrequently covered by private insurers. Medicaid services require state Medicaid programs provide physician care, laboratory services, partial hospitalization and Early and Periodic Screening, Diagnosis and Treatment (EPSDT) for youth less than 21 years 
of age (Health \& Human Services, 2013). Under EPSDT, Medicaid eligible youth are entitled to health screening, including mental health screening. The Supreme Court decision directly impacted low-income Americans with mental health needs from states choosing not to participate in the federal Medicaid law.

Before PPACA legislation, families with an adolescent with a pre-existing condition like T1DM were left with few choices for health insurance. They were unable to obtain coverage because they were rejected by the health insurance company based on T1DM as a pre-existing condition or because they could not afford the cost of coverage associated with enrolling in one of the state-run high-risk pools. PPACA included a provision for Americans with pre-existing medical conditions (Health \& Human Services, 2013). Options included finding a state-run insurance plan that could meet their health care needs or enroll in the Federal Pre-Existing Condition Insurance Plan (PCIP). Under PPACA, insurers could no longer discriminate against people with pre-existing health conditions.

\section{Policy Recommendations}

Well child and teen health screenings are established health care practices in the United States. There are adolescent screening guidelines for the progression of normal growth and development, vision, hearing, scoliosis, tuberculosis exposure, and sexually transmitted infections. Stigma, fear, and misinformation should not get in the way of appropriately identifying an adolescent with T1DM with mental health issues, or any adolescent, for that matter who is experiencing depression or depressive symptoms. Universally, all adolescents enrolled in Medicaid should be screened for depression as 
part of the routine preventive care services offered through EPSDT. Comparable universal mental health screening should take place for those selecting insurance from one of the health care Marketplace options. Members of the adolescent care teams responsible for T1DM in adolescents should have routine training in the early warning signs of mental illness in adolescents. Prospective studies should be undertaken to further evaluate cost-benefit analysis associated with early detection and treatment of depression/depressive symptoms in adolescents' vs. delayed diagnosis. In addition, opportunities to study adolescent mental health outcomes in states adopting Medicaid programming and states choosing other methods to manage and fund mental health services for adolescents should be analyzed and compared for cost, quality, and outcomes measures.

\section{Study Limitations}

Study findings must be interpreted in light of several limitations. The sample was relatively homogenous. Participants came from a Midwestern diabetes clinic, a majority of subjects in the study were Caucasian and from 2-parent families. Thus, the results may not be generalized to other populations. Adolescents with significant depression or depressive symptoms might not be represented in this design. Depressed youth might not keep clinic appointments and therefore would not be appropriately represented in the study sample.

An obvious concern regarding a cross-sectional non-experimental design is that regression analysis reveals relationships among variables but does not imply the relationships are causal (Tabachnik \& Fidell, 2007). Because of this, one must be careful 
not to infer causal relationships between the variables. A convenience sample was selected for this study; thus, the research findings are subject to selection bias. Interpreting correlational findings can be difficult given the fact that human behaviors, states, and characteristics are often correlated in complex ways (Polit \& Beck, 2008).

With the focus on the phenomenon of self-management during adolescence, one needs to consider the processes of maturation, physical growth, and emotional maturity occurring during adolescence, potentially rivaling other plausible explanations based on the effects of the predictor variables. The threat of history or external events that take place during the study could affect the criterion variable.

The review of instruments to measure key study concepts was not exhaustive. A potential limitation involves the lack of substantial reliability and validity indicators of select instruments used to measure study variables. Every measurement involves some error that can be minimized by using sound approaches to measurement. Evidence supporting the reliability and validity of the instruments planned for use in this study was acceptable and generally met psychometric standards. The SMOD-A Problem Solving subscale (Schilling et al., 2009) and Condition Management, a subscale from the Family Management Measure (FaMM), (Knafl et al., 2009) demonstrated low internal consistency reliability in our study.

Another limitation involves the validity of the data obtained from study participants. There is no way to guarantee that solicited responses were obtained from the intended participants in the study. The researcher cannot be sure that it was the parent and/or adolescent that completed the appropriate survey or whether the parent- 
adolescent dyad worked together to complete the task thereby potentially affecting the validity of the study findings.

\section{Future Directions for Research}

It is important to consider what future research directions might be fruitful. Given that data were derived from a cross-sectional sample, the analysis conducted to date in no way implies a causal relationship between self-management behaviors and DQOL; instead the proposed effect of SM is exploratory at this point. Further study is needed to validate whether changes in SMB are related to DQOL. MacKinnon (2008) recommends three or more waves of longitudinal data to assess for temporal changes between and over time so as to more accurately validate true mediation effects. Process variables could also possibly mediate context variables; further analysis is needed. The role and impact of parents/family on adolescent SM and ultimately on diabetes outcomes is an important research consideration. Further research is needed on how parents can most optimally be involved in their adolescent's diabetes management in order to facilitate adolescent competence and confidence in managing T1DM. The transfer of diabetes SM responsibilities is a complex process. Process variables related to parent/adolescent relationships and their influence on SMB need to be further examined. To better understand the complexity of this process, longitudinal research studies need to be conducted to better understand the perspective of both the adolescent with T1DM and parent.

The relationships within the process components on the correlation matrix raise the possibility of indirect effects that should be explored in future analysis with a larger 
sample. For example, knowledge, problem solving, goal setting and communication were moderate-strongly related to SE. Similarly, goal setting, autonomy support, problem solving and self-efficacy are related to communication. It is possible that these variables have an indirect impact on SM and DQOL.

Findings from this preliminary work would indicate that there is a need to develop interventions for adolescents with T1DM addressing how to enhance key process variables (SE and self-regulation) in order to foster effective SMB. The SMOD-A, the first instrument of its kind to measure the SM construct in adolescents has the potential to serve as a valuable tool for measuring SM in this population and is one of the first examples demonstrating its usefulness. Further testing of the instrument is in order to learn if it continues to demonstrate acceptable validity and reliability.

When caring for adolescents with T1DM, consideration needs to be given not only to select context variables essential to successfully managing T1DM, but a careful assessment of key process variables as well. As demonstrated in this exploratory study, context and process variables were related to SMB and in turn, SMB played a significant role in DQOL. Further research surrounding IFSMT and its application to adolescents with T1DM and their families is needed.

It was hypothesized that the "honeymoon" or partial remission that some youth with T1DM experience early in the course of the disease characterized by continued endogenous insulin secretion could impact outcomes. Our research findings did not support this. Future studies should explore the effects of insulin sensitivity/resistance and 
glucotoxicity as well as other biophysical markers that might help us better understand how these physiological variables potentially impact health outcomes during adolescence.

Based on the exploratory findings from this study, SMB can play a key role in helping us better understand how to improve DQOL, an important outcome variable in adolescents and families coping with this challenging and complex condition. There is much to learn about how SMB operate to affect health, cost and quality of life outcomes in adolescents with T1DM. Additional studies are needed to help further substantiate the details of these relationships.

\section{Conclusions}

Findings from this study contribute to our understanding of how key context and process variables are related to SM outcomes. Including both context and process variables from the context of adolescents and family members living with a diagnosis of T1DM offers a structure for understanding SMB. The presence of depressive symptoms is a common phenomenon in adolescents with T1DM and often difficult to recognize; even subacute symptoms can have a bearing on SMB. Although the current study indicated that DQOL can be enhanced by SMB, the same did not hold true for metabolic control. Our understanding of how to best impact metabolic control remains elusive. Further exploration is needed in order to better understand what additional factors impact metabolic control in adolescents with T1DM. 


\section{Reference List}

Bandura, A. (1997). Self-Efficacy: The Exercise of Control. New York: W. H., Freeman and Company.

Bernstein, C. M., Stockwell, M. S., Gallagher, M. P., Rosenthal, S. L., \& Soren, K. (2013). Mental health issues in adolescents and young adults with Type 1 diabetes: prevalence and impact on glycemic control. Clinical Pediatrics, 52(1).

Bhardwaj, A., \& Goodyer, I. (2009). Depression and allied illness in children and adolescents: Basic facts. Psychoanalytic Psychotherapy, 23(3), 176-184.

Butler, D. A., Zuehlke, J. B., Tovar, A., Volkening, L. K., Anderson, B., \& Laffel, L. M. B. (2008). The impact of modifiable family factors on glycemic control among youth with type 1 diabetes. Pediatric Diabetes, 9 (Part II), 373-381.

Calonge, N., Tetitti, D., DeWitt, T., \& al., e. (2009). Screening and treatment for major depressive disorder in children and adolescents: U.S. Preventive Services Task Force Recommendation statements Pediatrics, 123, 1223-1228.

Corathers, S. D., Kichler, J., Yayah Jones, N.-H., Houchen, M. J., Morwessel, N., Crawford...Hood, K.K. (2013). Improving depression screening for adolescents with Type 1 Diabetes. Pediatrics, 132 21395-e1402.

Department of Health and Human Services: Center for Medicaid and CHIP Services, Sept 20, 2013. Medicaid and CHIP's New Eligibility Standards. Retrieved from http://www.cms.gov/sitesearch/searchresults.html?q=MAGI\%3A\%20Medicaid\% 
20adn\%20CHIP's\%20New\%20Eligibility\%20Standards\%2C\%20Sept30\%2C\%20 2013

Foy, J., Kelleher, K., \& Laraque, D. for the American Academy of Pediatrics Task Force on Health (2010). Enhancing pediatric mental health care: strategies for preparing a primary care practice. Pediatrics, 125, S87-S108.

Gould, M. S., Marrocco, F. A., Kleinman, M., \& al., e. (2005). Evaluating iatrogenic risk of youth suicide screening programs: A randomized controlled trial. JAMA, 293, 1635-1643.

Grey, M., Cameron, M. E., Lipman, T. H., \& Thurber, F. W. (1995). Psychosocial status of children with diabetes in the first 2 years after diagnosis. Diabetes Care, 18, 1330-1336.

Grey, M., Davidson, M., Boland, E. A., \& Tamborlane, W. V. (2001). Clinical and psychological factors associated with achievement of treatment goals in adolescents with diabetes mellitus. Journal of Adolescent Health, 28, 377-385.

Grey, M., Whittemore, R., \& Tamborlane, W. (2002). Depression in type 1 diabetes in children: Natural history and correlates. Journal of Psychosomatic Research, 53, 907-911.

Grey, M., \& Whittemore, R. T., W. . (2002). Depression in type 1 diabetes in children: Natural history and correlates. Journal of Psychosomatic Research, 53, 907-911.

Haas, L., Maryniuk, M., Beck, C., Cox, C., Duker, P., Edwards, L., . . Kent, D. (2014). National Standards for Diabetes Self-Management Education and Support. Diabetes Care, 37(Supp 1), s144 - s153. 
Habis, A., Tall, L., Smith, J., \& Guenther, E. (2007). Pediatric emergency medicine physicians' current practices and beliefs regarding mental health screening. Pediatric Emergency Care, 23, 387-393.

Hilliard, M. E., Herzer, M., Dolan, L. M., \& Hood, K., K. (2011). Psychological screening in adolescents with Type 1 Diabetes predicts outcomes one year late. Diabetes Research and Clinical Practice, 04(1), 39-44.

Hood, K., K., Huestis, S., Maher, A., Butler, D., Volkening, L., \& Laffel, L. M. B. (2006).Depressive symptoms in children and adolescents with Type 1 Diabetes. Diabetes Care 29, 1389-1391.

Horowitz, S., Kelleher, K., Boyce, T. et al. (2002). Barriers to health care research for children and youth with psychosocial problems. JAMA, 288, 1508 - 1512.

Joffe, B. I., Van Lieshout, R. J., Duncan, L., \& Boyle, M. H. (in press). Suicidal ideation and behavior in adolescents aged 12-16 years: A 17-year follow-up. Suicide and Life Threatening Behavior.

Knafl, K., Deatrick, J. A., Gallo, A., Dixon, J., Grey, M., Knafl, G., \& O'Malley, J. (2009). Assessment of the psychometric properties of the Family Management Measure. Journal of Pediatric Psychology, 1-12. doi: doi:10.1093/jpepsy/jsp034.

Lewin, A. B., Heidgerken, A. D., Gefken, G. R., Williams, L. B., Storch, E. A., Gelfand, K. M., \& al, e. (2006). The relation between family factors and metabolic control: The role of diabetes adherence. Journal of Pediatric Psychology, 3(2), 174-183. 
Lorig, K. R., Sobel, D. S., Ritter, P. L., Laurent, D., \& Hobbs, M. (2003). Selfmanagement and education: History, definition, outcomes, and mechanisms. Annals of Behavioral Medicine, 26, 1-7.

MacKinnon, D. P. (2008). Introduction to statistical mediaton analysis. New York: Lawrence Erlbaum Associates.

Mark, T. L., Levit, K. R., Coffey, R. M., McKusick, D. R., Harwood, H. J., King, E. C., . . . Ryan, K. (2007). National Expendituresfor Mental Health Services and Substance Abuse Treatment, 1993-2003 SAMHSA Publication NO. SMA 07-4227. Rockville, MD.

Murphy, H. R., Wadham, C., Rayman, G., \& Skinner, C. T. (2007). Approaches to integrating paediatric diabetes care and structured education: experiences from the Families, Adolescents, and Children's Teamwork Study (FACTS). Diabetic Medicine, 24, 1261-1268. doi: 10.1111/j.1464-5491.2007.02229.x.

Murray, E., Burns, J., See Tai, S., Lai, R., \& Nazareth, I. (2005). Interactive Health Communication Application for people with chronic disease. Cochrane Database of Systematic Reviews(4). doi: 10.1002/14651858.CD004274.pub4

National Alliance on Mental Illness (2013). Insurance for mental health care: Policy maker's toolkit:Retrieved from: http://www.nami.org/Template.cfm?Section=Health_Care_Reform\&Template=/C ontentManagement/ContentDisplay.cfm\&ContentID =154141.

Olson, A., Kelleher, K., Kemper, K., Zuckerman, B., Hammond, C., \& Dietrich, A. (2001). Primary care pediatricians' roles and perceived responsibilities in the 
identification and management of depression in children and adolescents. Ambulatory Pediatrics, 1, 91-98.

Piatt, G. A., Anderson, R. M., Brooks, M. M., \& al., e. (2010). 3-Year follow-up of clinical and behavioral improvements following a multifaceted diabetes care intervention: results of a randomized controlled trial. . Diabetes Educator, 36, 301-309.

Polit, D. F., \& Beck, C. T. (2008). Nursing Research: Generating and Assessing Evidence for Nursing Practice (Eighth ed.). Philadelphia: Wolters Kluwer, Lippincott, Williams, \& Wilkins.

Rey, J. M., Sawyer, M. G., Clark, J. J., \& Baghurs, P. A. (2001). Depression among adolescents. Medical Journal of Australia, 175, 19-23.

Ryan, P., \& Sawin, K. J. (2009). The individual and family self-management theory: Background and perspectives on context, process, and outcomes. Nursing Outlook, 57, 217-225.

Schilling, L. S., Dixon, J., K., Knafl, K. A., Lynn, M. R., Murphy, K., Dumser, S., \& Grey, M. (2009a). A new self-report measure of self-management of Type 1 diabetes for adolescents. Nursing Research, 58, 228-236.

Schilling, L. S., Dixon, J. K., Knafl, K. A., Lynn, M. R., Murphy, K., Dumser, S., \& Grey, M. (2009b). A new self-report measure of self-management of type 1 diabetes for adolescents. Nursing Research, 37, 87-99. 
Stewart, S. M., Rao, U., Emslie, G. J., Klein, D., \& White, P. C. (2005). Depressive symptoms predict hospitalization for adolescents with Type 1 diabetes mellitus. Pediatrics 115(5), 1315-1319.

Tabachnick, B. G., \& Fidell, L. S. (2007). Using Multivariate Statistics (Fifth ed ed.). Boston: Pearson.

Taliaferro, L. A., Hetler, J., Edwall, G., Wright, C., Edwards, A. R., \& Borowsky, I. W. (2013). Depression screening and management among adolescents in primary care: Factors associated with best practice. Clinical Pediatrics, 52, 557-567.

Velez, C. N., \& Cohen, P. (1988). Suicidal behavior and ideation in a community sample of children: maternal and youth reports. Journal of the American Academy of Child and Adolescent Psychiatry, 27, 349-356.

Walker, M., Moreau, D., Weissman, M. M. (1990). Parents' awareness of children's suicide attempts. American Journal of Psychiatry, 147, 1364-1366

Ziegler, R., Heidtmann, B., Hilgard, D., Hofer, S., Rosenbauer, J., \& Holl, R. (2011). Frequency of SMBG correlates with $\mathrm{HgA} 1 \mathrm{c}$ and acute complications in children and adolescents with type 1 diabetes. Pediatric Diabetes, 12, 11-17. 


\section{FIGURES}

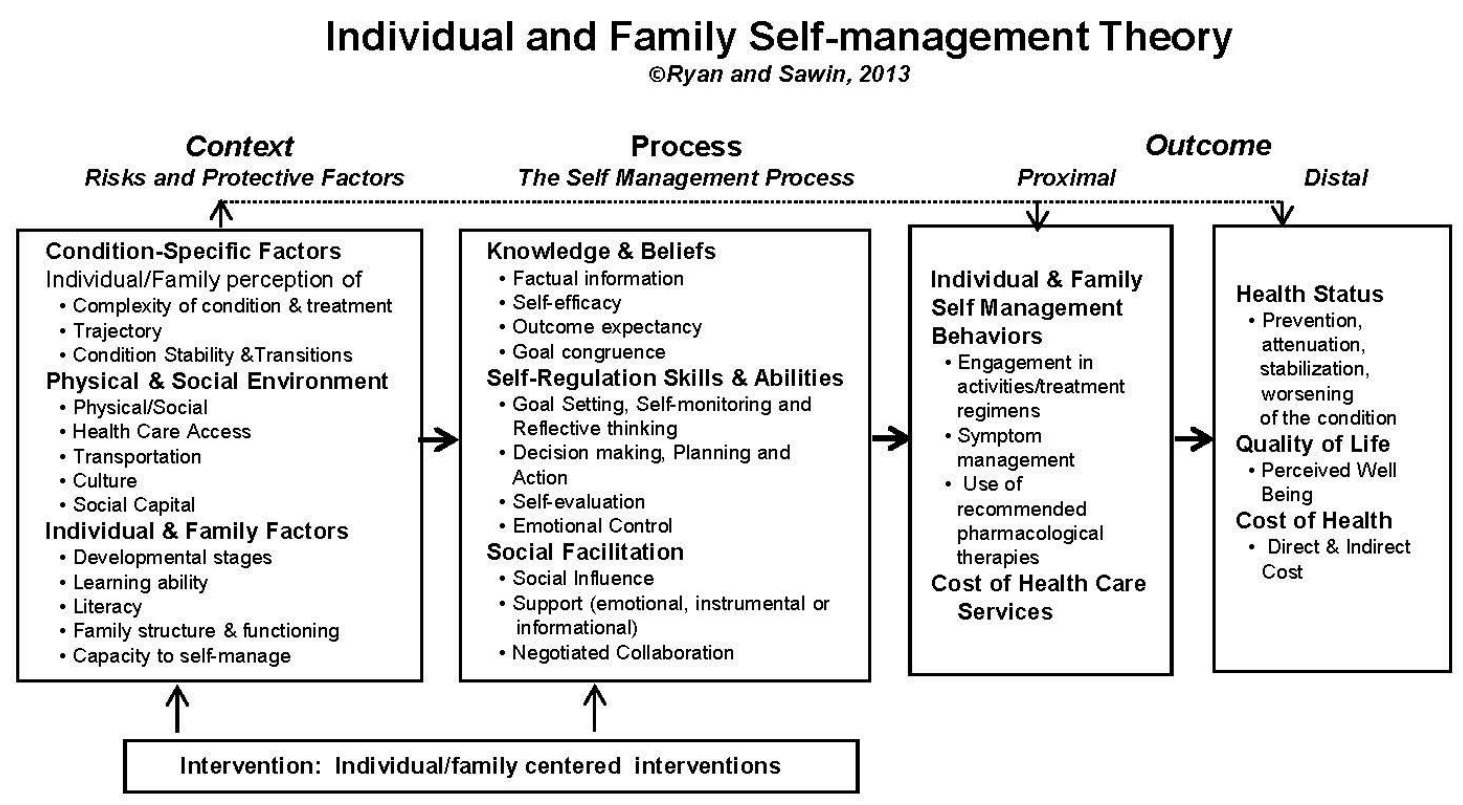

(Self-management Science Center, P20NR0010674)

http://www4.uwm.edu/nursing/about/centers-institutes/self-management.cfm

Ryan, P. \& Sawin, K.J (2009) The Individual and Family Self-Management Theory: Background and Perspectives on Context Process and Outcomes. Nursing Outlook, $57,217-225$.

Ryan, P. (2009). The integrated theory of health behavior change: background and intervention development. Clinical Nurse Specialist, 23(3), 161-170.

Figure 1. UWM Self-Management Science Center Working Group. (2011). Individual and Family Self-management Theory: Definition of self-management. Retrieved from http://www4.uwm.edu/smsc/framework/ 
Behavioral Family Systems Therapy Diabetes (BFST-D) (Wys ocki et al., 2006; Wy socki et al., 2007). Centers on four components: problem-solving, communication, cognitive restructuring and functional-structural family therapy. Sessions are conducted by psychologists or social workers encompassing 12 sessions over a 6 month time frame.

Coping skills training (CST) (Grey et al., 2000). Designed exclusively for adolescents and built into an intensive diabetes management program. CST entails 6 s mall group sessions (problem solving, behavioral modification, and conflict management) conducted by a trainer who role plays various behaviors for dis cussion along with monthly follow-up of youth participating in an intensive diabetes management program. CST goals encompassimproved competence and mastery (and thus more favorable self-efficacy) along with developing more positivestyles of coping related to problems olving, developing social skills, cognitive behavioral interventions and conflict resolution.

Family, Adolescents and Children's Teamwork Study (FACTS) (Murphy, Wadham, Has slerHurst, Rayman, \& Skinner, 2011). A low-intens ity family-centered educational intervention delivered by a multid is ciplinary diabetes teamis designed to take place on the same day as routine clinic visits. The FACTS intervention entails completing 4, hour-long group sessions over a 2 -year period; 2 ses sions are skill based (carbohydrate counting and adjusting ins ulin dos ages) and 2 ses sions encompass interventions based on social learning theory (communication and parental responsibility).

Multisystemic Therapy (MST) (Ellis et al, 2004) Community based, family centered intervention where therapis ts meet with families 2-3 times per week demonstrated a moderately large effect size. Goals are developed at the outset of the program and treatment ceases when goals are met. Mean length of treatment is 6.5 months. Employing cognitive-behavioral therapy, behavioralfamily system therapy and parent training techniques, therapists address problems within the family, peer network, and community (school and extracurricular activities).

"YourWay" (Mulvaney, Rothman, Walls ton, Lybarger, \& Dietrich, 2010). YourWay is website based and depicts barriers and approaches to coping and problem solving through a series of multimedia stories. The siteincludes options for social networkin $\mathrm{g}$, steps to problem

Figure 2. Process-related intervention studies demonstrating greater efficacy. 


\section{Using IFSMT to Optimize the Care of Adolescents with TIDM}

(adapted from (C) Ryan and Sawin, $2013 \mathrm{http} / / \mathrm{www} 4$.uwm edu/nursing/about/centers-institutes/self-management.cfm)

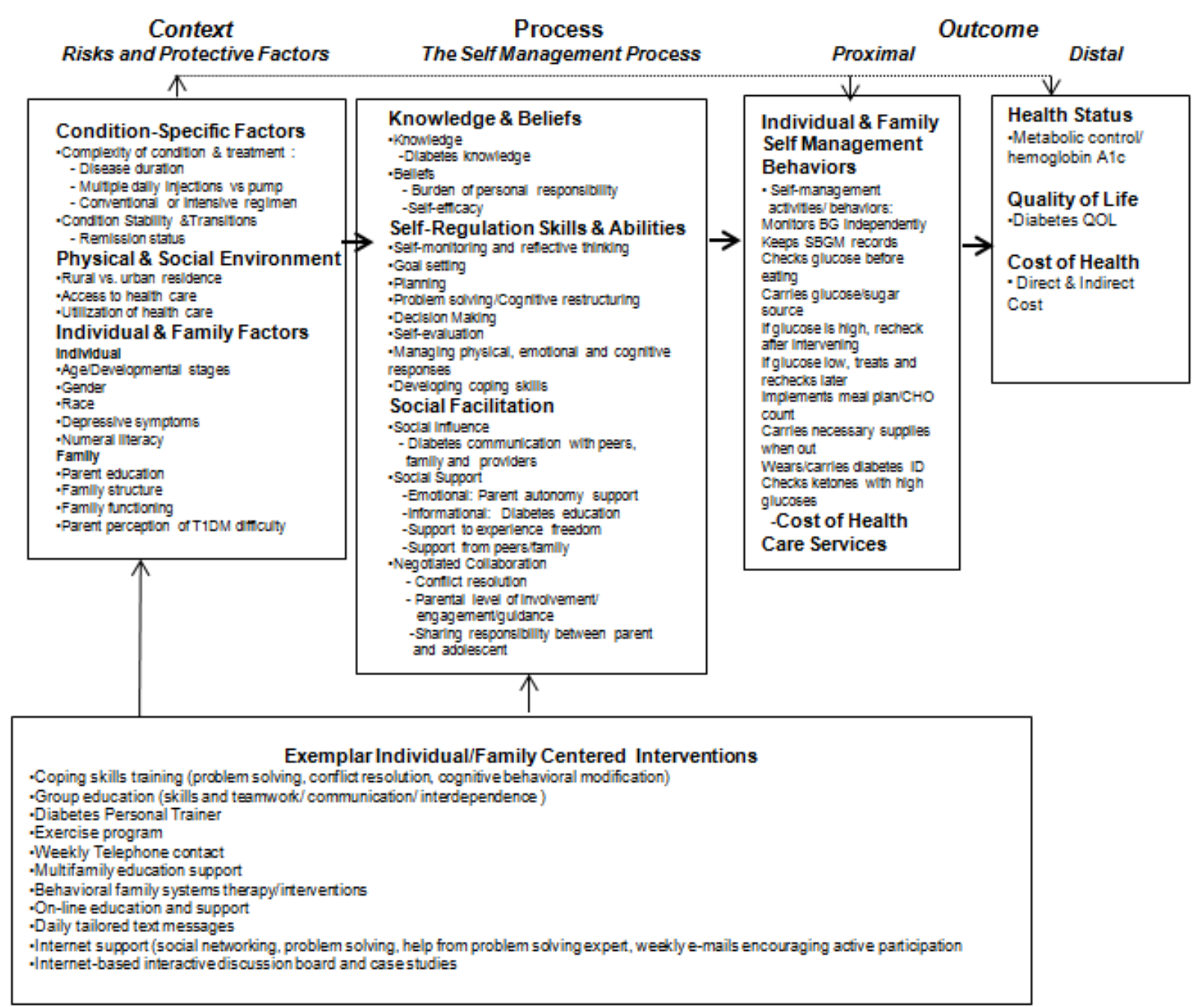

Figure 3. Application of the IFSMT to Care of Adolescents with T1DM. Adapted from:

UWM Self-Management Science Center Working Group. (2011). Individual and Family

Self-management Theory: Definition of self-management. Retrieved from

http://www4.uwm.edu/smsc/framework/ 
MVA VARIABLES=TrQLdm1 TrQLdm2 TrQLdm3 TrQLdm4 TrQLdm5 TrQLdm6 TrQLdm7 TrQLdm8 TrQLdm9 TrQLdm10 TrQLdm11 TrAQLtx1 TrAQLtx2 TrQLtx3 TrQLtx4 TrQLtxll1 TrQLtxl12 TrQLtxl13 TrQLtxl14 TrQLtxll5 TrQLtxll6 TrQLtxl17 TrQLCom1 TrQLCom2 TrQLCom3 /EM(TOLERANCE=0.001 CONVERGENCE=0.0001 ITERATIONS=25).

Figure 4. Syntax from SPSS, Missing Values Analysis, Expectation Maximization for PedsQL data. 
Table 1.

Intervention studies addressing the process dimension of SM.

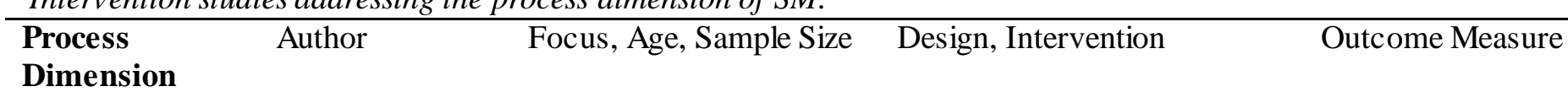

\section{Face to Face/Phone Interventions}

Knowledge \&

Beliefs

Self-Regulation

Skills \&

Abilities

Social

Facilitation

Beliefs

Social

Facilitation
Grey, Boland, Davidson, $\mathrm{Li}, \&$ (coping skills training, Tamborlane (2000) social problem solving, conflict resolution and cognitive behavior modification) combined with intensive diabetes management.

Adolescents only; 12-20 year olds

$\mathrm{N}=77$

Murphy, Wadham,

Rayman, \& Skinner (2007) (FACTS) integrated into
Group education routine care

Teen \& Parent ;12-16 year olds

$\mathrm{N}=78$ families
$\mathrm{RCT}^{\mathrm{D}}$; Random assignment (with or without coping skills training); Coping skills $=6$ small group sessions and monthly follow-up where scenarios focusing on problematic social situations were role played.

$\mathrm{RCT}^{\mathrm{D}}$. Intervention: 4 small group sessions (1 hour each), 2 skills based and 2 based on teamw ork/ communic ation and interdependence.
$6 \mathrm{mo}$. and 1 year post intervention coping skills group had lower HgbAlc values $(t$ $=2.64 *$ and $4.28 *$ respectively); improved medical SE at 6 months $(t=$ $\left.1.98^{*}\right)$ and diabetes $\operatorname{SE}\left(t=2.37^{*}\right)$ at 12 months; and less impact of diabetes on HRQOL $(t=2.07 *$ at 6 months; $t=2.49 *$ at 12 months.

Effect size ${ }^{\mathrm{C}}$

12-month follow up found families attending $\geq 2$ sessions demonstrated increased parental involvement and teens who attended $\geq 2$ sessions had better metabolic control (HgbA1c values decreased by .29\% (vs. increase of .11\% in non-attenders). Difference between HgbAlc of attenders' vs non-attenders was .4\% (effect size: .22).

Effect size ${ }^{\mathrm{A}}$ 


$\begin{array}{lll}\begin{array}{l}\text { Knowledge \& } \\ \text { Beliefs }\end{array} & \begin{array}{l}\text { Murphy, } \\ \text { Wadham, } \\ \text { Hassler-Hurst, }\end{array} & \begin{array}{l}\text { Group education } \\ \text { integrated into routine } \\ \text { care (FACTS) }\end{array} \\ \begin{array}{l}\text { Social } \\ \text { Facilitation }\end{array} & \begin{array}{l}\text { Rayman, and } \\ \text { Skinner (2012) }\end{array} & \text { 12-16 year olds } \\ & & \mathrm{N}=305 \text { teens }\end{array}$

Self-Regulation

Skills \&

Abilities;

Social

Facilitation

Self-Regulation

Skills \&

Abilities;

Social

Facilitation

\section{Nansel et al (2007)}

\section{Cespedes- \\ Knadle \& \\ Munoz (2011)}

Group intervention (activities and skill building workshops) and parallel parent support group

Teens \& Parents

Age $=$ Adolescent

Behavioral self-regulation (Social Cognitive

Theory)

11-16 year old

$\mathrm{N}=81$ teens
$\mathrm{RCT}^{\mathrm{D}}$; Random assignment to intervention vs standard care. Six 90-minute monthly sessions attended by adolescent/ parents encompassing skills training and family teamw ork over 6 months

Teen-Parent dyads; age
$\mathrm{RCT}^{\mathrm{D}}$; Random assignment; randomized within 4 strata: Age (11-13; 14-16) and A1c $(<8$ vs $\geq 8)$ to usual care vs diabetes personal trainer intervention. Personal trainer met with teen-parent dyads and teens alone for total of 6 sessions to assist with selfmonitoring, goal setting and problem solving.

$\mathrm{SGE}^{\mathrm{E}}$. Teen Power is a group intervention for teens \& parents. Groups met weekly (120 minutes) for 10 consecutive weeks. Sessions began with unstructured mealtime. After meal, parent and teen met separately (90 $\mathrm{min}$ ) for process and skills based activities. Treatment terminated when treatment
Primary outcome measure was HgbAlc at 18 months (12 months post intervention): No difference in HgbA1c in either control or study group and no between group differences over time. Poor attendance was major challenge ( $30.4 \%$ attended no sessions; only $48 \%$ of families attended 4 or more education sessions) thus significant missing data. Effect size ${ }^{\text {NA }}$

Trend tow ard intervention effect on A1c found: short term: $F=3.17, P=.06,1$ Year $F=3.79, P=.06$ and significant intervention by age interaction effect for 14-16-year olds only (short term intervention effect $F=4.78, P=.03,1$ Year $F=4.53, P=.04$ )

Effect size ${ }^{\mathrm{NA}}$

Preliminary analysis (still enrolling subjects): data from pre- and posttreatment measures currently being collected indicate "more positive attitude tow ards diabetes among teens"; and decreased caregiver stress.

Effect size ${ }^{\text {NA }}$ 
Self-Regulation

Skills \&

Abilities;

\section{Social \\ Facilitation}

\section{Social}

Facilitation

Lawson et al

(2005)

Social

Facilitation

(2010)

De Wit et al. (2008, 2010)
Faulkner et al.

Personalized exercise intervention

"Sedentary" teens, age 12-19 \& parent

$\mathrm{n}=12 ; 9$ males, 3

females

Frequent, regular telephone contact for teens demonstrating poor metabolic control;

13-17-year olds with A1c $>8.5 \%$ for prior 6 months $\mathrm{N}=46$ goals met.

$\mathrm{SGE}^{\mathrm{E}}$; Voluntary intervention once inclusion criteria met (T1DM, 12-19 years of age; not engaging in regular exercise)

16-week exercise program developed for teens. Parent asked to serve as role model but not required to exercise with teen.

RCT $^{\mathrm{D}}$; Single-blinded, randomly assigned to 6 months of standard DM management or standard care plus weekly telephone contact by Diabetes Nurse Educator (DNE)

$\mathrm{RCT}^{\mathrm{D}} ; 4$ centers randomly determined; 2 centers randomly as signed to HRQOL intervention 2 with standard treatment. Each center set up 3 regular diabetes visits over 12 months; intervention group included assessment of Pediatric QOL Inventory prior to visit; outcomes were discussed face to face during
Teens with > number of episodes of daily exercise (lasting 60 minutes) increased their cardio fitness $\left(r=.59^{*}\right)$. Teens' perceptions of family support for exercise improved after intervention; HgbAlc remained unchanged.

Effect size ${ }^{\mathrm{NA}}$

Regular phone contact for 6 months had no effect on: QOL, family functioning, frequency of BG monitoring, or HgbAlc. Six months post hoc HgbAlc decreased in 6 of 21 in study group ( 0 of 18 in control group)

Effect size ${ }^{\mathrm{N} A}$

Initial results: Intervention group demonstrated more optimal psychosocial well-being, fewer behavioral problems, improved self-esteem/mental health, increased participation in family activities and satisfaction with care (reported effect sizes: .36-.57). Benefits of the intervention disappeared 1 year after intervention terminated. 
in-clinic visit, exploring possible solutions/actions.

Self-Regulation

\section{Skills \&}

Abilities; Social

Facilitation

\section{Social}

Facilitation
Wysocki et al. (2006, 2007)

Family-focused intervention targeting family communication and problem solving

Teen and Parent; 11-16 year olds

\section{$\mathrm{N}=104$}

Ellis et al. (2004, 2007)

Multisystemic Therapy (MST), a home-based
$\mathrm{RCT}^{\mathrm{D}}$; Families randomized to standard care (SC), or SC plus 12 sessions of multifamily educational support (ES) or 12 sessions of Behavioral Family Systems Therapy (BFST) over 6 months. Sessions addressed problem solving, cognitive restructuring, communication, structured family therapy and diabetes specific content. treatment model using social-ecological and family systems theory for poorly controlled T1DM

Mean age, $13.6(\mathrm{SD}=$ 1.6). $\mathrm{n}=25$
$\mathrm{RCT}^{\mathrm{D}}$; Teens randomly assigned to MST or control condition. Targeted high-risk teens. Data collected at

baseline, treatment termination and 6-month post-test. Therapists met with families 2-3 times/wk. for 6 months using MST treatment principles. Family intervention techniques included cognitive behavioral therapy, parent training and behavior family systems therapy, and peer and
Follow up post hoc tests confirmed mean HgbA1c for BFST-D group was lower than SC group at 6, 9, 12, 15, 18 months and the ES group at 9,15, and 18 months Significant group-by-time interaction effect with $F(12,600)=4.29)$ Effect size ${ }^{\mathrm{A}}$

Pilot results: Teens with MST intervention had signific ant improvement in HgbA1c $\left(t=3.33^{*}\right)$ and was clinic ally meaningful: HgbAlc decrease of $2.6 \%$; effect size of .91. Teens demonstrated increased frequency of BG monitoring $(t$ $=-2.80 *$ and decreased number of hospital admissions during the 6-month study period.

Study results: Decrease in Alc in treatment group at treatment termination, effect size $=.41$. At 6 month follow-up, no difference between groups. DKA admissions: A main effect of MST found at treatment termination and follow-up. 
community interventions.

\section{Internet Interventions}

Knowledge \&

Beliefs;

Social Facilitation/

Support

\section{Knowledge \& Beliefs \\ Self-Regulation Skills \& Abilities; Social}

Facilitation

Self-Regulation Skills \& Abilities; Social
Nicholas et al. (2012)

On-line education and peer support

12-17 year olds: $\overline{\mathrm{x}}=$ 14.5

$\mathrm{n}=31$
Franklin,

Waller,

Pagliarit \& Greene (2006)
Daily text messages to a mobile phone designed to reinforce self-management goals based on Social Cognitive Therapy

8-18 year olds.

Mulvaney et al. (2010)
Coping and problem solving based on learning, social-
$\mathrm{NRCT}^{\mathrm{F}}$; Assigned to intervention vs control group; social support measured pre and post-intervention.

8 -week on-line program with 3 components (diabetes related information, interactive learning activities and discussions relevant to teen diabetes-related issues). Eight info modules developed; participant dialogue was asynchronous.

$\mathrm{SGE}^{\mathrm{E}}$; Goal setting in clinic was reinforced by daily text messages from "Sweet Talk" softw are (personalized goalspecific, tailored messages to age, gender, and insulin regimen) for 12 months

$\mathrm{RCT}^{\mathrm{D}}$; Randomized to usual care or usual care plus Internet support. 11 week
Adolescents from 2-parent families in MST group found BGM increased 34\% (effect size: .67) at end of treatment and $27 \%$ (effect size: .53) at 6-month followup. Cost/ participant for MST: \$5-6,000. Effect size ${ }^{\mathrm{C}}$

Qualitative analysis indicates decreased feelings of isolation, know ledge gain, and normalization of experience. Pre-post intervention scores approached significance $(p=.052)$ in one area: "aw areness of relationships with others outside of participants' family" Effect size ${ }^{\mathrm{NA}}$

Intensive therapy + Sw eet Talk improved A1c, self-effic acy and self-reported adherence. $82 \%$ believed Sweet Talk improved diabetes SM; $90 \%$ requested to continue

Effect size ${ }^{\mathrm{NA}}$

Treatment group demonstrated significant improvement (using as-treated analysis) in addressing barriers to $\operatorname{SM}(d=0.64)$; 
Facilitation

Knowledge \&

Beliefs;

Whittemore

et al. (2012)

Self-Regulation cognitive and self-

determination theories

applied to Internet

model

13-17-year olds

$\mathrm{n}=72$

Internet based coping

skills training

(TEENCOPE) vs

Internet education

intervention

(Managing Diabetes).

11-14 year olds

$\mathrm{N}=320$ teens (mean

age: $12.3( \pm 1.1)$ intervention period. Website based interventions involving 6 multimedia stories depicting barriers to SM and approaches to coping/problem solving. Included social netw orking, steps to problem solving, help from problem solving expert, weekly emails encouraging participation.

$\mathrm{RCT}^{\mathrm{D}}$; Randomized to Internet versions of TEENCOPE (with interac tive discussion board) or Managing Diabetes (with interactive case studies, problem solving exercises and tailored responses). Each format included 5 sessions released weekly over 5 weeks. problem solving $(d=.30)$; and Alc $(d=-$ $.28)$ at 12 weeks.

\section{Effect size ${ }^{C}$}

At 6 mo. post intervention, there were no significant between-group treatment effects on primary outcomes (HgbAlc and QOL). Managing Diabetes group demonstrated signific ant increase in social competence over TEEN-COPE group. Effect size ${ }^{\mathrm{NA}}$

\footnotetext{
Note. Effect sizes designated as ${ }^{\mathrm{A}}=$ Small effect $(0.20) ;{ }^{\mathrm{B}}=$ Medium effect $(0.50) ;{ }^{C}=$ Moderate to large effect $(0.60-0.80) ;{ }^{\mathrm{NA}}=\mathrm{Not}$ analyzed.

Designs designated as $\mathrm{RCT}^{\mathrm{D}}=$ Randomized controlled trial; $\mathrm{SGE}^{\mathrm{E}}=$ Single group experimental; $\mathrm{NRCT}^{\mathrm{F}}=\mathrm{Non}_{\text {-randomized }}$ controlled trial;

$* p<.05$
} 
Table 2.

SMOD-A Goals Subscale; EM Coefficients \& Little's MCAR Findings from SPSS Output

\begin{tabular}{|c|c|c|c|c|c|c|c|}
\hline & AG1 & AG2 & AG3 & AG4 & AG5 & AG6 & AG7 \\
\hline AG1 & .566 & & & & & & \\
\hline AG2 & .207 & .378 & & & & & \\
\hline AG3 & .120 & .240 & .503 & & & & \\
\hline AG4 & .153 & .222 & .291 & .428 & & & \\
\hline AG5 & .062 & .082 & .156 & .087 & .533 & & \\
\hline AG6 & .279 & .193 & .129 & .130 & .130 & .522 & \\
\hline AG7 & .255 & .252 & .240 & .154 & .228 & .222 & 677 \\
\hline
\end{tabular}

Table 3.

Comparison of Listwise Deletion, Mean Substitution, and EM Approach using Missing Data from the PedsQL

\begin{tabular}{lccc}
\hline \multicolumn{1}{c}{ PedsQL } & $N$ & Mean & SEM \\
\hline Listwise & 85 & 74.86 & 1.25 \\
Mean Substitution & 104 & 75.05 & 1.13 \\
Mean Substitution (using & 104 & 77.02 & 1.19 \\
PedsQL scoring procedure ) & & & \\
Expectation Maximization & 104 & 77.04 & 1.19 \\
\hline
\end{tabular}


Table 4.

Comparison of Listwise Deletion, Mean Substitution, and Expectation Maximization Approach for Self-Management Activities, Goals, Communication and Depressive Symptoms Instruments

$N \quad$ Mean $\quad$ SEM

SM Activities

Listwise

Mean Substitution

Expectation Maximization
100

104

104
3.16

3.17

3.17
0.04

0.04

0.04

Goals

Listwise

Mean Substitution

Expectation Maximization
101

104

104

95

104

104

Communication
2.77

2.74

2.75

3.41

3.41

3.41
Expectation Maximization

Listwise

Mean Substitution

Depressive Symptoms

Listwise

Mean Substitution

Expectation Maximization
100

104

104
3.16

3.17

3.17
0.05

0.05

0.05
.06

.05

.05 
Table 5. Context Variables $(N=103)$

\begin{tabular}{|c|c|c|c|c|c|c|c|c|c|}
\hline & $N$ & $\%$ & $M$ & $S D$ & Range & $\alpha$ & $\begin{array}{c}25^{\mathrm{th}} \\
\text { percentile }\end{array}$ & $\begin{array}{c}50^{\mathrm{th}} \\
\text { percentile }\end{array}$ & $\begin{array}{c}75^{\mathrm{ln}} \\
\text { percentile }\end{array}$ \\
\hline \multicolumn{10}{|l|}{ Context Variables } \\
\hline Age (years): & & & 14.6 & 1.63 & $12-17$ & & & & \\
\hline 12- year olds & 19 & 18 & & & & & & & \\
\hline 13- year olds & 25 & 24 & & & & & & & \\
\hline 14- year olds & 16 & 15.5 & & & & & & & \\
\hline 15 -year olds & 19 & 18.4 & & & & & & & \\
\hline 16-year olds & 12 & 11.6 & & & & & & & \\
\hline 17-year olds & 12 & 11.6 & & & & & & & \\
\hline Gender: Female & 46 & 45 & & & & & & & \\
\hline \multicolumn{10}{|l|}{ Race } \\
\hline Caucasian & 94 & 91.3 & & & & & & & \\
\hline Black & 6 & 5.8 & & & & & & & \\
\hline Other & 3 & 2.9 & & & & & & & \\
\hline \multicolumn{10}{|l|}{ Fam Structure: Child lives with } \\
\hline Both parents & 89 & 86.4 & & & & & & & \\
\hline Complexity & & & 3.4 & & $2-4$ & & & & \\
\hline 2 & 1 & 1 & & & & & & & \\
\hline 3 & 64 & 62.1 & & & & & & & \\
\hline 4 & 38 & 36.8 & & & & & & & \\
\hline Years with Diabetes & & & 5.89 & 3.69 & $1-13$ & & & & \\
\hline $1-5$ & 53 & 51.4 & & & & & & & \\
\hline$>6$ & 50 & 48.5 & & & & & & & \\
\hline Remission Status & 2 & 1.9 & & & & & & & \\
\hline \multicolumn{10}{|l|}{ Mother's Education } \\
\hline $\begin{array}{l}\text { Attended/Completed high } \\
\text { school }\end{array}$ & 17 & 16.5 & & & & & & & \\
\hline $\begin{array}{l}\text { Attend college or formalized } \\
\text { training }\end{array}$ & 32 & 31.1 & & & & & & & \\
\hline $\begin{array}{l}\text { Complete college or } \\
\text { formalized training }\end{array}$ & 54 & 52.4 & & & & & & & \\
\hline \multicolumn{10}{|l|}{ Father's Education } \\
\hline $\begin{array}{l}\text { Attended/Completed high } \\
\text { school }\end{array}$ & 21 & 20.4 & & & & & & & \\
\hline $\begin{array}{l}\text { Attend college or formalized } \\
\text { training }\end{array}$ & 35 & 33.9 & & & & & & & \\
\hline $\begin{array}{l}\text { Complete college/formal } \\
\text { training }\end{array}$ & 47 & 45.6 & & & & & & & \\
\hline \multicolumn{10}{|l|}{ Residence: } \\
\hline Urban & 83 & 80.6 & & & & & & & \\
\hline $\begin{array}{l}\text { Access to health care (miles from } \\
\text { specialty clinic) }\end{array}$ & & & 33.9 & 24.63 & $1-121$ & & & & \\
\hline Number of clinic visits & & & 4.58 & 1.59 & $2-10$ & & & & \\
\hline $\begin{array}{l}\text { Number of diabetes related } \\
\text { hospitalizations }\end{array}$ & & & 0.12 & 0.44 & $0-3$ & & & & \\
\hline $\begin{array}{l}\text { Depressive Symptoms } \\
\text { FaMM: }\end{array}$ & & & 47.00 & 11.37 & $35-78$ & .95 & 35.00 & 46.00 & 53.00 \\
\hline Life Difficulty ( FLD) & & & 26.90 & 10.26 & $14-58$ & .93 & 17 & 27 & 32 \\
\hline $\begin{array}{l}\text { Condition Mngmt } \\
\text { Ability(CM-A) }\end{array}$ & & & 50.52 & 5.86 & $31-60$ & .81 & 45 & 50 & 54 \\
\hline $\begin{array}{l}\text { Condition Mngmt Effort } \\
\text { (CM-E) }\end{array}$ & & & 13.48 & 3.01 & $6-20$ & .57 & 11 & 13 & 16 \\
\hline
\end{tabular}

Note. ${ }^{a}$ American Indian, Asian, Other. 
Table 6.

Process and Outcome Variables $(N=103)$

\begin{tabular}{|c|c|c|c|c|c|c|c|c|c|}
\hline & $N$ & $\%$ & $M$ & $S D$ & Range & $\alpha$ & $\begin{array}{c}25^{\mathrm{II}} \\
\text { percentile }\end{array}$ & $\begin{array}{c}50^{\mathrm{II}} \\
\text { percentile }\end{array}$ & $\begin{array}{c}75^{\mathrm{In}} \\
\text { percentile }\end{array}$ \\
\hline \multicolumn{10}{|l|}{ Process Variables } \\
\hline Adolescent Knowledge & & & 9.58 & 2.71 & $-13-13$ & & 9 & 10 & 11 \\
\hline Goals subscale ${ }^{\mathrm{b}}$ & & & 16.89 & 3.38 & $6-21$ & .78 & 15 & 17 & 20 \\
\hline Problem Solving subscale ${ }^{\mathrm{b}}$ & & & 13.69 & 2.98 & $5-20$ & .56 & 12 & 14 & 16 \\
\hline $\begin{array}{l}\text { Diabetes Communication } \\
\text { subscale }^{\mathrm{b}}\end{array}$ & & & 15.83 & 4.98 & $5-24$ & .75 & 12 & 16 & 20 \\
\hline Collaboration subscale $^{\mathrm{b}}$ & & & 19.18 & 6.91 & $4-34$ & .82 & 14 & 19 & 24 \\
\hline Self-efficacy $r / t$ diabetes & & & 73.65 & 13.69 & $36-95$ & .86 & 66 & 74 & 85 \\
\hline Autonomy Support Scale & & & 9.15 & 7.23 & $0-24$ & .89 & 3 & 9 & 14 \\
\hline \multicolumn{10}{|l|}{ Outcome Variables } \\
\hline \multicolumn{10}{|l|}{-Proximal outcome } \\
\hline SMB subscale ${ }^{b}$ & & & 30.40 & 5.88 & $15-41$ & .74 & 27 & 31 & 35 \\
\hline \multicolumn{10}{|l|}{-Distal Outcomes } \\
\hline Metabolic & 103 & & 8.66 & 1.43 & $5.8-14$ & & 7.70 & 8.30 & 9.50 \\
\hline Control(HgbA1c) & & 100 & & & & & & & \\
\hline DQOL & & & 51.73 & 10.39 & $28-76$ & .87 & 45 & 52 & 59 \\
\hline
\end{tabular}

Note. ${ }^{b}$ Self-Management of Diabetes in Adolescents (SMOD-A) subscale 
Table 7.

Correlations between Context, Process, and Outcome Variables

\begin{tabular}{|c|c|c|c|c|c|c|c|c|c|c|c|c|c|}
\hline & 1 & 2 & 3 & 4 & 5 & 6 & 7 & 8 & 9 & 10 & 11 & 12 & 13 \\
\hline \multicolumn{14}{|l|}{ Context Variables } \\
\hline 1. Regimen complexity & 1 & & & & & & & & & & & & \\
\hline 2. Age & -.06 & 1 & & & & & & & & & & & \\
\hline 3. Depressive Symptoms & $-.26 * *$ & .12 & 1 & & & & & & & & & & \\
\hline 4. FaMM Life Difficulty & $.22 *$ & -.08 & -.18 & 1 & & & & & & & & & \\
\hline \multicolumn{14}{|l|}{ Process Variables } \\
\hline 5. Adolescent Knowledge & .18 & .15 & -.15 & $.24 *$ & 1 & & & & & & & & \\
\hline 6. Diabetes Self-Efficacy & $.24 *$ & $.25 *$ & -.19 & .14 & $.39 * *$ & 1 & & & & & & & \\
\hline 7. Collaboration w Parents & .04 & $-.39 * *$ & -.14 & -.09 & $-.25 *$ & $-.31 * *$ & 1 & & & & & & \\
\hline 8. Problem Solving & $.24 *$ & $.21 *$ & $-.28 * *$ & .19 & $.28 * *$ & $.59 * *$ & -.08 & 1 & & & & & \\
\hline 9. Diabetes Communicate & $.36^{* *}$ & .03 & -.19 & $.22 *$ & .15 & $.40 * *$ & .19 & $.43 * *$ & 1 & & & & \\
\hline 10. Goal Setting & $.21 *$ & .14 & $-.23 *$ & $.34 * *$ & $.24 *$ & $.51 * *$ & -.07 & $.42 * *$ & $.56 * *$ & 1 & & & \\
\hline 11. Autonomy Support & .16 & -.08 & $-.28 * *$ & -.02 & .10 & .07 & $.47 * *$ & .11 & $.46 * *$ & .18 & 1 & & \\
\hline \multicolumn{14}{|l|}{ Proximal Outcomes } \\
\hline 12. SM behaviors & $.33 * *$ & -.05 & $-.46 * *$ & $.25 * *$ & $.23 *$ & $.24 *$ & $.22 *$ & $.27 * *$ & $.47 * *$ & $.40 * *$ & $.24^{*}$ & 1 & \\
\hline \multicolumn{14}{|l|}{ Distal Outcomes } \\
\hline 13. Metabolic control & $-.32 * *$ & .12 & .17 & -.19 & -.17 & -.14 & -.04 & -.11 & $-.23 *$ & -.17 & -.20 & -.18 & 1 \\
\hline 14. Diabetes QOL & $.22 *$ & .08 & $-.50 * *$ & $.25 *$ & .19 & $.37 * *$ & .05 & $.33 * *$ & $.35 * *$ & $.27 * *$ & $.29 * *$ & $.65^{* *}$ & $-.20 *$ \\
\hline
\end{tabular}

Note. All comparisons were two-tailed and Pearson's $r$ statistics.

* Correlation is significant at the.05 level (2-tailed).

** Correlation is significant at the.01 level (2-tailed). 
Table 8. Hierarchical Multiple Regression Analysis

\begin{tabular}{|c|c|c|c|c|c|}
\hline & $\underline{\mathbf{B}}$ & $\underline{\mathbf{S E}}$ & $\underline{\beta}$ & $\underline{R^{2}}$ & $\underline{\Delta R^{2}}$ \\
\hline Self-Management Behav & & & & & \\
\hline Block 1 & & .37 & & .26 & $.26 * * *$ \\
\hline Regimen Complexity & .19 & & $.23 *$ & & \\
\hline Depressive Symptoms & -.02 & & $-.40 * * *$ & & \\
\hline Block 2 & & .34 & & .37 & $.12 * * *$ \\
\hline Regimen Complexity & .09 & & .10 & & \\
\hline Depressive Symptoms & -.01 & & $-.36 * * *$ & & \\
\hline Diabetes Self-Efficacy & -.00 & & -.01 & & \\
\hline Diabetes Communication & .28 & & $.37 * * *$ & & \\
\hline
\end{tabular}

Metabolic Control $(n=103)$

\begin{tabular}{|c|c|c|c|c|}
\hline Block 1 & & & .11 & $.11 * *$ \\
\hline Regimen Complexity & -.85 & $.30 * * *$ & & \\
\hline Depressive Symptoms & .01 & .09 & & \\
\hline Block 2 & & & .13 & .01 \\
\hline Regimen Complexity & -.73 & $-.26 *$ & & \\
\hline Depressive Symptoms & .01 & .08 & & \\
\hline Diabetes Self-Efficacy & -.08 & -.04 & & \\
\hline Diabetes Communication & -.29 & -.11 & & \\
\hline Block 3 & & & .13 & .00 \\
\hline Regimen Complexity & -.73 & $-.26^{*}$ & & \\
\hline Depressive Symptoms & .01 & .07 & & \\
\hline Diabetes Self-Efficacy & -.08 & -.04 & & \\
\hline Diabetes Communication & -.27 & -.11 & & \\
\hline Self-management Behavior & -.02 & -.01 & & \\
\hline \multicolumn{5}{|l|}{$\operatorname{DQOL}(n=103)$} \\
\hline
\end{tabular}


Regimen Complexity

Depressive Symptoms

Block 2

Regimen Complexity

Depressive Symptoms

Diabetes Self-Efficacy

Diabetes Communication

Block 3

Regimen Complexity

Depressive Symptoms

Diabetes Self-Efficacy

Diabetes Communication

Self-management Behavior
2.33

$-.52$

$-.25$

$-.46$

4.29

4.04

$-1.54$

$-.27$

4.40

$-.13$

14.82
$-.10$

$-.48 * * *$

10.0

.36

$.10 * *$

$-.01$

$-.43 * * *$

$.22 *$

.18

8.69

.52

$.16 * * *$

$-.06$

$-.25 * *$

$.22 * *$

$-.006$

$.51 * * *$ 


\section{SUPPLEMENTAL MATERIALS}

\section{Appendix A: Children's IRB Approval}

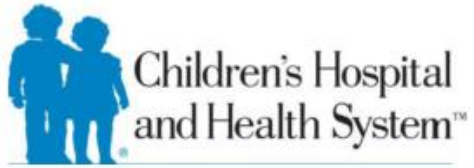

\section{IRB APPROVAL}

October 28,2011

Gwen Verchota, MS, PhD

MS\# 600

Dear Dr. Verchota:

Full Committee approval has been granted on behalf of the Institutional Review Board for your protocol entitled Testing Components of a Self-Management Theory in Adolescents with Type 1 Diabetes Mellitus on September 21, 2011.

For purposes of identification, this research has been assigned the following numbers: CHW 11/164, GC 1405. CHW protocols are also assigned $a$ Grants and Contracts Office [GC] number by the Medical College of Wisconsin.

The Consent, Assent and HIPAA forms approved October 28, 2011 must be used from this date forward.

PLEASE NOTE: PLEASE UN-SHARE THIS PROTOCOL WITH ALL ADMINISTRATIVE PERSONNEL WHO ARE NOT PART OF THE STUDY.

This protocol is approved for 1-year from the original approval date and a continuing review is scheduled for July 19, 2012. Failure to submit the Continuing Review Form in a timely manner may result in the termination of your research approval.

Children's Hospital of Wisconsin and the Medical College of Wisconsin require that all prospectively assigned and intervention or comparison studies be registered according to Public Law 110-85 (also known as the FDA Amendment Act). This was enacted on September 27, 2007 amending the Public Health Service Act to mandate registration and results reporting of applicable clinical trials. Please go to clinicaltrials.gov to register your study.

Any changes in the protocol and any severe untoward reactions must be reported immediately to the Institutional Review Board. Changes in approved research, during the period for which Board approval has already been given, may not be initiated without Board review and approval except where necessary to eliminate apparent, immediate hazards to the human subjects.

When the above work is completed or discontinued, the Board must be notified in order to maintain an accurate record of all current projects. If you leave the community, you are expected to notify the Board to whom the protocol should be transferred; otherwise, the protocol will be terminated. If you have any questions, please feel free to contact the IRB Office at 414-266-7454.

Sincerely,

Signature deleted/recommendation

Robert L. Schum, PhD, Chair

Institutional Review Board $\# 2$

Cc: Kathy Sawin 
APPENDIX B : CONSENT/ASSENT FORM

Children's Hospital and Health System

\section{INSTITUTIONAL REVIEW BOARD \\ STATEMENT OF VOLUNTEER CONSENT FOR RESEARCH STUDY}

TITLE OF STUDY: Testing Components of a Self-Management Theory in Adolescents with Type 1 Diabetes Mellitus

PRINCIPAL INVESTIGATOR: Gwen Verchota, MA, RN, FNP-C, CO-INVESTIGATORS:

Kathleen Sawin, PhD, CPNP-PC, FAAN

Jessica Kichler, PhD

PHONE NUMBER: 1-414-955-3626

FULL STREET ADDRESS: Children's Hospital of Wisconsin, 9000 West Wisconsin Avenue, Wauwatosa, Wisconsin 53226

E-MAIL ADDRESS: ksawin@chw.org

FAX NUMBER: 1-414-266-5731

Complete or attach patient's label to top of consent:

NAME OF SUBJECT: MEDICAL RECORD NUMBER:

We invite you to take part in this research study. Taking part in this research study is your de cision. You do not have to participate. You may stop or decide to leave the study at any time. If you stop or le ave the study, you will not be pe nalized. You will still receive any tre atments, help or benefits coming to you. This form explains what will happen in the research study. The researchers may be re vie wing this form with you and can ans wer any questions you may have. This form also tells you about the risks, discomforts and other information about the study. Medical language is hard to unders tand for most people. If the re is anything that you do not unders tand or are uns ure about, please ask questions. You should only agree to take part in this research study and sign the consent form if you unde rstand what will happen to you, what the risks are, and that your que stions have been answered 


\section{"You" in this consent form means you and/or your child.}

\section{A. WHAT IS THE PROBLEM?}

You are being asked to be in this study because you have Type 1 Diabetes. You are being invited to help us better understand what the things are that are most important to selfmanaging diabetes. With Type 1 Diabetes, you need to be ready and able to make decisions about how much insulin to take and make choices about food intake and exercise many times a day, 365 days a year. Yet we know little about what helps teens learn to make these choices and manage their diabetes and if these self-management activities are related diabetes control and well-being. The information you share will be useful to teens with diabetes and their families as well as health care providers caring for teens with Type 1 Diabetes.

\section{B. WHAT IS THE PURPOSE OF THIS RESEARCH STUDY?}

The purpose of this research study is to learn more about what the things are that can help teens with Type 1 Diabetes and their families manage this disease. We would like to learn whether these things can also affect a teen's overall feeling of well-being. There is a new tool that has been created to measure how teens manage their diabetes and we will be using this new tool in the study.

This research is being done because we do not currently know about all the things that go into how teens and their families manage diabetes on a day-to-day basis. We hope to learn more about many of the things that affect how teens and their families manage Type 1 Diabetes.

Young people 12-17 years of age and a parent are being asked to take part in study. To be in the study you need to have been diagnosed with Type 1 Diabetes for 12 months or longer. Approximately 115 families from Children's Hospital of Wisconsin who have a teen with Type 1 Diabetes will take part in this study.

\section{WHAT IS INVOLVED IN THE RESEARCH STUDY?}

Taking part in this study involves completing a questionnaire and allowing the researchers access to your medical record. You will be asked to complete a questionnaire (there is a different set of questions for the teen and the parent) either on-line or using a paper and pencil version. The questions are about what you do to take care of your health in order to manage your diabetes. We will also be asking you about diabetes, daily activities, feelings about having diabetes, decision making and how your family helps to manage your diabetes. We will also ask about whom you live with, where you reside, and parent(s) level of education. The questionnaire should take each you/your child about 20-30 minutes to fill out. 
Your medical records will be accessed for some key clinical information, specifically, age, weight, when you were diagnosed with diabetes, current insulin dose, type of insulin regimen, whether you use a pump, and the number of times you have been hospitalized because of your diabetes this year.

\section{WHAT ARE THE RISKS TO YOU IN THIS RESEARCH STUDY?}

The questions in the questionnaire are completely voluntary. There are few risks to participating in this questionnaire. We do not expect that completing the questionnaire will be uncomfortable for you, but it may. If you should find filling out the questionnaire makes you uncomfortable, you can skip any questions you wish. You should also feel free to contact the study staff to discuss your discomfort.

There is very small risk of possible loss of confidentiality of your health information. To minimize this risk, your data will be stored either in a locked cabinet and/or in a secure, password protected database with safeguards similar to an electronic health record. Only the approved researchers will have access to your study information.

\section{E. WHAT IF PROB LEMS OCCUR DURING THE STUDY OR WITH TREATMENT?}

If any problems would occur when you and/or your child are filling out the questionnaire you may discontinue at any time. The principle investigator and co-investigators will be available for any concerns. You may withdraw from the study at any time if you change your mind. If you/your child withdraw from the study, the information you completed may be used by the researchers.

One of the things we are looking at in the study is how you are feeling about yourself and your life in general. If we find your score on this part of the questionnaire suggests you have symptoms of depression we would call to talk to you and your parent(s) about this. We would also provide you and your parent with information on who to contact if you wanted to talk more about your feelings.

You/your child do not waive any legal rights by participating in this study or by signing this form.

\section{F. WHAT ARE THE POSSIB LE BENEFITS TO YOU IN THIS RESEARCH STUDY?}

The information we get from you may be useful scientifically and may be helpful to others. We are hopeful this research will teach us more about how teens and families manage Type 1 Diabetes. We can use the information we learn from you to help other teens and families who are dealing with this disease on a day-to-day basis. The questions asked in the interview may produce questions for you/your child to discuss with your health care providers. There may be no direct benefit to you in taking part in this study. 


\section{G. WHAT ARE THE FINANCIAL RISKS TO YOU IN THIS RESEARCH STUDY?}

There are no financial risks for participating in this study.

\section{H. WILL YOU BE PAID FOR TAKING PART IN THE RESEARCH STUDY?}

You and your child will each be given a $\$ 25$ gift card after completion of the questionnaire.

\section{DO YOU HAVE TO PARTICIPATE IN THIS RESEARCH STUDY?}

You do not have to participate in this study. You are free to withdraw at any time. Your decision to withdraw will not change the quality of care that you receive from the health care staff.

\section{J. WHAT IF YOU HAVE MORE QUESTIONS?}

For questions about the study, contact the researchers: Gwen Verchota at 414 -955-3626 or Dr. Kathleen Sawin at 414-955-3612. The research study has also been reviewed and approved by the Institutional Review Board, whose purpose is to see that the rights and welfare of research participants are adequately protected, and that risks are balanced by potential benefits. A member of this committee is available to speak to you if you have any questions or complaints at 414-266-7454.

You will get a copy of this form. A copy of the signed consent, assent (if applicable) and HIPAA Authorization will be kept in your medical record. You may also request a copy of the protocol (full study plan).

\section{K. WILL INFORMATION BE CONFIDENTIAL?}

Children's Hospital of Wisconsin / Children's Health System, its researchers and their designees will maintain the privacy and confidentiality of your personal and health information to the extent permitted by law. Efforts will be made to keep your personal information confidential. We cannot guarantee absolute confidentiality. Your personal information may be disclosed if required by law. Also, scientific data from this study, without identifiable information, may be presented at meetings and published so that it may be useful to others, as long as it is not identifiable with you. An organization that may inspect and/or copy your research records for purposes of quality assurance and data analysis is the Institutional Review Board of Children's Hospital of Wisconsin.

The researcher is required by law to report child abuse or neglect (or suspicion of abuse or neglect) if you mention it to the researcher or if it is suspected. 


\section{PERMISSION TO PROCEED}

The signing of this consent does not release your doctors from their responsibility for your proper medical care at all times.

The proposed research study and consent has been explained to you by:

Name of Principal Investigator or Designee

Signature of Principal or Designee

Date:

When you sign this form, you agree that you have read the above description of this research. You also agree that you have had a chance to discuss the research study with a member of the research team; that your questions have been answered, and that you want to take part in this research.

Signature of Subject or Authorized Representative

Date

Signature of Subject or Authorized Representative

Date

\section{ASSENT OF MINOR:}

The above has been explained to you and you agree to participate.

Signature of Minor

Date

\section{PERMISSION TO CONTACT FOR FOLLOW- UP STUDY}


We may do a follow up study in 1-3 years and would like to contact you about the study at that time. If you were contacted, the study would be explained to you at that time and you would be able to choose whether or not you wanted to be in the study.

Yes, You can contact me for follow up study. You realize that any later study would be explained to you and you could then choose if you wanted to be in the study or not.

No, you would rather not be contacted for a follow-up study.

Signature of Subject or Authorized Representative

Date

Signature of Subject or Authorized Representative

Date 


\section{APPENDIX C: Child Assent Form}

\section{STUDY TITLE: Testing Components of a Self-Management The ory in Adolescents with Type 1 Diabetes Mellitus}

INVESTIGATORS: Gwen Verchota, FNP-C, RN; Kathleen Sawin, PhD, CPNPPC, FAAN, Jessica Kichler, PhD

\section{PHONE NUMBER: 414-955-3612}

NAME OF SUBJECT:

MEDICAL RECORD NUMBER:

We are asking you to be in a research study.

You do not have to be in the study.

If you say "yes" you can quit the study at any time.

Why are you doing the study?

We are doing this study at the Diabetes Clinic at Children's Hospital to learn more about how teens with Type 1 Diabetes take care of themselves and manage their diabetes. We are inviting you to be part of this study because you have Type 1 Diabetes.

What happens if I say yes, I want to be in the study? How long will the study take? Being in the study means answering a set of questions about how you manage your health. There are two ways to answer the questions. You can either ask for a paper copy of the questions that is sent to you and you fill in the answers using a pen or pencil or you can answer the same questions on-line using the Internet.

Examples of questions you will be asked:

- facts about your health,

- what you do to take care of your diabetes,

- how you stay away from problems with your health,

- how your parents helps out with these activities.

We will also ask you about:

- how you make decisions,

- what you do each day to manage your diabetes,

- your feelings.

The study questions you answer either on-line or with paper and pencil take about 20 minutes to complete. We will also get information from your health record at Children's 
Hospital regarding your diabetes (how long you have had diabetes, your weight, insulin dose, and hemoglobin A1c).

\section{What happens if I say no?}

You do not have to be in this study and if you are in it you can stop at any time. If you have any questions please ask the researchers, Gwen Verchota or Dr. Kathy Sawin at 414-955-3612. Do not sign this form if you do not want to be in the study.

\section{Will being in the study help me in any way?}

Being in the study may not help you. Answering the questions about how you care for yourself may bring up questions to share with your parents, doctors or nurses. Mostly the study will help us better understand the kind of things teens do to take care of themselves to manage Type 1 Diabetes that may be useful to other teens, their families and health care providers.

\section{Is there any way the study could be bad for me?}

We do not think that taking part in the study will be uncomfortable for you. If you say you feel sad or upset when you answer the questions, we will discuss this with you and your parent. When you are answering the questions for the study you may choose to skip any question you do not want to answer.

\section{What if problems occur during the study?}

One of the things we are looking at in the study is how you are feeling about yourself and your life in general. If we find you score high on this part of the questionnaire, this would make us concerned that you might be depressed and we would want to talk to both you and your parents about this. We would assist you in finding the kind of help that you need.

\section{Will I get anything for being in this study?}

You will receive a \$25 iTunes gift card as a "thank you gift" after completing the questions in the study. Your parent will also get a \$25 gift card to a local store after he/she completes a set of study questions about having a son or daughter with diabetes.

\section{What happens if I say yes, can I change my mind later?}

If you are in the study you can stop at any time. When you are answering the questions for the study you may choose to skip any question you do not want to answer. If you decide you do not want to be in the study, please tell us.

Who will see my ans wers?

Only the people who work on the study and the people who make sure we run our study the right way will see your answers.

\section{What do I do if I want to be in this study?}


If you want to be in the study sign this form. Your parents / guardian will receive a copy of this form. A copy of the signed consent, assent and HIPAA Authorization will be kept in your medical record.

Writing my name on this page means that the page was read (by me/to me) and that I agree to

be in the study. I know what I will do in the study. If I decide to quit the study, all I have to do is tell the person in charge.

\section{Childs Name}

Child's Signature

Date

Assent Form administered and explained in person by:

Principal Investigator or Designee

Date 
APPENDIX D: Pediatric Endocrinology Section Chief Support for Adolescent Recruitment

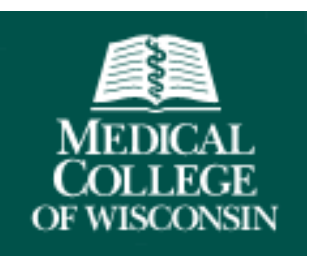

September 21, 2011

Robert Schum, PhD

Chair of the Institutional Review Board

Children's Hospital of Wisconsin

P.O. Box 1997, MS. \#959

9000 W. Wisconsin Ave.

Milwaukee, WI 53226

Dear Dr. Schum:

Dr. Kathy Sawin and Gwen Verchota are planning to conduct a study entitled, "Testing Components of a Self-Management Theory in Adolescents with Type 1 Diabetes Mellitus". The Children's Hospital of Wisconsin Diabetes Clinic will be the only recruiting site for that study. I wholeheartedly support their project, and agree to allow patients to be recruited from the diabetes clinic for their study.

Please feel free to contact me at (414) 266-6750 should you have any questions.

$<$ Signature Removed/Recommendation>

Sincerely, 
Patricia A. Donohoue, M.D.

Professor of Pediatrics

Section Chief

Pediatric Endocrinology and Diabetes 


\section{APPENDIX E: DCIF}

T1 DCIF

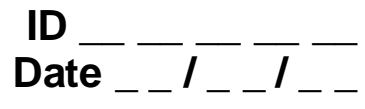

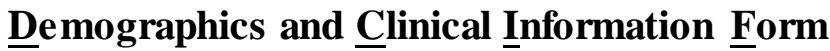

\begin{tabular}{|c|c|}
\hline 1. Date of Birth & Month__ Day _ Year \\
\hline 2. Age & Months \\
\hline 3. Gender & \\
\hline 4. Ethnic Background & $\begin{array}{l}\text { Hispanic/Latino___ / } \\
\text { non-Hispanic/ non-Latino }\end{array}$ \\
\hline 5. Years/months with Type 1 Diabetes & Years_ Months \\
\hline $\begin{array}{l}\text { 6. Complexity } \\
\text { A. Method } \\
1=\mathrm{MDI} \\
2=\mathrm{CSII} \\
\text { B. Regimen } \\
1=\text { Conventional (1 or } 2 \\
\text { injections/day; minimal self- } \\
\text { adjust) } \\
2=\text { Intensive (3+ insulin } \\
\text { injections/day and frequent } \\
\text { adjustments }\end{array}$ & $\begin{array}{l}\text { Method plus regimen = Complexity } \\
\text { Score range: } 2-4 ; \\
\text { total possible score: } 4\end{array}$ \\
\hline $\begin{array}{l}\text { 7. Remission status/Insulin dose- } \\
\text { adjusted HgbA1c (IDAA1C): } \\
\text { A. Hemoglobin A1c } \\
\text { B. Weight } \\
\text { C. Units of insulin in past } 24 \text { hours } \\
\text { Formula: } \\
\text { HgbA1c (percent) }+[4 \text { Xinsulin dose (units of } \\
\text { insulin/kg per } 24 \text { hours] }\end{array}$ & 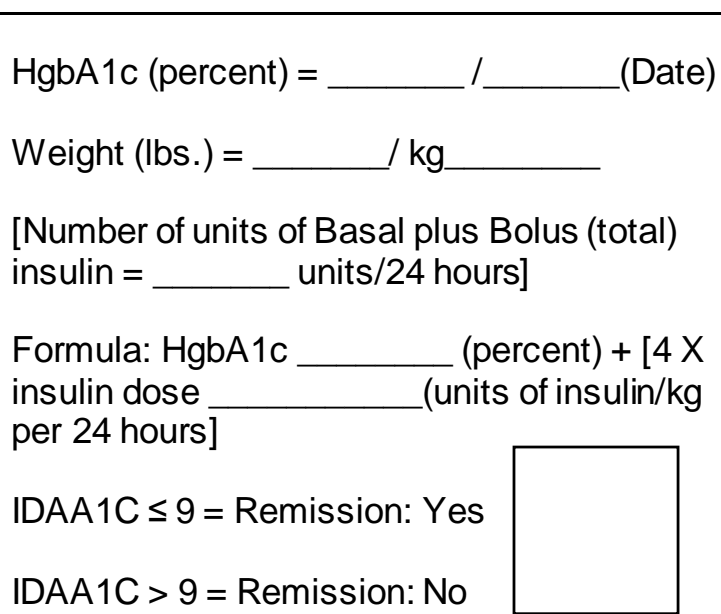 \\
\hline
\end{tabular}




\begin{tabular}{|c|c|}
\hline $\begin{array}{l}\text { 8. Family Structure } \\
-2 \text { parent family = living with } 2 \text { parents } \\
\text { for at least } 50 \% \text { of prior year }=2 \\
-1 \text { parent family = living with } 1 \text { parent } \\
\text { only for at least } 50 \% \text { of prior year }=1 \\
\text {-Teen has not lived with either } \\
\text { biological parent during the preceding } \\
12 \text { months }=0\end{array}$ & $\begin{array}{l}\text { Family Structure (1 [parent], } 2 \text { [both parents], } \\
\text { or } 3 \text { [other arrangement) }\end{array}$ \\
\hline 9. Parents education & 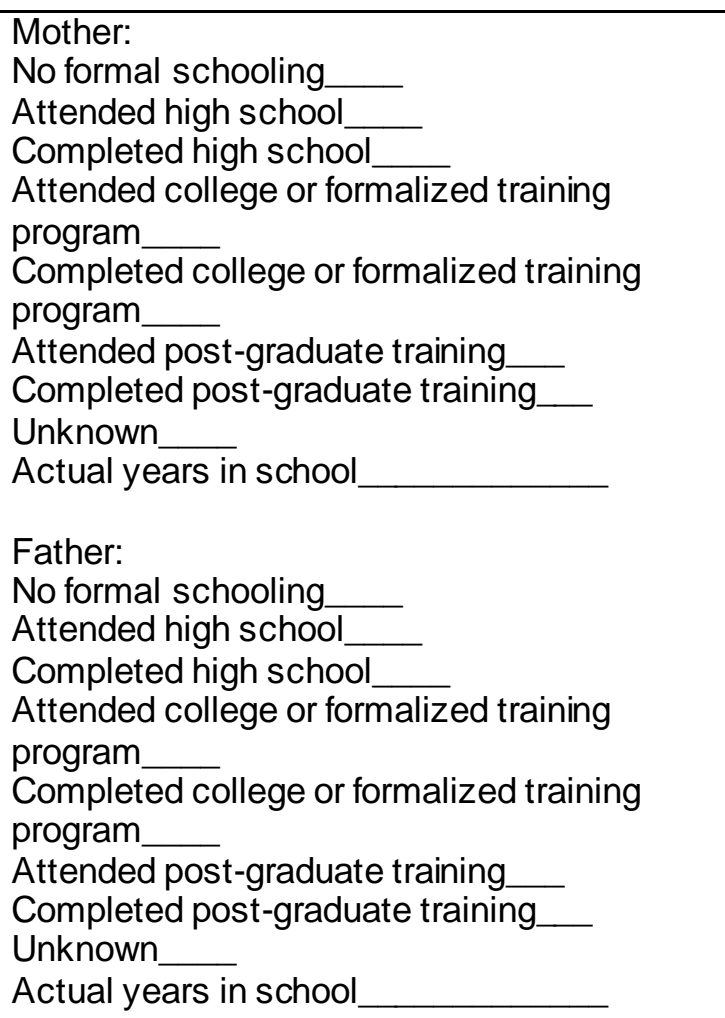 \\
\hline 10. Residence & Address: \\
\hline $\begin{array}{l}\text { 11. Access: Number of miles from home } \\
\text { to Children's Hospital }\end{array}$ & Miles from $\mathrm{CHW}$ to home \\
\hline $\begin{array}{l}\text { 12. Health Care Utilization } \\
\text { A. Number of visits to Diabetes } \\
\text { Clinic/year } \\
\text { B. Number of hospitalization in past } \\
\text { year related to diabetes }\end{array}$ & $A+B$ \\
\hline
\end{tabular}




\section{CURRICULUM VITA \\ Gwen Marie Verchota \\ E-mail: verchota@uwm.edu Linked-In: \\ http://www.linkedin.com/profile/view?id=100693983\&trk=tab_pro}

Education: University of Wisconsin, Doctoral Candidate, $\mathrm{PhD}$ in Nursing

Dissertation: "Testing Components of a Self-Management Theory in Adolescents

with Type 1 Diabetes Mellitus"

University of Minnesota, Family Nurse Practitioner Post-Master's Certificate, College of St. Scholastica, Clinical Nurse Specialist, Adult Medical- Surgical

focus, Masters of Arts, Nursing

College of St. Scholastica, Bachelor of Arts, Nursing

\section{Professional}

His tory:

Care Delivery Manager (2011-present) Responsible for FNP provider hiring, training and care delivery model (direct translational research, protocol development, and best practice design) for virtuwell (www.virtuwell.com), the first on-line convenience care clinic of its kind in the United States.

Family Nurse Practitioner (1998-2011): Provider of independent and collaborative primary, acute, chronic, college health and convenience/urgent care for individuals and families across the lifespan. Developed and implemented primary and tertiary prevention programs (immunization clinics, child and teen check-ups, and women's health care clinics), and a school-based primary care clinic for low income/underserved middle school youth. Mentored NP students from the University of Minnesota, University of North Dakota, South Dakota State University, St. Kate's and the College of St. Scholastica.

Director of Development, 4-year BSN track at Bemidji State University (20062010). Responsibilities included preparation and submission of successful Minnesota Board of Nursing and Commission on Collegiate Nursing Education applications (received 10-year CCNE accreditation for the new program in 2008).

Principal Inves tigator, HRSA Nurse Education, Practice and Retention grant (\$750,000 over 3 years; 2007-2010). Primary author and successful recipient of a MERC/AHEC multidisciplinary practice grant $(\$ 50,000)$, Stratis Palliative Care grant, and several internal education grants (BSU/MnSCU supported funding). Developed curriculum and team taught the following courses in the new BSN program: Nursing Fundamentals, Medical-Surgical nursing, Pediatrics, Health Assessment, and Pathophysiology in both on-line (D2L) and on-campus venues. 
Ass istant/Associate Professor (1991-1996), School of Nursing, College of St. Scholastica. Taught in traditional 4-year BA, Nursing and RN->MA, Nursing programs.

Mate rnal Child Health/Public Health Nursing (1999-2002): Practice and Consultation: Provider of primary and preventive care to low-income, at-risk, and high risk pregnant women, infants, and children in Beltrami County, Minnesota.

Diabetes Educator/Clinical Nurse Specialist: Directed the development of the Duluth Diabetes Center, a regional ambulatory diabetes care center (1983-1991). Responsible for obtaining ADA Program Recognition and directing the care for individuals and families with Type 1, Type 2 and Gestational Diabetes Mellitus.

Staff Regis tered Nurse (1980-1983): Hospital based medical-surgical nursing, Pediatrics and Pediatric Intensive Care.

\section{Select Accomplishments :}

Virtuwell: Translate current scientific evidence into assessment and treatment guidelines for new on-line care delivery platform serving over 100,000 patients with consistent $98 \%$ patient satisfaction ratings.

2013 Tekne Award Recipient for Health Care Innovation of the Year (Company Award)

North Country Health Services: \#1 National Rating, Avatar Patient Satisfaction in Primary Care Provider Survey (2004-2005, 2005-2006).

Pioneer of Advanced Practice role ( $1{ }^{\text {st }}$ FNP to establish a collaborative practice with a Primary Care MD group in rural northern Minnesota community)

AIHA: Member of health care collaborative funded to develop a primary care clinic and patient education/ breast-feeding center in Tomsk, Siberia (partnership program with American International Health Care Alliance, 2002-2006)

Scholarships: Recipient of a Sigma Theta Tau International Theta Xi Chapter Research Scholarship, 2010 Harriet H. Werley Doctoral Student in Research scholarship, and the 2010 Southeastern Wisconsin Research Consortium Scholarship

\section{Licensure and Certification:}

American Nurses Credentialing Center (ANCC), Board Certified as Family Nurse Practitioner (1998-2018)

Minnesota Board of Nursing, Registered Nurse License (089896-6)

Wisconsin Board of Nursing, Registered Nurse and Advanced Nurse Prescriber License (4189-033) 
Prescriptive authority (DEA \#MV2056439)

\section{Organizational Membership}

American Academy of Nurse Practitioners

American Association of Colleges of Nursing

American College of Nurse Practitioners

American Association of Diabetes Educators

American Diabetes Association

Midwest Nursing Research Society

Sigma Theta Tau, International Nurses Honor Society

Society of Adolescent Health

\section{Presentations/Guest Lecturer}

Podcast: "On-line health care: Are We Prepared for this new Innovation?" With Dr. Marti Erickson, PhD, Moderator (momenough.com) April 28, 2014.

Midwest Nursing Research Society, Poster Presentation: Mar 20, 2014. “Testing Components of a Self-Management Theory in Adolescents with Type 1 Diabetes Mellitus"

The Science of Viral and Bacterial Infections: virtuwell Care Providers Series, Health Partners, Oct 2013. Approved for American Nurse Credentialing Center, CME credit.

From Silence to Voice: Our Time Has Come in Professional Nursing. AORN: Minnesota Chapter Meeting, Concordia Language Villages, 9/30/07.

Compassionate Care Giving, $11^{\text {th }}$ Annual Spirituality Conference, Concordia Language Villages, 4/19/2007.

Featured Speaker during National Nurses Week, What Does it Mean to be a Nursing Professional in Health Care Today? 5/12/2007.

How Stella Got Her Groove Back: A Women's Health Symposia Sponsored by North Country Health Services, 2/20/2007.

Consultant for the Innovations in Quality Demonstration Grant for the Minnesota Department of Health: "Adopting a new Continence Program for Residents at North Country Nursing and Rehabilitation Center", April 2007-Dec., 2007. 